\title{
Automated simulations beyond the Standard Model: supersymmetry
}

\author{
Stefano Frixione, ${ }^{a}$ Benjamin Fuks, ${ }^{b, c}$ Valentin Hirschi, ${ }^{d}$ Kentarou Mawatari, ${ }^{e}$ \\ Hua-Sheng Shao, ${ }^{b}$ Marthijn P.A. Sunder ${ }^{f}$ and Marco Zaro ${ }^{g}$ \\ ${ }^{a}$ INFN, Sezione di Genova, \\ Via Dodecaneso 33, I-16146, Genoa, Italy \\ ${ }^{b}$ Laboratoire de Physique Théorique et Hautes Energies (LPTHE), \\ UMR 7589, Sorbonne Université et CNRS, \\ 4 place Jussieu, 75252 Paris Cedex 05, France \\ ${ }^{c}$ Institut Universitaire de France, \\ 103 boulevard Saint-Michel, 75005 Paris, France \\ ${ }^{d}$ Institute for Theoretical Physics, ETH Zürich, 8093 Zürich, Switzerland \\ ${ }^{e}$ Department of Physics, Osaka University, \\ Toyonaka, Osaka 560-0043, Japan \\ ${ }^{f}$ Institut für Theoretische Physik, Westfälische Wilhelms-Universität Münster, \\ Wilhelm-Klemm-Straße 9, D-48149 Münster, Germany \\ ${ }^{g}$ Nikhef, Theory Group, \\ Science Park 105, 1098 XG, Amsterdam, The Netherlands \\ E-mail: stefano.frixione@cern.ch, fuks@lpthe.jussieu.fr, \\ hirschva@itp.phys.ethz.ch, \\ kentarou.mawatari@het.phys.sci.osaka-u.ac.jp, \\ huasheng.shao@lpthe.jussieu.fr, mpasunder@uni-muenster.de, \\ m.zaro@nikhef.nl
}

AbStract: The MadGraph5_aMC@NLO framework aims to automate all types of leading- and next-to-leading-order-accurate simulations for any user-defined model that stems from a renormalisable Lagrangian. In this paper, we present all of the key ingredients of such models in the context of supersymmetric theories. In order to do so, we extend the FEYNRULES package by giving it the possibility of dealing with different renormalisation options that are relevant to supersymmetric models. We also show how to deal with the problem posed by the presence of narrow resonances, thus generalising the so-called on-shell subtraction approaches. We extensively compare our total rate results with those of both Prospino2 and Resummino, and present illustrative applications relevant to the $13 \mathrm{TeV}$ LHC, both at the total-rate and differential levels. The computer programmes that we have used to obtain the predictions presented here are all publicly available.

Keywords: NLO Computations, Supersymmetry Phenomenology

ARXIV EPRINT: 1907.04898 


\section{Contents}

1 Introduction 1

2 Theoretical framework $\quad 4$

2.1 The Minimal Supersymmetric Standard Model 4

2.1.1 Field content and supersymmetric Lagrangian 4

$\begin{array}{lll}\text { 2.1.2 Soft supersymmetry breaking and particle mixings } & 6\end{array}$

2.1.3 Two-component and four-component fermions 8

2.2 Renormalisation 8

2.2.1 Generalities 8

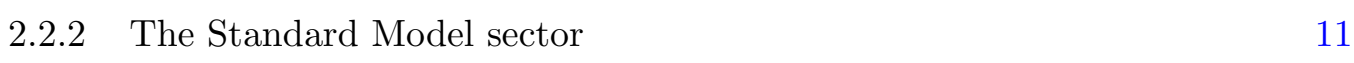

$\begin{array}{lll}2.2 .3 & \text { Gluino renormalisation } & 11\end{array}$

2.2.4 On-shell squark renormalisation 12

\begin{tabular}{ll}
2.2 .5 & Renormalisation of the strong coupling \\
\hline
\end{tabular}

2.3 Technical details on the model implementation in FEYNRULES 16

$\begin{array}{lll}2.4 & \text { Simulations and cross section calculations in SUSY } & 19\end{array}$

3 Validation $\quad 21$

3.1 Setup of the comparison 21

3.2 Degenerate SUSY masses 22

3.3 Non-degenerate SUSY masses 24

$\begin{array}{ll}3.4 & \text { Summary of the comparisons } \\ \end{array}$

4 Total rates for supersymmetric benchmark processes in simplified scenarios

4.1 Setup of the calculation 26

4.2 Production of a pair of SUSY particles of the same species 26

$\begin{array}{ll}4.3 & \text { Production of a pair of SUSY particles of different species }\end{array}$

5 Perturbative computations in the presence of resonances $\quad 32$

$\begin{array}{lll}5.1 & \text { General features } & 32\end{array}$

$\begin{array}{lll}5.2 & \text { Diagram-subtraction procedures } & 37\end{array}$

5.3 Using DR and DS in MG5_AMC 42

6 A case study: jets plus missing energy at the NLO+PS accuracy 43

7 Conclusions $\quad 52$

A Conventions for one-point and two-point functions $\quad 54$ 
B The MoGRE package

B.1 The main method MoGRe\$Renormalize and its options 55

$\begin{array}{lll}\text { B.2 Simplifying the procedure } & 56\end{array}$

$\begin{array}{lll}\text { B.3 Restrictions } & 58\end{array}$

$\begin{array}{lll}\text { B.4 Specifying the renormalisation scheme } & 58\end{array}$

$\begin{array}{ll}\text { B.5 Clearing a renormalisation scheme } & 59\end{array}$

B.6 Technical details on the functioning of the method 59

$\begin{array}{ll}\text { B.6.1 Initialization } & 59\end{array}$

$\begin{array}{lll}\text { B.6.2 Field renormalisation } & 60\end{array}$

$\begin{array}{lll}\text { B.6.3 Parameter renormalisation } & 61\end{array}$

B.6.4 Renormalisation of the Lagrangian 61

C Decoupling of heavy SUSY particles in MG5_AMC 62

\section{Introduction}

After more than fifty years since its proposal, the Standard Model (SM) has been proven to be an extremely successful theory of Nature: its predictions agree well with the vast majority of the data collected so far, sometimes at an astonishing level of precision. Despite its success, however, the SM leaves some deep questions unanswered, and suffers from various conceptual issues and limitations; thus, it is widely understood as a low-energy effective theory that is supposed to emerge from a suitable UV-complete theory. Among the candidates for the latter, weak-scale supersymmetry (SUSY) constitutes one of the best-motivated options from a theoretical viewpoint. Naturally extending the Poincaré algebra by linking the fermionic and bosonic degrees of freedom of the theory [1-9], SUSY and in particular its minimal incarnation, the so-called Minimal Supersymmetric Standard Model (MSSM) [10, 11], addresses several of the shortcomings of the SM. For example, SUSY can tackle some aspects of the hierarchy problem inherent to the SM by stabilising all scalar masses relatively to quantum corrections [12], it can ensure the unification of the three SM gauge couplings at high energies [13-15], and many SUSY realisations include a candidate explaining the presence of dark matter in the Universe [16, 17].

The existence of a superpartner with a mass equal to the one of its SM counterpart is experimentally excluded, so SUSY must be broken. For theoretical and phenomenological reasons, this breaking must be soft and is expected to shift the SUSY particle masses in the $\mathrm{TeV}$ regime. Thus, also owing to the solid theoretical motivations of supersymmetric theories, the quest for SUSY particles still plays a major role in the searches carried out by the LHC experiments, and features prominently in the strategies that are currently being laid out for future-collider projects. Both the ATLAS and CMS collaborations have begun to release full LHC run 2 results; the persistent absence of beyond-the-SM (BSM) signals implies that the space of parameters of SUSY theories is constrained in an increasingly severe manner. For example, squarks and gluinos are bounded to have masses well above 
1-2 TeV [18-21], whilst the bounds on the electroweak superpartners now reach several hundreds of $\mathrm{GeV}[22,23]$. However, such experimental limits have been extracted either in the framework of specific MSSM benchmark scenarios, or in the context of simplified models of new physics that are inspired by the MSSM, and that feature only a small number of new particles and new interactions with respect to those of the SM [24, 25]. Therefore, these limits can be evaded both in various non-minimal supersymmetric theories and with specific, fine-tuned, parameter configurations of the MSSM.

Experimental analyses for SUSY searches are currently mostly based on Monte Carlo simulations of signals in which tree-level matrix elements of different partonic multiplicities are consistently combined and interfaced with parton-shower (PS) Monte Carlos; the absence of double counting between matrix elements and parton showers is guaranteed by the use of a merging prescription - CKKW [26, 27], MLM [28], and CKKW-L [29, 30] (see also refs. [31-33]). For any given jet multiplicity, such predictions are therefore leadingorder (LO) plus leading-logarithm (LL) accurate. They are typically further improved by normalising them, at the fully inclusive level, to the best available (in the sense of perturbative information) total cross sections for the production of the relevant SUSY particles. Typically, these cross sections combine fixed-order predictions at the next-to-leading-order (NLO) accuracy in $\alpha_{S}$ (which we shall call "NLO QCD" henceforth, understanding that quarks, gluons, squarks, and gluinos run in the loops), with calculations that carry out the resummation of threshold logarithms at the next-to-leading-logarithmic (NLL) accuracy. In some cases, the results include some of the next-to-next-to-leading-order (NNLO) and next-to-next-to-leading-logarithmic (NNLL) contributions as well.

As the LHC moves steadily towards a precision-physics phase, BSM search strategies, in view of their null results thus far, must evolve too. Among other things, finding elusive signals requires improving the control over systematics. At the theoretical level, this demands a better use of higher-order results than the simple rescaling of tree-level merged simulations, without losing the realistic description of multi-jet final states provided by parton showers. Fortunately, solutions originally devised for SM physics, which have been thoroughly and successfully tested since the start of the LHC operations, can be applied to BSM scenarios as well, pretty much as their tree-level counterparts. More specifically, NLO computations can be matched to parton showers (NLO+PS henceforth) by means of the MC@NLO [34] and Powheg [35] methods (or of any of their variants and less-used alternatives [36-43]) for any underlying SUSY process. Likewise, the extensions of treelevel merging techniques to NLO [44-55] work equally well in the SM and BSM theories. These facts have stimulated recent theoretical work (which we shall briefly review in section 2.4), that is characterised by being essentially on a process-by-process basis and in simplified models.

The main goal of this paper is to render systematically feasible, for any user-defined process and in the fully-fledged MSSM, fixed-order, matched, and merged simulations that include NLO QCD effects. In order to do this, we shall rely on automated techniques, which are by now extremely well established; in particular, we shall work in the MADGrAPh5_aMC@NLO framework [56] (MG5_AMC henceforth). We remind the reader that MG5_AMC is a meta-code, which uses hard-wired information on Quantum Field Theories 
(such as general rules of Feynman diagrammatics, the structures of matrix elements, phase spaces, and cross sections, and so forth), and external information equivalent to the Lagrangian of the theory one is interested in (these are a set of rules, called a UFO model [57], obtained automatically from the Lagrangian by means of codes such as FeynRules [58], possibly used jointly with NLOCT [59]), to construct on the fly a computer code specific to the production process that one is interested to simulate.

Thanks to the significant amount of development and validation activity performed on MG5_AMC in the past few years, most of the work for the current paper has gone into the construction of a UFO model for the MSSM (see section 2). A notable exception, at the level of the meta-code, is the following. In the context of theories with rich particle spectra, that include relatively narrow resonances, the computation of contributions beyond the LO might give results apparently preventing the series from being well-behaved. This happens when real-emission corrections include partonic sub-processes that feature those resonances in $s$-channels, whose integration over the phase space is either divergent (if the resonance propagator is not Dyson-summed), or grows like an inverse power of the resonance width (and is therefore numerically dominant). A familiar example in the SM is that of $t W^{-}$ production, for which a subset of real corrections features a $t W^{-} \bar{b}$ final state, to which diagrams with an $s$-channel $\bar{t}$ quark contribute. We point out that the lack of convergence of the perturbative series in these cases is perfectly justified, since one is trying to compute higher-order corrections to the cross section of a process which is simply ill-defined. In the SM example just mentioned, $t W^{-}$production does not exist as such: its definition requires the simultaneous presence of a particle (the top) and of one of the decay products of its antiparticle (the $W^{-}$). In spite of this, one can try and give an operative meaning to these ill-defined processes: they are conceptually useful, since they correspond to an intuitive physical picture which is easy to understand, and they can constitute, within reasonable approximations, valuable perturbative tools. In this sense, BSM theories in general, and SUSY in particular, provide one with many possibilities to test different strategies. In this paper we shall discuss the definitions of such strategies, that we collectively call Simplified Treatments of Resonances (STR henceforth), and their implementations in MG5_AMC in a way suited to NLO+PS simulations. Thus, STR encompass the procedures known as Diagram Removal (DR) and Diagram Subtraction (DS), and generalise the so-called onshell subtractions schemes.

This paper is organised as follows. In section 2 we briefly review the status of simulations in SUSY theories, and the basic characteristics of MG5_AMC and of the models it uses for BSM physics. We also introduce the theoretical framework of the MSSM, and its various renormalisation options. We validate our implementation against PROSPINO2 and Resummino in section 3. The total cross sections of several SUSY benchmark processes in simplified scenarios are presented in section 4 . The general algorithms for the treatments of resonances in perturbative computations, and their implementations in MG5_AMC, are discussed in section 5. A case study of jets plus missing energy at NLO+PS accuracy at the $13 \mathrm{TeV}$ LHC is considered in section 6, in the context of a non-simplified benchmark point. We draw our conclusions in section 7. Some details about one- and two-point loop integrals, the MoGRE package (the acronym standing for More General $\underline{\text { Renormalisation }}$ 
in FeynRules), and the settings relevant to the decoupling mass limit in MG5_AMC can be found in the appendices $\mathrm{A}, \mathrm{B}$, and $\mathrm{C}$, respectively.

\section{Theoretical framework}

\subsection{The Minimal Supersymmetric Standard Model}

\subsubsection{Field content and supersymmetric Lagrangian}

The MSSM, the simplest SUSY extension of the SM, results from the direct supersymmetrisation of the SM [10,11]. The gauge symmetry group of the theory is the SM one, and it relies on the three vector supermultiplets $V_{B}, V_{W}$ and $V_{G}$,

$$
V_{B} \equiv\left(B_{\mu}, \widetilde{B}^{\alpha}\right), \quad V_{W} \equiv\left(W_{\mu}, \widetilde{W}^{\alpha}\right), \quad V_{G} \equiv\left(G_{\mu}, \widetilde{G}^{\alpha}\right),
$$

that are respectively connected to the hypercharge $\mathrm{U}(1)_{Y}$, weak $\mathrm{SU}(2)_{L}$ and strong $\mathrm{SU}(3)_{c}$ symmetries. All the SM gauge bosons $B_{\mu}, W_{\mu}$ and $G_{\mu}$ are supplemented by their fermionic gaugino superpartner $\widetilde{B}, \widetilde{W}$ and $\widetilde{G}$, all fields lying in the adjoint representation of their respective gauge group. The matter sector of the theory includes three generations of five chiral supermultiplets $L_{L}, E_{R}, Q_{L}, U_{R}$ and $D_{R}$,

$$
\begin{array}{rlrl}
L_{L}(\mathbf{1}, \mathbf{2},-1 / 2) & \equiv\left(\ell_{L}=\left(\begin{array}{c}
\nu_{L} \\
e_{L}
\end{array}\right), \widetilde{\ell}_{L}=\left(\begin{array}{c}
\widetilde{\nu}_{L} \\
\widetilde{e}_{L}
\end{array}\right)\right), & & E_{R}(\mathbf{1}, \mathbf{1}, 1)=\left(e_{R}^{c}, \widetilde{e}_{R}^{\dagger}\right), \\
Q_{L}(\mathbf{3}, \mathbf{2}, 1 / 6) & \equiv\left(q_{L}=\left(\begin{array}{c}
u_{L} \\
d_{L}
\end{array}\right), \widetilde{q}_{L}=\left(\begin{array}{c}
\widetilde{u}_{L} \\
\widetilde{d}_{L}
\end{array}\right)\right), \\
U_{R}(\overline{\mathbf{3}}, \mathbf{1},-2 / 3) & =\left(u_{R}^{c}, \widetilde{u}_{R}^{\dagger}\right), & D_{R}(\overline{\mathbf{3}}, \mathbf{1}, 1 / 3)=\left(d_{R}^{c}, \widetilde{d}_{R}^{\dagger}\right),
\end{array}
$$

where the SM left-handed $\left(q_{L}\right)$ and right-handed $\left(u_{R}\right.$ and $\left.d_{R}\right)$ quarks, as well as left-handed $\left(\ell_{L}\right)$ and right-handed $\left(e_{R}\right)$ leptons, are complemented by scalar counterparts, the lefthanded $\left(\widetilde{q}_{L}\right)$ and right-handed $\left(\widetilde{u}_{R}\right.$ and $\left.\widetilde{d}_{R}\right)$ squarks and left-handed $\left(\widetilde{\ell}_{L}\right)$ and right-handed $\left(\widetilde{e}_{R}\right)$ sleptons. We have moreover indicated, in the equation above, the representation of the various supermultiplets under the MSSM gauge group $\mathrm{SU}(3)_{c} \times \mathrm{SU}(2)_{L} \times \mathrm{U}(1)_{Y}$. The MSSM Higgs sector is constituted of two chiral supermultiplets $H_{D}$ and $H_{U}$,

$$
\begin{gathered}
H_{D}(\mathbf{1}, \mathbf{2},-1 / 2) \equiv\left(H_{d}=\left(\begin{array}{c}
H_{d}^{0} \\
H_{d}^{-}
\end{array}\right), \widetilde{H}_{d}=\left(\begin{array}{c}
\widetilde{H}_{d}^{0} \\
\widetilde{H}_{d}^{-}
\end{array}\right)\right), \\
H_{U}(\mathbf{1}, \mathbf{2}, 1 / 2) \equiv\left(H_{u}=\left(\begin{array}{c}
H_{u}^{+} \\
H_{u}^{0}
\end{array}\right), \widetilde{H}_{u}=\left(\begin{array}{c}
\widetilde{H}_{u}^{+} \\
\widetilde{H}_{u}^{0}
\end{array}\right)\right),
\end{gathered}
$$

which allows for the cancellation of chiral anomalies and the generation of masses for all up-type and down-type particles.

All kinetic and gauge-interaction Lagrangian terms are fixed by gauge and SUSY invariance, and can be casted very compactly within the superspace formalism [6-8],

$$
\begin{aligned}
\mathcal{L}_{\text {kin }}= & \sum_{\Phi}\left[\Phi^{\dagger}\left(e^{-2 g_{y} V_{B}} e^{-2 g_{w} V_{W}} e^{-2 g_{s} V_{G}}\right) \Phi\right]_{\theta \cdot \theta \bar{\theta} \cdot \bar{\theta}} \\
& +\left\{\left[\frac{1}{4} W_{B}^{\alpha} W_{B \alpha}+\frac{1}{16 g_{w}^{2}} W_{W k}^{\alpha} W_{W \alpha}^{k}+\frac{1}{16 g_{s}^{2}} W_{G a}^{\alpha} W_{G \alpha}^{a}\right]_{\theta \cdot \theta}+\text { h.c. }\right\},
\end{aligned}
$$


where the notation $[.]_{X}$ indicates that after expanding the superfield inside the bracket in terms of the Grassmanian variables $\theta$ and $\bar{\theta}$, only the $X$-component is retained. The first line of the Lagrangian refers to the chiral sector of the theory and includes a sum upon the superfields associated with all the previously-introduced chiral supermultiplets. The vector superfields appearing in the exponents are considered contracted with the relevant representation matrices, and $g_{y}, g_{w}$ and $g_{s}$ denote the hypercharge, weak and strong coupling constants. The last line of this Lagrangian describes the gauge sector, and involves squares of the superfield strength tensors associated with the three gauge subgroups, the summed spin indices $\alpha$ being indicated explicitly.

Assuming $R$-parity conservation to avoid the presence of baryon- and lepton-number violating interactions, which will challenge the experimental observations [60], the superpotential interactions include Yukawa couplings, generating in particular the SM quark and lepton masses, as well as the off-diagonal Higgs mass-mixing $\mu$ term. The corresponding Lagrangian in superspace is written as

$$
\mathcal{L}_{\text {superW }}=\left[W_{\operatorname{MSSM}}(\Phi)\right]_{\theta \cdot \theta}+\text { h.c. }
$$

where the superpotential $W_{\operatorname{MSSM}}(\Phi)$ is given, in the flavour space and with all flavour indices understood for clarity, by

$$
W_{\operatorname{MSSM}}(\Phi)=U_{R} \mathbf{y}^{\mathbf{u}} Q_{L} H_{U}-D_{R} \mathbf{y}^{\mathbf{d}} Q_{L} H_{D}+E_{R} \mathbf{y}^{\mathbf{e}} L_{L} H_{D}+\mu H_{U} H_{D}
$$

In this expression, the matrices $\mathbf{y}^{\mathbf{u}}, \mathbf{y}^{\mathbf{d}}$ and $\mathbf{y}^{\mathbf{e}}$ are the usual $3 \times 3$ Yukawa matrices.

The expansion of the above Lagrangian in terms of the component fields relies on standard techniques detailed, e.g., in refs. [61, 62], and allows for the extraction of the SUSY-conserving part of the MSSM Lagrangian. The results read

$$
\begin{aligned}
& \mathcal{L}_{\mathrm{MSSM}}^{(\mathrm{SUSY})}=\sum_{k}\left[-\frac{1}{4} V_{k}^{\mu \nu} V_{\mu \nu}^{k}+\frac{i}{2}\left(\widetilde{V}^{k} \sigma^{\mu} D_{\mu} \overline{\widetilde{V}}_{k}-D_{\mu} \widetilde{V}^{k} \sigma^{\mu} \overline{\widetilde{V}}_{k}\right)\right] \\
& +\sum_{i}\left[D_{\mu} \phi_{i}^{\dagger} D^{\mu} \phi^{i}+\frac{i}{2}\left(\psi^{i} \sigma^{\mu} D_{\mu} \bar{\psi}_{i}-D_{\mu} \psi^{i} \sigma^{\mu} \bar{\psi}_{i}\right)+i \sqrt{2} \sum_{k}\left(g_{k} \overline{\widetilde{V}}^{k} \cdot \bar{\psi}_{i} T_{k} \phi^{i}+\text { h.c. }\right)\right] \\
& -\frac{1}{2} \sum_{i, j}\left[\frac{\partial^{2} W_{\mathrm{MSSM}}(\phi)}{\partial \phi^{i} \partial \phi^{j}} \psi^{i} \cdot \psi^{j}+\text { h.c. }\right] \\
& -\sum_{i} \frac{\partial W_{\mathrm{MSSM}}(\phi)}{\partial \phi^{i}} \frac{\partial W_{\mathrm{MSSM}}^{\star}\left(\phi^{\dagger}\right)}{\partial \phi_{i}^{\dagger}}-\frac{1}{2} \sum_{k}\left[\left(g_{k} \sum_{j}\left[\phi_{j}^{\dagger} T^{k} \phi^{j}\right]\right)\left(g_{k} \sum_{i}\left[\phi_{i}^{\dagger} T_{k} \phi^{i}\right]\right)\right]
\end{aligned}
$$

where all fermionic fields are two-component left-handed $(\psi$ and $\widetilde{V})$ or right-handed $(\bar{\psi}$ and $\overline{\widetilde{V}})$ spinors, the dot products are invariant products in spin space, $\sigma_{\mu}=\left(1, \sigma^{i}\right)$ consists of one of the possible four-vectors built upon the Pauli matrices and we used the usual gauge field strength tensor $V_{\mu \nu}$ and covariant derivatives $D_{\mu}$ taken in the appropriate representation of the gauge group. The summation over $k$ refers to the three MSSM gauge groups (the generic notation $T_{k}$ and $g_{k}$ being used for the gauge-group representation matrices and coupling constants), whereas we use the indices $i$ and $j$ and the generic $(\phi, \psi)$ 
notation for the sum over the scalar and fermionic component of the model supermutiplets. In addition, the gaugino-scalar-fermion interactions are included in the last term of the second line, the third line is constituted of the Yukawa interactions deduced from the superpotential, and the scalar potential is shown in the last line of the above Lagrangian.

\subsubsection{Soft supersymmetry breaking and particle mixings}

As for any phenomenologically realistic SUSY theory, the MSSM exhibits soft SUSY breaking to introduce a mass splitting between a SM particle and its superpartner. We supplement the Lagrangian of eq. (2.7) by all possible soft terms breaking SUSY explicitly [63],

$$
\begin{aligned}
\mathcal{L}_{\mathrm{MSSM}}^{\text {(soft) }}= & \frac{1}{2}\left[M_{1} \widetilde{B} \cdot \widetilde{B}+M_{2} \widetilde{W} \cdot \widetilde{W}+M_{3} \widetilde{G} \cdot \widetilde{G}+\text { h.c. }\right]-\tilde{q}_{L}^{\dagger} \mathbf{m}_{\tilde{\mathbf{Q}}}^{\mathbf{2}} \tilde{q}_{L}-\tilde{u}_{R} \mathbf{m}_{\tilde{\mathbf{U}}}^{\mathbf{2}} \tilde{u}_{R}^{\dagger} \\
& -\tilde{d}_{R} \mathbf{m}_{\tilde{\mathbf{D}}}^{\mathbf{2}} \tilde{d}_{R}^{\dagger}-\tilde{\ell}_{L}^{\dagger} \mathbf{m}_{\tilde{\mathbf{L}}}^{2} \tilde{\ell}_{L}-\tilde{e}_{R} \mathbf{m}_{\tilde{\mathbf{E}}}^{\mathbf{2}} \tilde{e}_{R}^{\dagger}-m_{H_{u}}^{2} H_{u}^{\dagger} H_{u}-m_{H_{d}}^{2} H_{d}^{\dagger} H_{d} \\
& -\left[\tilde{u}_{R}^{\dagger} \mathbf{T}^{\mathbf{u}} \tilde{q}_{L} \cdot H_{u}-\tilde{d}_{R}^{\dagger} \mathbf{T}^{\mathbf{d}} \tilde{q}_{L} \cdot H_{d}-\tilde{e}_{R}^{\dagger} \mathbf{T}^{\mathbf{e}} \tilde{\ell}_{L} \cdot H_{d}+b H_{u} \cdot H_{d}+\text { h.c. }\right] .
\end{aligned}
$$

The soft SUSY-breaking Lagrangian firstly includes mass terms for the gauginos, the mass parameters of the $\mathrm{U}(1)_{Y}, \mathrm{SU}(2)_{L}$ and $\mathrm{SU}(3)_{c}$ gauginos being denoted by $M_{1}, M_{2}$ and $M_{3}$. The following seven terms consist of mass terms for all scalar fields, the parameters $\mathbf{m}_{\tilde{\mathbf{Q}}}$, $\mathbf{m}_{\tilde{\mathbf{L}}}, \mathbf{m}_{\tilde{\mathbf{U}}}, \mathbf{m}_{\tilde{\mathbf{D}}}$ and $\mathbf{m}_{\tilde{\mathbf{E}}}$ being $3 \times 3$ Hermitian matrices in the flavour space and $m_{H_{u}}$ and $m_{H_{d}}$ are the two Higgs mass parameters. In addition, bilinear and trilinear soft multiscalar interactions can be deduced from the form of the superpotential, the corresponding coupling strengths being organised in the three $3 \times 3 \mathbf{T} \mathbf{u}, \mathbf{T}^{\mathbf{d}}$ and $\mathbf{T}^{\mathbf{e}}$ matrices (in the flavour space) and the complex number $b$ being related to the soft SUSY-breaking Higgs mixing term.

At the minimum of the scalar potential, the neutral components of both Higgs doublets $H_{u}^{0}$ and $H_{d}^{0}$ get non-vanishing vacuum expectation values and the electroweak symmetry is broken. As a result, the electroweak vector bosons $B_{\mu}$ and $W_{\mu}^{3}$ mix into the massless photon $A_{\mu}$ and massive $Z_{\mu}$ states,

$$
\left(\begin{array}{c}
A_{\mu} \\
Z_{\mu}
\end{array}\right)=\left(\begin{array}{cc}
\cos \theta_{w} & \sin \theta_{w} \\
-\sin \theta_{w} & \cos \theta_{w}
\end{array}\right)\left(\begin{array}{c}
B_{\mu} \\
W_{\mu}^{3}
\end{array}\right),
$$

where we have introduced the electroweak mixing angle $\theta_{w}$. As in the SM, the charged weak boson physical states are defined by diagonalising the third generator of $\mathrm{SU}(2)$ in the adjoint representation, which gives rise to

$$
W_{\mu}^{ \pm}=\frac{1}{\sqrt{2}}\left(W_{\mu}^{1} \mp i W_{\mu}^{2}\right) .
$$

The eight degrees of freedom included within the two Higgs doublets give rise to three Goldstone bosons $G^{ \pm}$and $G^{0}$ that become the longitudinal modes of the weak gauge bosons and five physical Higgs bosons $h^{0}, H^{0}, A^{0}$ and $H^{ \pm}$defined by diagonalising the Higgs sector,

$$
\begin{aligned}
& H_{u}^{0}=\frac{v_{u}}{\sqrt{2}}+\cos \alpha h^{0}+\sin \alpha H^{0}+i \cos \beta A^{0}+i \sin \beta G^{0}, \quad H_{u}^{+}=\cos \beta H^{+}+\sin \beta G^{+}, \\
& H_{d}^{0}=\frac{v_{d}}{\sqrt{2}}-\sin \alpha h^{0}+\cos \alpha H^{0}+i \sin \beta A^{0}-i \cos \beta G^{0}, \quad H_{d}^{-}=\sin \beta H^{-}-\cos \beta G^{-},
\end{aligned}
$$


where we have introduced the Higgs mixing angles $\alpha$ and $\beta$, the tangent of the latter being given by the ratio of the vacuum expectation values of the neutral Higgs gauge eigenstates, $\tan \beta=v_{u} / v_{d}$.

Mixings in the fermionic electroweak sector are also induced by the breaking of the electroweak symmetry. In the neutral sector, the gaugino and Higgsino gauge eigenstates mix into the physical neutralino eigenstates $\chi_{i}^{0}$ (with $i=1,2,3,4$ ), whereas in the charged sector, they mix into the two chargino states $\chi_{i}^{ \pm}$(with $i=1,2$ ),

$$
\left(\begin{array}{c}
\chi_{1}^{0} \\
\chi_{2}^{0} \\
\chi_{3}^{0} \\
\chi_{4}^{0}
\end{array}\right)=N\left(\begin{array}{c}
i \tilde{B} \\
i \tilde{W}^{3} \\
\tilde{H}_{d}^{0} \\
\tilde{H}_{u}^{0}
\end{array}\right), \quad\left(\begin{array}{c}
\chi_{1}^{+} \\
\chi_{2}^{+}
\end{array}\right)=V\left(\begin{array}{c}
i \tilde{W}^{+} \\
\tilde{H}_{u}^{+}
\end{array}\right) \quad \text { and } \quad\left(\begin{array}{c}
\chi_{1}^{-} \\
\chi_{2}^{-}
\end{array}\right)=U\left(\begin{array}{c}
i \tilde{W}^{-} \\
\tilde{H}_{d}^{-}
\end{array}\right) .
$$

In those relations, the mixing matrices $N, U$ and $V$ are unitary and allow one to diagonalise the neutral and charged electroweakino mass matrices.

As in the SM, the diagonalisation of the quark sector requires four unitary matrices $V_{u}, V_{d}, U_{u}$ and $U_{d}$ and the one of the lepton sector relies on three unitary rotation matrices $V_{e}, V_{\nu}$ and $U_{e}$ as we have omitted the right-handed neutrino (super)fields in the model definition. This leads to the following field redefinitions in the flavour space,

$$
\begin{array}{rlrlrl}
d_{L} \rightarrow V_{d} d_{L}, & d_{R}^{c} \rightarrow d_{R}^{c} U_{d}^{\dagger}, & u_{L} \rightarrow V_{u} u_{L} & \text { and } & u_{R}^{c} \rightarrow u_{R}^{c} U_{u}^{\dagger}, \\
e_{L} \rightarrow V_{e} e_{L}, & e_{R}^{c} \rightarrow e_{R}^{c} U_{e}^{\dagger} & \text { and } & \nu_{L} \rightarrow V_{\nu} \nu_{L},
\end{array}
$$

and we follow the traditional approach of casting these rotations through a redefinition of the left-handed down-type quark field only via the CKM matrix $V_{\mathrm{CKM}}$,

$$
d_{L} \rightarrow V_{\mathrm{CKM}} d_{L}=V_{u}^{\dagger} V_{d} d_{L}
$$

In agreement with SUSY, these field redefinitions are promoted to the superfield level so that one must consider an extra rotation acting on the basis of left-handed down-type squarks similar to the one of eq. (2.14),

$$
\tilde{d}_{L} \rightarrow V_{\mathrm{CKM}} \tilde{d}_{L}
$$

The two field redefinitions of eq. (2.14) and eq. (2.15) define the so-called super-CKM basis [64] in which the resulting $6 \times 6$ squark mass matrices are non-diagonal. Following the SUSY Les Houches Accord (SLHA) conventions [65], the superpotential and soft parameters are redefined according to

$$
\begin{aligned}
\mathbf{y}^{\mathbf{u}} \rightarrow \hat{\mathbf{y}}^{\mathbf{u}}=U_{u}^{\dagger} \mathbf{y}^{\mathbf{u}} V_{u}, & \mathbf{y}^{\mathbf{d}} \rightarrow \hat{\mathbf{y}}^{\mathrm{d}} V_{d}^{\dagger} V_{u}=U_{d}^{\dagger} \mathbf{y}^{\mathbf{d}} V_{u}, & \mathbf{y}^{\mathbf{e}} \rightarrow \hat{\mathbf{y}}^{\mathbf{e}}=U_{e}^{\dagger} \mathbf{y}^{\mathbf{e}} V_{e}, \\
\mathbf{T}^{\mathbf{u}} \rightarrow \hat{\mathbf{T}}^{\mathbf{u}}=U_{u}^{\dagger} \mathbf{T}^{\mathbf{u}} V_{u}, & \mathbf{T}^{\mathbf{d}} \rightarrow \hat{\mathbf{T}}^{\mathrm{d}} \mathbf{V}_{\mathrm{CKM}}^{\dagger}=U_{d}^{\dagger} \mathbf{T}^{\mathbf{d}} V_{u}, & \mathbf{T}^{\mathbf{e}} \rightarrow \hat{\mathbf{T}}^{\mathbf{e}}=U_{e}^{\dagger} \mathbf{T}^{\mathbf{e}} V_{e}, \\
\mathbf{m}_{\tilde{\mathbf{Q}}}^{2} \rightarrow V_{\mathrm{CKM}} \hat{\mathbf{m}}_{\tilde{\mathbf{Q}}}^{2} V_{\mathrm{CKM}}^{\dagger}, & \mathbf{m}_{\tilde{\mathbf{U}}}^{2} \rightarrow \hat{\mathbf{m}}_{\tilde{\mathbf{U}}}^{2}=U_{u}^{\dagger} \mathbf{m}_{\tilde{\mathbf{U}}}^{2} U_{u}, & \mathbf{m}_{\tilde{\mathbf{D}}}^{2} \rightarrow \hat{\mathbf{m}}_{\tilde{\mathbf{D}}}^{2}=U_{d}^{\dagger} \mathbf{m}_{\tilde{\mathbf{D}}}^{2} U_{d}, \\
\mathbf{m}_{\tilde{\mathbf{L}}}^{2} \rightarrow \hat{\mathbf{m}}_{\tilde{\mathbf{L}}}^{2}=V_{e}^{\dagger} \mathbf{m}_{\tilde{\mathbf{L}}}^{2} V_{e}, & \mathbf{m}_{\tilde{\mathbf{E}}}^{2} \rightarrow \hat{\mathbf{m}}_{\tilde{\mathbf{E}}}^{2}=U_{e}^{\dagger} \mathbf{m}_{\tilde{\mathbf{E}}}^{2} U_{e}, &
\end{aligned}
$$

where the hatted quantities refer to the new free parameters of the theory. The matrices $\hat{\mathbf{y}}^{\mathbf{u}}, \hat{\mathbf{y}}^{\mathbf{d}}$ and $\hat{\mathbf{y}}^{\mathbf{e}}$ are diagonal and real $3 \times 3$ matrices in the flavour space, whereas all the other 
matrices are in principle possibly flavour-violating and $C P$-violating. We however assume a constrained realisation of the MSSM in which organising principles of the soft terms forbid any source of flavour and $C P$ violation on top of those inherent to the CKM matrix. In this case, all subsequent flavour-violating effects in the squark sector are small, and each squark flavour turns out to be approximately aligned with the associated quark flavour.

Following the SLHA conventions and the standard MSSM literature, the $\hat{\mathbf{T}}^{\mathrm{f}}$ matrices are decomposed as

$$
\hat{\mathbf{T}}^{\mathrm{f}}=\mathbf{A}^{\mathrm{f}} \hat{\mathbf{y}}^{\mathrm{f}}
$$

where the overall strengths of the trilinear scalar interactions for a (s)fermion species $f$ are embedded into the three $3 \times 3 \mathbf{A}^{\mathbf{f}}$ (diagonal and real) matrices in the flavour space. As a consequence and for not too extreme values of the coupling strengths $\mathbf{A}^{\mathbf{f}}$, typical MSSM scenarios only exhibit a flavour-conserving mixing of the third generation sfermions, any other mixing being subdominant and negligible. Such a mixing is modelled through the stop $\left(\theta_{\tilde{t}}\right)$, sbottom $\left(\theta_{\tilde{b}}\right)$ and stau $\left(\theta_{\tilde{\tau}}\right)$ mixing angles, and the stop $\left(\tilde{t}_{1}, \tilde{t}_{2}\right)$, sbottom $\left(\tilde{b}_{1}\right.$, $\left.\tilde{b}_{2}\right)$ and stau $\left(\tilde{\tau}_{1}, \tilde{\tau}_{2}\right)$ mass-ordered physical states are related to the corresponding gauge eigenstates as

$$
\begin{aligned}
& \left(\begin{array}{c}
\tilde{t}_{1} \\
\tilde{t}_{2}
\end{array}\right)=S_{\tilde{t}}\left(\begin{array}{c}
\tilde{t}_{L} \\
\tilde{t}_{R}
\end{array}\right)=\left(\begin{array}{c}
\cos \theta_{\tilde{t}} \sin \theta_{\tilde{t}} \\
-\sin \theta_{\tilde{t}} \cos \theta_{\tilde{t}}
\end{array}\right)\left(\begin{array}{c}
\tilde{t}_{L} \\
\tilde{t}_{R}
\end{array}\right), \quad\left(\begin{array}{c}
\tilde{b}_{1} \\
\tilde{b}_{2}
\end{array}\right)=S_{\tilde{b}}\left(\begin{array}{c}
\tilde{b}_{L} \\
\tilde{b}_{R}
\end{array}\right)=\left(\begin{array}{cc}
\cos \theta_{\tilde{b}} \sin \theta_{\tilde{b}} \\
-\sin \theta_{\tilde{b}} \cos \theta_{\tilde{b}}
\end{array}\right)\left(\begin{array}{c}
\tilde{b}_{L} \\
\tilde{b}_{R}
\end{array}\right), \\
& \left(\begin{array}{c}
\tilde{\tau}_{1} \\
\tilde{\tau}_{2}
\end{array}\right)=S_{\tilde{\tau}}\left(\begin{array}{c}
\tilde{\tau}_{L} \\
\tilde{\tau}_{R}
\end{array}\right)=\left(\begin{array}{c}
\cos \theta_{\tilde{\tau}} \sin \theta_{\tilde{\tau}} \\
-\sin \theta_{\tilde{\tau}} \cos \theta_{\tilde{\tau}}
\end{array}\right)\left(\begin{array}{c}
\tilde{\tau}_{L} \\
\tilde{\tau}_{R}
\end{array}\right) .
\end{aligned}
$$

\subsubsection{Two-component and four-component fermions}

All the two-component fermionic fields introduced so far are finally combined to form Dirac and Majorana spinors $\Psi$, that are the fermion representations supported at the level of the Monte Carlo event generators which we plan to use for our phenomenological study. The SM, gluino, chargino and neutralino four-component fermions are defined by

$$
\begin{aligned}
\Psi_{u}=\left(\begin{array}{c}
u_{L} \\
\bar{u}_{R}^{c}
\end{array}\right), & \Psi_{d}=\left(\begin{array}{c}
d_{L} \\
\bar{d}_{R}^{c}
\end{array}\right), & \Psi_{e}=\left(\begin{array}{c}
e_{L} \\
\bar{e}_{R}^{c}
\end{array}\right), \quad \Psi_{\nu}=\left(\begin{array}{c}
\nu_{L} \\
0
\end{array}\right), \\
\Psi_{\chi^{0}}=\left(\begin{array}{c}
\chi^{0} \\
\bar{\chi}^{0}
\end{array}\right), & \Psi_{\chi^{ \pm}}=\left(\begin{array}{c}
\chi^{ \pm} \\
\bar{\chi}^{\mp}
\end{array}\right), & \Psi_{\tilde{g}}=\left(\begin{array}{c}
i \tilde{g} \\
-i \overline{\tilde{g}}
\end{array}\right) .
\end{aligned}
$$

Although the tree-level form of all mixing matrices introduced so far can be easily calculated from the Lagrangians of eq. (2.7) and eq. (2.8), loop correction effects are important. Oneloop and known two-loop contributions are hence in general included in all available MSSM spectrum generators [66-69].

\subsection{Renormalisation}

\subsubsection{Generalities}

Focusing in the following on NLO calculations in $\alpha_{S}$, we rotate the Lagrangians of eqs. (2.7) and (2.8) to the mass basis and omit from the discussion any term that is irrelevant with 
respect to the strong interaction,

$$
\begin{aligned}
\mathcal{L}_{\mathrm{MSSM}}^{(\mathrm{QCD})}= & \mathcal{L}_{\mathrm{SM}}^{(\mathrm{QCD})}+\sum_{\tilde{q}_{k}}\left[D_{\mu} \tilde{q}_{k}^{\dagger} D^{\mu} \tilde{q}_{k}-m_{\tilde{q}_{k}}^{2} \tilde{q}_{k}^{\dagger} \tilde{q}_{k}\right]+\frac{i}{2} \bar{\Psi}_{\tilde{g}} \not D \Psi_{\tilde{g}}-\frac{1}{2} m_{\tilde{g}} \bar{\Psi}_{\tilde{g}} \Psi_{\tilde{g}} \\
& -\frac{g_{s}^{2}}{2}\left\{\sum_{q}\left[\left(S_{\tilde{q}}\right)_{j 2}\left(S_{\tilde{q}}\right)_{2 i}^{*} \tilde{q}_{j}^{\dagger} T \tilde{q}_{i}-\left(S_{\tilde{q}}\right)_{j 1}\left(S_{\tilde{q}}\right)_{1 i}^{*} \tilde{q}_{j}^{\dagger} T \tilde{q}_{i}\right]\right\}^{2} \\
& +\sqrt{2} g_{s} \sum_{q}\left[-\left(S_{\tilde{q}}\right)_{j 1} \tilde{q}_{j}^{\dagger} T\left(\bar{\Psi}_{\tilde{g}} P_{L} \Psi_{q}\right)+\left(\bar{\Psi}_{q} P_{L} \Psi_{\tilde{g}}\right) T\left(S_{\tilde{q}}\right)_{2 j}^{*} \tilde{q}_{j}+\text { h.c. }\right]+\ldots
\end{aligned}
$$

In this expression, $\mathcal{L}_{\mathrm{SM}}^{(\mathrm{QCD})}$ denotes the $\mathrm{QCD}$ part of the SM Lagrangian involving quarks and gluons, the sum over $\tilde{q}_{k}$ refers to a sum over all twelve squark mass-eigenstates $\left(\tilde{u}_{L}\right.$, $\left.\tilde{c}_{L}, \tilde{t}_{1}, \tilde{u}_{R}, \tilde{c}_{R}, \tilde{t}_{2}, \tilde{d}_{L}, \tilde{s}_{L}, \tilde{b}_{1}, \tilde{d}_{R}, \tilde{s}_{R}, \tilde{b}_{2}\right)$ of masses $m_{\tilde{q}_{k}}$ and the sums over $q$ refer to sums over all six quark flavours. In the former sums, the mixing matrices associated with the first and second generation squarks are taken as $2 \times 2$ identity matrices, so that the first and second generation mass-eigenstates $\tilde{q}_{1}$ and $\tilde{q}_{2}$ are subsequently identified with the left-handed and right-handed squarks $\tilde{q}_{L}$ and $\tilde{q}_{R}$, respectively. In addition, $m_{\tilde{g}}$ stands for the gluino mass, $T$ for the fundamental representation matrices of $\mathrm{SU}(3), P_{L, R}$ for the lefthanded and right-handed chirality projectors, $g_{s}=\sqrt{4 \pi \alpha_{S}}$ for the strong coupling constant and the covariant derivatives are restricted to their QCD component.

Ultraviolet divergences appearing at the one-loop level are absorbed into the counterterms generated by the renormalisation of the above Lagrangian. Following the usual procedure, all bare bosonic fields $\Phi$ and fermionic fields $\Psi$ are replaced by their renormalised counterparts,

$$
\Phi \rightarrow\left[1+\frac{1}{2} \delta Z_{\Phi}\right] \Phi \quad \text { and } \quad \Psi \rightarrow\left[1+\frac{1}{2} \delta Z_{\Psi}^{L} P_{L}+\frac{1}{2} \delta Z_{\Psi}^{R} P_{R}\right] \Psi
$$

with the exception of third generation squarks for which matrix renormalisation is in order as they mix,

$$
\left(\begin{array}{c}
\tilde{q}_{1} \\
\tilde{q}_{2}
\end{array}\right) \rightarrow\left(\begin{array}{c}
\tilde{q}_{1} \\
\tilde{q}_{2}
\end{array}\right)+\frac{1}{2}\left(\begin{array}{ll}
\delta Z_{\tilde{q}, 11} & \delta Z_{\tilde{q}, 12} \\
\delta Z_{\tilde{q}, 21} & \delta Z_{\tilde{q}, 22}
\end{array}\right)\left(\begin{array}{c}
\tilde{q}_{1} \\
\tilde{q}_{2}
\end{array}\right) \quad \text { with } \quad \tilde{q}=\tilde{b}, \tilde{t} .
$$

Although the structure of the gluino-squark-quark interactions (the last line of eq. (2.20)) could induce the mixing of any squark flavour at the one-loop level, those effects are proportional to the corresponding quark masses. Considering $n_{l f}=4$ flavours of massless quarks, the first two generations are kept non-mixing so that gauge and mass eigenstates are equivalent. In addition, the bare parameters of the MSSM Lagrangian, generically denoted by $y$ (for couplings) and $m$ (for masses), are renormalised as

$$
y \rightarrow y+\delta y \quad \text { and } \quad m \rightarrow m+\delta m .
$$

In this work, we calculate the various renormalisation constants appearing in the renormalisation procedure of the Lagrangian of eq. (2.20) in the on-shell (OS) scheme where the input parameters are physical observables such as the physical particle masses. There is 
however no unique definition of such a scheme in SUSY by virtue of existing interrelations between various mass and coupling parameters, which will be addressed in section 2.2.4. Fermion self-energy corrections $\Sigma(p)$ are decomposed in terms of independent Lorentz structures,

$$
\Sigma(p)=-i\left[\Sigma_{L}^{V}\left(p^{2}\right) \not p P_{L}+\Sigma_{R}^{V}\left(p^{2}\right) \not p P_{R}+\Sigma_{L}^{S}\left(p^{2}\right) P_{L}+\Sigma_{R}^{S}\left(p^{2}\right) P_{R}\right],
$$

from which the OS fermionic wave-function renormalisation constants $\delta Z_{f}^{L, R}$ and mass renormalisation constant $\delta m_{f}$ can be deduced. Imposing that the renormalised mass is the pole of the propagator and that the residue of the propagator pole equals 1 , we get

$$
\begin{aligned}
\delta Z_{f}^{L, R} & =\Sigma_{L, R}^{V}\left(m_{f}^{2}\right)+m_{f}^{2}\left[\Sigma_{L}^{\prime}\left(m_{f}^{2}\right)+\Sigma_{R}^{\prime V}\left(m_{f}^{2}\right)\right]+m_{f}\left[\Sigma_{L}^{\prime S}\left(m_{f}^{2}\right)+\Sigma_{R}^{\prime S}\left(m_{f}^{2}\right)\right] \\
\delta m_{f} & =-\frac{1}{2}\left[\Sigma_{L}^{V}\left(m_{f}^{2}\right)+\Sigma_{R}^{V}\left(m_{f}^{2}\right)\right]-\frac{1}{2}\left[\Sigma_{L}^{S}\left(m_{f}^{2}\right)+\Sigma_{R}^{S}\left(m_{f}^{2}\right)\right]
\end{aligned}
$$

where the prime denotes a derivative with respect to $p^{2}$. Gauge-boson self-energy corrections $D_{\mu \nu}(p)$ are reduced, in the case of the gluon (that is the only relevant gauge boson as long as only $\alpha_{S}$ corrections are concerned), to their purely transverse component,

$$
D_{\mu \nu}(p)=i\left[\eta^{\mu \nu}-\frac{p_{\mu} p_{\nu}}{p^{2}}\right] D^{T}\left(p^{2}\right) .
$$

The wave-function renormalisation constant $\delta Z_{g}$ is obtained after imposing OS renormalisation conditions, and reads

$$
\delta Z_{g}=D^{\prime T}(0)
$$

Finally, as above-mentioned, the scalar quark sector of the theory relies on matrix renormalisation, any off-diagonal element being vanishing in the case of the first and second generation non-mixing squarks. We rewrite the scalar self-energy correction $\Pi_{i j}(p)$ in the following form,

$$
\Pi_{i j}(p)=-i \Pi_{i j}^{S}\left(p^{2}\right),
$$

where the indices $i$ and $j$ are either 1 or 2 and are related to the nature of the external squark eigenstates. This allows for the derivation of the diagonal $\left(\delta Z_{i i}\right)$ and non-diagonal $\left(\delta Z_{i j}\right)$ squark wave-function renormalisation constants,

$$
\delta Z_{i i}=\Pi_{i i}^{\prime S}\left(m_{i}^{2}\right) \quad \text { and } \quad \delta Z_{i j}=\delta Z_{j i}=\frac{\Pi_{i j}^{S}\left(m_{j}^{2}\right)-\Pi_{i j}^{S}\left(m_{i}^{2}\right)}{m_{j}^{2}-m_{i}^{2}},
$$

where $m_{i}^{2}$ indicates the squared mass of the $i^{\text {th }}$ eigenstate, as well as of the mass renormalisation constant,

$$
\delta m_{i}^{2}=-\Pi_{i i}^{S}\left(m_{i}^{2}\right) .
$$

We do not address the complex-mass renormalisation scheme in this paper, so that we kept implicit that only the real-part of the self-energies is considered in the above expressions. The case of complex-mass renormalisation conditions is especially delicate in the case of NLO computations within SUSY theories because their mass spectrum is arbitrary to a large extent thus making it necessary to implement the most general analytic continuation of the two-point functions [70]. 


\subsubsection{The Standard Model sector}

Beginning with the SM sector, the wave-function renormalisation constants $\delta Z_{q}^{L, R}$ of the massless quarks $q=u, d, s, c$ and $\delta Z_{Q}^{L, R}$ of the massive bottom and top quarks $Q=b, t$, as well as the one of the gluon $\delta Z_{g}$ are given by

$$
\begin{aligned}
\delta Z_{g}= & -\frac{g_{s}^{2}}{24 \pi^{2}}\left[\sum_{Q=b, t}\left\{B_{0}\left(0, m_{Q}^{2}, m_{Q}^{2}\right)+2 m_{Q}^{2} B_{0}^{\prime}\left(0, m_{Q}^{2}, m_{Q}^{2}\right)-\frac{1}{3}\right\}+n_{c}\left\{B_{0}\left(0, m_{\tilde{g}}^{2}, m_{\tilde{g}}^{2}\right)\right.\right. \\
& \left.\left.+2 m_{\tilde{g}}^{2} B_{0}^{\prime}\left(0, m_{\tilde{g}}^{2}, m_{\tilde{g}}^{2}\right)-\frac{1}{3}\right\}+\sum_{\tilde{q}_{k}}\left\{\frac{1}{4} B_{0}\left(0, m_{\tilde{q}_{k}}^{2}, m_{\tilde{q}_{k}}^{2}\right)-m_{\tilde{q}}^{2} B_{0}^{\prime}\left(0, m_{\tilde{q}_{k}}^{2}, m_{\tilde{q}_{k}}^{2}\right)+\frac{1}{6}\right\}\right], \\
\delta Z_{q}^{L, R}= & \frac{g_{s}^{2} C_{F}}{8 \pi^{2}} B_{1}\left(0 ; m_{\tilde{g}}^{2}, m_{\tilde{q}_{L, R}}^{2}\right), \\
\delta Z_{Q}^{L, R}= & \frac{g_{s}^{2} C_{F}}{16 \pi^{2}}\left[1+2 B_{1}\left(m_{Q}^{2} ; m_{Q}^{2}, 0\right)+4 m_{Q}^{2} B_{1}^{\prime}\left(m_{Q}^{2} ; m_{Q}^{2}, 0\right)+8 m_{Q}^{2} B_{0}^{\prime}\left(m_{Q}^{2} ; m_{Q}^{2}, 0\right)\right. \\
& +2 \sum_{i=1}^{2} B_{1}\left(m_{Q}^{2} ; m_{\tilde{g}}^{2}, m_{\tilde{Q}_{i}}^{2}\right)\left|\left(S_{\tilde{Q}}\right)_{j 1,2}\right|^{2}+2 m_{Q}^{2} \sum_{i=1}^{2} B_{1}^{\prime}\left(m_{Q}^{2} ; m_{\tilde{g}}^{2}, m_{\tilde{Q}_{i}}^{2}\right) \\
& \left.+4 m_{Q}^{2} \sum_{i=1}^{2} B_{0}^{\prime}\left(m_{Q}^{2} ; m_{\tilde{g}}^{2}, m_{\tilde{Q}_{i}}^{2}\right)\left(S_{\tilde{Q}}\right)_{j 1}^{*}\left(S_{\tilde{Q}}\right)_{j 2}\right]
\end{aligned}
$$

where the $B_{0}$ and $B_{1}$ functions and their derivatives are the real part of the usual twopoint Passarino-Veltman integrals [71] collected in appendix A. Moreover, $n_{c}=3$ and $C_{F}=\left(n_{c}^{2}-1\right) /\left(2 n_{c}\right)$ stand respectively for the number of colours and for the quadratic Casimir invariant connected with the fundamental representation of $\mathrm{SU}(3)$, and the sum upon $\tilde{q}_{k}$ refers to a sum over all squark states. In addition, the bottom and top mass OS renormalisation constants $\delta m_{Q}$ (with $Q=b, t$ ) are given by

$$
\begin{aligned}
\delta m_{Q}=-\frac{g_{s}^{2} C_{F} m_{Q}}{16 \pi^{2}}[ & -1+4 B_{0}\left(m_{Q}^{2} ; m_{Q}^{2}, 0\right)+2 B_{1}\left(m_{Q}^{2} ; m_{Q}^{2}, 0\right)+\sum_{i=1}^{2} B_{1}\left(m_{Q}^{2} ; m_{\tilde{g}}^{2}, m_{\tilde{Q}_{i}}^{2}\right) \\
& \left.+\sum_{i=1}^{2}\left(S_{\tilde{Q}}\right)_{j 1}^{*}\left(S_{\tilde{Q}}\right)_{j 2} B_{0}\left(m_{Q}^{2} ; m_{\tilde{g}}^{2}, m_{\tilde{Q}_{i}}^{2}\right)\right]
\end{aligned}
$$

\subsubsection{Gluino renormalisation}

Gluino renormalisation in the OS scheme is standard, and the corresponding wave-function renormalisation constant $\delta Z_{\tilde{g}} \equiv \delta Z_{\tilde{g}}^{L}=\delta Z_{\tilde{g}}^{R}$ (as the gluino is a Majorana fermion) and mass renormalisation constant $\delta m_{\tilde{g}}$ read

$$
\begin{aligned}
\delta Z_{\tilde{g}}=\frac{g_{s}^{2}}{16 \pi^{2}} & {\left[n_{c}+2 n_{c} B_{1}\left(m_{\tilde{g}}^{2} ; m_{\tilde{g}}^{2}, 0\right)+8 n_{c} m_{\tilde{g}}^{2} B_{0}^{\prime}\left(m_{\tilde{g}}^{2} ; m_{\tilde{g}}^{2}, 0\right)+4 n_{c} m_{\tilde{g}}^{2} B_{1}^{\prime}\left(m_{\tilde{g}}^{2} ; m_{\tilde{g}}^{2}, 0\right)\right.} \\
& \left.+\sum_{\tilde{q}_{k}}\left\{B_{1}\left(m_{\tilde{g}}^{2} ; m_{q}^{2}, m_{\tilde{q}_{k}}^{2}\right)+2 m_{\tilde{g}}^{2} B_{1}^{\prime}\left(m_{\tilde{g}}^{2} ; m_{q}^{2}, m_{\tilde{q}}^{2}\right)\right\}\right] \\
\delta m_{\tilde{g}}=\frac{g_{s}^{2} m_{\tilde{g}}}{16 \pi^{2}}[ & \left.n_{c}-4 n_{c} B_{0}\left(m_{\tilde{g}}^{2} ; m_{\tilde{g}}^{2}, 0\right)-2 n_{c} B_{1}\left(m_{\tilde{g}}^{2} ; m_{\tilde{g}}^{2}, 0\right)-\sum_{\tilde{q}_{k}} B_{1}\left(m_{\tilde{g}}^{2} ; m_{q}^{2}, m_{\tilde{q}}^{2}\right)\right]
\end{aligned}
$$




\subsubsection{On-shell squark renormalisation}

The naive on-shell scheme. Using the standard OS formulas as presented in section 2.2.1 for deriving the wave-function renormalisation constants of the first and second generation squarks $\delta Z_{\tilde{q}}$ and third generation squarks $\delta Z_{\tilde{Q}}$, we obtain

$$
\begin{gathered}
\delta Z_{\tilde{q}}=\frac{g_{s}^{2} C_{F}}{8 \pi^{2}}\left[B_{0}\left(m_{\tilde{q}}^{2} ; m_{\tilde{q}}^{2}, 0\right)-B_{0}\left(m_{\tilde{q}}^{2} ; m_{\tilde{g}}^{2}, 0\right)+2 m_{\tilde{q}}^{2} B_{0}^{\prime}\left(m_{\tilde{q}}^{2} ; m_{\tilde{q}}^{2}, 0\right)+\left(m_{\tilde{g}}^{2}-m_{\tilde{q}}^{2}\right) B_{0}^{\prime}\left(m_{\tilde{q}}^{2} ; m_{\tilde{g}}^{2}, 0\right)\right], \\
\begin{array}{c}
\left(\delta Z_{\tilde{Q}}\right)_{i i}=\frac{g_{s}^{2} C_{F}}{8 \pi^{2}}[ \\
B_{0}\left(m_{\tilde{Q}_{i}}^{2} ; m_{\tilde{Q}_{i}}^{2}, 0\right)-B_{0}\left(m_{\tilde{Q}_{i}}^{2} ; m_{\tilde{g}}^{2}, m_{Q}^{2}\right)+2 m_{\tilde{Q}_{i}}^{2} B_{0}^{\prime}\left(m_{\tilde{Q}_{i}}^{2} ; m_{\tilde{Q}_{i}}^{2}, 0\right) \\
\left.+\left(m_{\tilde{g}}^{2}+m_{Q}^{2}-m_{\tilde{Q}_{i}}^{2}-4 m_{\tilde{g}} m_{Q} \Re\left\{\left(S_{\tilde{Q}}\right)_{i 1}^{*}\left(S_{\tilde{Q}}\right)_{i 2}\right\}\right) B_{0}^{\prime}\left(m_{\tilde{Q}_{i}}^{2} ; m_{\tilde{g}}^{2}, m_{Q}^{2}\right)\right], \\
\left(\delta Z_{\tilde{Q}}\right)_{i j}=-\frac{g_{s}^{2} C_{F} m_{\tilde{g}} m_{Q}}{4 \pi^{2}}\left(\left(S_{\tilde{Q}}\right)_{i 1}^{*}\left(S_{\tilde{Q}}\right)_{j 2}+\left(S_{\tilde{Q}}\right)_{i 2}^{*}\left(S_{\tilde{Q}}\right)_{j 1}\right) \frac{B_{0}\left(m_{\tilde{Q}_{j}}^{2} ; m_{Q}^{2}, m_{\tilde{g}}^{2}\right)-B_{0}\left(m_{\tilde{Q}_{i}}^{2} ; m_{Q}^{2}, m_{\tilde{g}}^{2}\right)}{m_{\tilde{Q}_{j}}^{2}-m_{\tilde{Q}_{i}}^{2}} .
\end{array}
\end{gathered}
$$

Similarly, the corresponding mass renormalisation constants read

$$
\begin{aligned}
\delta m_{\tilde{q}}^{2}= & \frac{g_{s}^{2} C_{F}}{8 \pi^{2}}\left[A_{0}\left(m_{\tilde{q}}^{2}\right)-A_{0}\left(m_{\tilde{g}}^{2}\right)-2 m_{\tilde{q}}^{2} B_{0}\left(m_{\tilde{q}}^{2} ; m_{\tilde{q}}^{2}, 0\right)+\left(m_{\tilde{q}}^{2}-m_{\tilde{g}}^{2}\right) B_{0}\left(m_{\tilde{q}}^{2} ; m_{\tilde{g}}^{2}, 0\right)\right], \\
\delta m_{\tilde{Q}_{i}}^{2}= & \frac{g_{s}^{2} C_{F}}{8 \pi^{2}}\left[\left(m_{\tilde{Q}_{i}}^{2}-m_{\tilde{g}}^{2}-m_{Q}^{2}+4 m_{\tilde{g}} m_{Q} \Re\left\{\left(S_{\tilde{Q}_{i}}\right)_{i 1}^{*}\left(S_{\tilde{Q}}\right)_{i 2}\right\}\right) B_{0}\left(m_{\tilde{Q}}^{2} ; m_{\tilde{g}}^{2}, m_{Q}^{2}\right)+\frac{A_{0}\left(m_{\tilde{Q}_{i}}^{2}\right)}{2}\right. \\
& \left.-A_{0}\left(m_{\tilde{g}}^{2}\right)-A_{0}\left(m_{Q}^{2}\right)-2 m_{\tilde{Q}_{i}}^{2} B_{0}\left(m_{\tilde{Q}_{i}}^{2} ; m_{\tilde{Q}_{i}}^{2}, 0\right)+\frac{1}{2} \sum_{k=1,2}\left\{\left|\left(S_{\tilde{Q}}\right)_{i k}\right|^{2} A_{0}\left(m_{\tilde{Q}_{k}}^{2}\right)\right\}\right],
\end{aligned}
$$

and the renormalisation of the top and bottom squark mixing angles is related to the one of their respective wave functions [72],

$$
\delta \theta_{\tilde{t}}=\frac{1}{4}\left[\delta Z_{\tilde{t}, 12}-\delta Z_{\tilde{t}, 21}^{*}\right] \quad \text { and } \quad \delta \theta_{\tilde{b}}=\frac{1}{4}\left[\delta Z_{\tilde{b}, 12}-\delta Z_{\tilde{b}, 21}^{*}\right] .
$$

This scheme however breaks weak interaction gauge invariance, as the physical squark masses are not allowed to be taken all independent. Left-handed up-type and down-type squarks of a given generation are indeed connected by $\mathrm{SU}(2)_{L}$ so that they cannot be renormalised independently. Such a scheme is however useful and valid for many phenomenological applications relying on simplified models inspired by the MSSM in which only a few particles and a subset of all MSSM Lagrangian terms are supplemented to the $\mathrm{SM}$, as for instance in the work of refs. $[73,74]$ or for the numerical results presented in the following sections of this paper. In the latter case, the relations between the physical squark masses are ignored as the relevant terms are not present in the simplified model Lagrangian, so that all fields can be renormalised independently. This approach however breaks down as soon as one considers an entire generation of squarks and wants to retain $\mathrm{SU}(2)_{L}$ gauge invariance as embedded in the MSSM.

In the rest of this subsection, we additionally present two of the most popular SUSY OS schemes, that will not be considered in our numerical simulation but that could easily 
be implemented in our framework as will be shown in section 2.3. Whilst the differences between all the possible schemes are formally of higher order, the corresponding higherorder contributions could be potentially large in some parts of the parameter space (for instance when $\tan \beta$ is large). Moreover, the different schemes necessitate different sets of input parameters, which becomes relevant for comparing their respective predictions.

The ' $m_{b}$ on-shell' scheme. As above-mentioned, gauge invariance under weak interactions implies that the masses of the left-handed up-type and down-type squarks of a given generation are connected to a unique bare soft mass parameter $\hat{\mathbf{m}}_{\tilde{\mathbf{Q}}}^{2}$. The tree-level squared mass matrices $\mathcal{M}_{\tilde{q}_{u}}^{2}$ and $\mathcal{M}_{\tilde{q}_{d}}^{2}$ associated with the up-type and down-type squarks of a given generation are indeed given, in the $\left(\tilde{q}_{L}, \tilde{q}_{R}\right)$ basis, by

$$
\begin{aligned}
& \mathcal{M}_{\tilde{q}_{u}}^{2}=\left(\begin{array}{cc}
\left(\hat{\mathbf{m}}_{\tilde{\mathbf{Q}}}^{2}\right)_{n n}+m_{q_{u}}^{2}+m_{Z}^{2} c_{2 \beta}\left[\frac{1}{2}-\frac{2}{3} s_{w}^{2}\right] & m_{q_{u}}\left(\left(\mathbf{A}^{\mathbf{u}}\right)_{n n}-\mu \cot \beta\right) \\
m_{q_{u}}\left(\left(\mathbf{A}^{\mathbf{u}}\right)_{n n}-\mu \cot \beta\right) & \left(\hat{\mathbf{m}}_{\tilde{\mathbf{U}}}^{2}\right)_{n n}+m_{q_{u}}^{2}+\frac{2}{3} m_{Z}^{2} c_{2 \beta} s_{w}^{2}
\end{array}\right), \\
& \mathcal{M}_{\tilde{q}_{d}}^{2}=\left(\begin{array}{cc}
\left(\hat{\mathbf{m}}_{\tilde{\mathbf{Q}}}^{2}\right)_{n n}+m_{q_{d}}^{2}-m_{Z}^{2} c_{2 \beta}\left[\frac{1}{2}-\frac{1}{3} s_{w}^{2}\right] & m_{q_{d}}\left(\left(\mathbf{A}^{\mathbf{d}}\right)_{n n}-\mu \tan \beta\right) \\
m_{q_{d}}\left(\left(\mathbf{A}^{\mathbf{d}}\right)_{n n}-\mu \tan \beta\right) & \left(\hat{\mathbf{m}}_{\tilde{\mathbf{D}}}^{2}\right)_{n n}+m_{q_{d}}^{2}-\frac{1}{3} m_{Z}^{2} c_{2 \beta} s_{w}^{2}
\end{array}\right),
\end{aligned}
$$

where $m_{Z}, c_{2 \beta}$ and $s_{w}$ stand for the mass of the $Z$-boson, $\cos 2 \beta$ and $\sin \theta_{w}$. We have furthermore indicated by a subscript $n$ the relevant generation index, and $m_{q_{u}}$ and $m_{q_{d}}$ are the masses of the corresponding up-type and down-type quarks $q_{u}$ and $q_{d}$. While for the first and second generations the off-diagonal terms vanish and those two matrices are diagonal, they must be further diagonalised for third generation squarks with the help of the two rotation matrices $S_{\tilde{t}}$ and $S_{\tilde{b}}$,

$$
\left(\begin{array}{cc}
m_{\tilde{t}_{1}}^{2} & 0 \\
0 & m_{\tilde{t}_{2}}^{2}
\end{array}\right)=S_{\tilde{t}} \mathcal{M}_{\tilde{t}}^{2} S_{\tilde{t}}^{\dagger} \quad \text { and } \quad\left(\begin{array}{cc}
m_{\tilde{b}_{1}}^{2} & 0 \\
0 & m_{\tilde{b}_{2}}^{2}
\end{array}\right)=S_{\tilde{b}} \mathcal{M}_{\tilde{b}}^{2} S_{\tilde{b}}^{\dagger} \text {. }
$$

Consequently, one of the four mass parameters associated with the first or the second generation of squarks is a dependent parameter and cannot be renormalised by imposing naive OS renormalisation conditions. Similarly, care must be taken with the stop/sbottom sector where we have six quark and squark masses $\left(m_{b}, m_{t}, m_{\tilde{t}_{1}}, m_{\tilde{t}_{2}}, m_{\tilde{b}_{1}}\right.$ and $\left.m_{\tilde{b}_{2}}\right)$, two mixing angles $\left(\theta_{\tilde{t}}\right.$ and $\left.\theta_{\tilde{b}}\right)$ as well as two soft trilinear interaction strengths $\left(A_{t} \equiv\left(\mathbf{A}^{\mathbf{u}}\right)_{33}\right.$ and $\left.A_{b} \equiv\left(\mathbf{A}^{\mathbf{d}}\right)_{33}\right)$. All these parameters are related and thus receive one-loop $\alpha_{S}$ corrections in a connected manner [75-78].

In the so-called ' $m_{b}$ on-shell' scheme, the renormalisation of the up-type and downtype squark sectors is performed simultaneously [79-81]. We consider the masses of the left-handed down and strange squarks as well as the one of the heaviest bottom squark as dependent parameters,

$$
\begin{aligned}
& m_{\tilde{d}_{L}}^{2}=m_{\tilde{u}_{L}}^{2}-m_{Z}^{2} c_{2 \beta} c_{w}^{2}, \quad m_{\tilde{s}_{L}}^{2}=m_{\tilde{c}_{L}}^{2}-m_{Z}^{2} c_{2 \beta} c_{w}^{2}, \\
& m_{\tilde{b}_{2}}^{2}=\frac{1}{\left|\left(S_{\tilde{b}}\right)_{12}\right|^{2}}\left[\left|\left(S_{\tilde{t}}\right)_{11}\right|^{2} m_{\tilde{t}_{1}}^{2}+\left|\left(S_{\tilde{t}}\right)_{21}\right|^{2} m_{\tilde{t}_{2}}^{2}-\left|\left(S_{\tilde{b}}\right)_{11}\right|^{2} m_{\tilde{b}_{1}}^{2}+m_{b}^{2}-m_{t}^{2}-m_{Z}^{2} c_{2 \beta} c_{w}^{2}\right],
\end{aligned}
$$


with $c_{w} \equiv \cos \theta_{w}$, so that the corresponding counterterms are given by

$$
\begin{aligned}
& \delta m_{\tilde{d}_{L}}^{2}=\delta m_{\tilde{u}_{L}}^{2}, \quad \delta m_{\tilde{s}_{L}}^{2}=\delta m_{\tilde{c}_{L}}^{2}, \\
& \delta m_{\tilde{b}_{2}}^{2}=\frac{1}{s_{\tilde{b}}^{2}}\left[c_{\tilde{t}}^{2} \delta m_{\tilde{t}_{1}}^{2}+s_{\tilde{t}}^{2} \delta m_{\tilde{t}_{2}}^{2}-c_{\tilde{b}}^{2} \delta m_{\tilde{b}_{1}}^{2}+2 m_{b} \delta m_{b}-2 m_{t} \delta m_{t}-s_{2 \tilde{t}} \Delta m_{\tilde{t}}^{2} \delta \theta_{\tilde{t}}+s_{2 \tilde{b}} \Delta m_{\tilde{b}}^{2} \delta \theta_{\tilde{b}}\right]
\end{aligned}
$$

where $\Delta m_{\tilde{t}}^{2}=m_{\tilde{t}_{1}}^{2}-m_{\tilde{t}_{2}}^{2}, \Delta m_{\tilde{b}}^{2}=m_{\tilde{b}_{1}}^{2}-m_{\tilde{b}_{2}}^{2}, s_{\tilde{t}, \tilde{b}} \equiv \sin \theta_{\tilde{t}, \tilde{b}}, s_{2 \tilde{t}, 2 \tilde{b}} \equiv \sin 2 \theta_{\tilde{t}, \tilde{b}}$ and $c_{\tilde{t}, \tilde{b}} \equiv \cos \theta_{\tilde{t}, \tilde{b}}$. We have explicitly introduced in those expressions the dependence on the mixing angles whose renormalisation constants $\delta \theta_{\tilde{t}}$ and $\delta \theta_{\tilde{b}}$ are given by eq. (2.36).

As a result, the renormalised masses of the left-handed down and strange squarks and of the heaviest bottom squarks are shifted with respect to their pole masses $m_{\tilde{d}_{L} \text {,pole' }}^{2}$, $m_{\tilde{s}_{L}, \text { pole }}^{2}$ and $m_{\tilde{b}_{2}, \text { pole }}^{2}[76]$,

$$
\begin{aligned}
m_{\tilde{d}_{L}, \text { pole }}^{2} & =m_{\tilde{d}_{L}}^{2}+\delta m_{\tilde{d}_{L}}^{2}-\delta m_{\tilde{d}_{L}, \text { pole }}^{2}, \quad m_{\tilde{s}_{L}, \text { pole }}^{2}=m_{\tilde{s}_{L}}^{2}+\delta m_{\tilde{s}_{L}}^{2}-\delta m_{\tilde{s}_{L}, \text { pole }}^{2}, \\
m_{\tilde{b}_{2}, \text { pole }}^{2} & =m_{\tilde{b}_{2}}^{2}+\delta m_{\tilde{b}_{2}}^{2}-\delta m_{\tilde{b}_{2}, \text { pole }}^{2}
\end{aligned}
$$

where $\delta m_{\tilde{d}_{L} \text {,pole }}, \delta m_{\tilde{s}_{L}, \text { pole }}$ and $\delta m_{\tilde{b}_{2} \text {,pole }}$ stand for the naive OS renormalisation constants of eq. (2.35) and $\delta m_{\tilde{d}_{L}}^{2}, \delta m_{\tilde{s}_{L}}^{2}$ and $\delta m_{\tilde{b}_{2}}^{2}$ are the ' $m_{b}$ on-shell' counterterms of eq. (2.40). The tree-level masses are moreover given by eq. (2.39). These UV-finite shifts must in particular be accounted for when an entire MSSM spectrum is used, as typical MSSM spectrum generators solely output pole squark masses.

By virtue of eq. (2.37) and eq. (2.38), the strengths of the soft trilinear squark-Higgs interactions $A_{t}$ and $A_{b}$ also receive one-loop corrections in $\alpha_{S}$ through their connection with the corresponding squark mixing angles,

$$
s_{2 \tilde{t}}=\frac{2 m_{t}\left(\mu \cot \beta-A_{t}\right)}{\Delta m_{\tilde{t}}^{2}} \quad \text { and } \quad s_{2 \tilde{b}}=\frac{2 m_{b}\left(\mu \tan \beta-A_{b}\right)}{\Delta m_{\tilde{b}}^{2}} .
$$

The corresponding counterterms are given by

$$
\delta A_{q}=\frac{1}{m_{q}}\left[\frac{1}{2}\left(\delta m_{\tilde{q}_{1}}^{2}-\delta m_{\tilde{q}_{2}}^{2}\right) s_{2 \tilde{q}}+\Delta m_{\tilde{q}}^{2} c_{2 \tilde{q}} \delta \theta_{\tilde{q}}-\frac{1}{2 m_{q}} \Delta m_{\tilde{q}}^{2} s_{2 \tilde{q}} \delta m_{q}\right] \quad \text { for } \quad q=b, t,
$$

as both the $\mu$ parameter and $\tan \beta$ do not receive $\alpha_{S}$ corrections at one loop.

The ' $\boldsymbol{A}_{\boldsymbol{b}} / \boldsymbol{\theta}_{\tilde{\boldsymbol{b}}}$ on-shell' scheme. As a consequence of eq. (2.43), two of the three counterterms $\delta m_{q}, \delta A_{q}$ and $\delta \theta_{\tilde{q}}$ are independent. There are thus various options for fixing the renormalisation conditions, that all lead to slight differences in the predictions. In the ' $m_{b}$ OS' scheme, the two $\delta A_{q}$ renormalisation constants are derived from the other counterterms. This is however known to yield potentially-unacceptably large threshold corrections to the bottom-quark pole mass due to the $\delta A_{b}$ counterterm when $\tan \beta$ is substantial $[80,82-84]$. Whilst a fully $\overline{\mathrm{DR}}$ renormalisation of the bottom sector $\left(\delta m_{b}, \delta A_{b}\right.$ and $\left.\delta \theta_{\tilde{b}}\right)$ would avoid the problem, this is also known not to make manifest the decoupling of heavy particles. 
We therefore present here another commonly-used scheme in which the $A_{b}$ parameter is renormalised in the OS scheme via a kinematic condition on the coupling of the pseudoscalar Higgs boson $A^{0}$ to a $\tilde{b}_{1} \tilde{b}_{2}$ pair. This approach relies on the proportionality of the $A^{0} \tilde{b}_{1} \tilde{b}_{2}$ coupling to the product of the bottom Yukawa coupling (or the bottom mass) and the bottom trilinear coupling, so that shifts in one quantity can always be reabsorbed in the other one. In practice, we calculate the one-loop corrections to the above-mentioned vertex with appropriately chosen external momenta and include suitable wave-function corrections to avoid any infrared divergence,

$$
\delta A_{b}=\frac{\left[A_{b} s_{\beta}+\mu c_{\beta}\right]\left[-\Delta m_{\tilde{b}}^{2} s_{2 \beta} \mathcal{F}\left(m_{\tilde{b}_{1}}^{2}, m_{\tilde{b}_{2}}^{2}\right)+\left(\delta m_{\tilde{b}_{1}}^{2}-\delta m_{\tilde{b}_{2}}^{2}\right) s_{2 \beta}+2 \Delta m_{\tilde{b}}^{2} c_{2 \beta} \delta \theta_{\tilde{b}}\right]}{2 m_{b} \mu c_{\beta}+s_{\beta}\left[2 A_{b} m_{b}-\Delta m_{\tilde{b}}^{2} s_{2 \beta}\right]} .
$$

In the above expression, the $\mathcal{F}$ function originates from the one-loop corrections to the $A^{0} \tilde{b}_{1} \tilde{b}_{2}$ vertex,

$$
\begin{aligned}
\mathcal{F}\left(m_{1}^{2}, m_{2}^{2}\right)=-\frac{g_{s}^{2} C_{F}}{8 \pi^{2}} & {\left[\frac{-m_{\tilde{g}}}{A_{b}+\mu \cot \beta}\left[B_{0}^{\mathrm{fin} .}\left(m_{1}^{2} ; m_{b}^{2}, m_{\tilde{g}}^{2}\right)+B_{0}^{\mathrm{fin} .}\left(m_{2}^{2} ; m_{b}^{2}, m_{\tilde{g}}^{2}\right)\right]\right.} \\
& +\frac{m_{1}^{2}}{m_{1}^{2}-m_{2}^{2}}\left(4+2 \log \frac{\mu_{R}^{2}}{m_{1}^{2}}-\frac{m_{1}^{2}-m_{\tilde{g}}^{2}-m_{b}^{2}}{m_{1}^{2}} B_{0}^{\mathrm{fin} .}\left(m_{1}^{2} ; m_{b}^{2}, m_{\tilde{g}}^{2}\right)\right) \\
& \left.+\frac{m_{2}^{2}}{m_{2}^{2}-m_{1}^{2}}\left(4+2 \log \frac{\mu_{R}^{2}}{m_{2}^{2}}-\frac{m_{2}^{2}-m_{\tilde{g}}^{2}-m_{b}^{2}}{m_{2}^{2}} B_{0}^{\text {fin. }}\left(m_{2}^{2} ; m_{b}^{2}, m_{\tilde{g}}^{2}\right)\right)\right],
\end{aligned}
$$

where only the finite pieces of the loop integrals are retained (i.e. all pieces independent of $1 / \bar{\epsilon}$ in the conventions of appendix A) and $\mu_{R}$ stands for the renormalisation/regularisation scale. As a consequence, the bottom mass counterterm is now a dependent parameter,

$$
\delta m_{b}=\frac{2 m_{b}}{\tan \beta} \delta \theta_{\tilde{b}}-\frac{2 m_{b}^{2}}{s_{2 \beta} \Delta m_{\tilde{b}}^{2}} \delta A_{b}+\frac{m_{b}}{\Delta m_{\tilde{b}}^{2}}\left(\delta m_{\tilde{b}_{1}}^{2}-\delta m_{\tilde{b}_{2}}^{2}\right) .
$$

\subsubsection{Renormalisation of the strong coupling}

Our calculations require that the running of the strong coupling constant $\alpha_{S}$ originates solely from the contributions of the gluons and $n_{l f}$ flavours of light quarks. We therefore renormalise the strong coupling by subtracting, at zero-momentum transfer, all massive particle contributions and $\overline{\mathrm{MS}}$ contributions of all massless particles from the gluon selfenergy [85-87]. They are then absorbed in the renormalisation constant of the strong coupling $\delta \alpha_{S}$ with $n_{l f}=4$,

$$
\begin{aligned}
\frac{\delta \alpha_{S}}{\alpha_{S}}= & \frac{\alpha_{S}}{2 \pi \bar{\epsilon}}\left[\frac{n_{l f}}{3}-\frac{11 n_{c}}{6}\right]+\frac{\alpha_{S}}{6 \pi}\left[\frac{1}{\bar{\epsilon}}-\log \frac{m_{b}^{2}}{\mu_{R}^{2}}\right]+\frac{\alpha_{S}}{6 \pi}\left[\frac{1}{\bar{\epsilon}}-\log \frac{m_{t}^{2}}{\mu_{R}^{2}}\right]+\frac{\alpha_{S} n_{c}}{6 \pi}\left[\frac{1}{\bar{\epsilon}}-\log \frac{m_{\tilde{g}}^{2}}{\mu_{R}^{2}}\right] \\
& +\frac{\alpha_{S}}{24 \pi} \sum_{\tilde{q}}\left[\frac{1}{\bar{\epsilon}}-\log \frac{m_{\tilde{q}}^{2}}{\mu_{R}^{2}}\right],
\end{aligned}
$$

where the sum in the last term includes all twelve squark species. The UV-divergent part of the renormalisation constant has been written explicitly in terms of the quantity

$$
\frac{1}{\bar{\epsilon}}=\frac{1}{\epsilon}-\gamma_{E}+\log 4 \pi,
$$


where $\gamma_{E}$ is the Euler-Mascheroni constant and $\epsilon$ is related to the number of space-time dimensions $D=4-2 \epsilon$.

The above renormalisation procedure however leads to a violation of SUSY as it introduces a mismatch between the strong coupling $g_{s}$ and the Yukawa interaction $\hat{g}_{s}$ of a gluino with a squark and a quark. While these two couplings are equal at tree-level, as shown by the last term of the second line of eq. (2.7), the equality is destroyed by the difference in the number of fermionic gluino degrees of freedom and bosonic gluon degrees of freedom. This artificial breaking of SUSY is compensated by finite counterterms restoring SUSY invariance.

As we impose that the definition of the strong coupling $g_{s}$ is the SM one due to the decoupling theorem, only the quark-squark-gluino vertices and quartic squark interactions have to be shifted [88]. The SUSY restoring counterterm Lagrangian $\mathcal{L}_{\mathrm{MSSM}}^{(\mathrm{SCT})}$ is then given, in the gauge eigenbasis, by

$$
\begin{aligned}
\mathcal{L}_{\mathrm{MSSM}}^{(\mathrm{SCT})}= & \frac{g_{s}^{2}}{2} \frac{\alpha_{s}}{4 \pi}\left[\tilde{q}_{R}^{\dagger}\left\{T_{a}, T_{b}\right\} \tilde{q}_{R}+\tilde{q}_{L}^{\dagger}\left\{T_{a}, T_{b}\right\} \tilde{q}_{L}\right]\left[\tilde{q}_{R}^{\dagger}\left\{T^{a}, T^{b}\right\} \tilde{q}_{R}+\tilde{q}_{L}^{\dagger}\left\{T^{a}, T^{b}\right\} \tilde{q}_{L}\right] \\
& -\frac{g_{s}^{2}}{2} \frac{\alpha_{s}}{4 \pi}\left[\tilde{q}_{R}^{\dagger} T_{a} \tilde{q}_{R}-\tilde{q}_{L}^{\dagger} T_{a} \tilde{q}_{L}\right]\left[\tilde{q}_{R}^{\dagger} T^{a} \tilde{q}_{R}-\tilde{q}_{L}^{\dagger} T^{a} \tilde{q}_{L}\right] \\
& +\sqrt{2} g_{s} \frac{\alpha_{s}}{3 \pi}\left[-\tilde{q}_{L}^{\dagger} T_{a}\left(\bar{\Psi}_{\tilde{g}}^{a} P_{L} \Psi_{q}\right)+\left(\bar{\Psi}_{q} P_{L} \Psi_{\tilde{g}}^{a}\right) T_{a} \tilde{q}_{R}+\text { h.c. }\right],
\end{aligned}
$$

where adjoint colour indices have been included and a sum over (s)quark flavours is understood for clarity.

\subsection{Technical details on the model implementation in FEYnRuleS}

In order to calculate SUSY particle-production (total and differential) rates at colliders and to simulate MSSM signals by matching fixed-order results at the NLO accuracy with parton showers, we rely on the MG5_AMC framework [56]. Our methodology is based on the joint usage of the FeynRules [58], NLOCT [59] and FeynARTs [89] packages to automatically produce a UFO model [57] that can be used by MG5_AMC. However, there are substantial differences with respect to the procedure that has been followed for stop pair production [74], in the SUSY QCD case [73] and for slepton production [90], as a consequence of the non-trivial renormalisation procedure for the mixing angle and the trilinear scalar couplings detailed in section 2.2.4. After having implemented the model described in section 2.1 and its tree-level Lagrangian in terms of superfields, we make use of the superspace module of FeYnRuLEs [91] to re-express the MSSM Lagrangian in terms of the model physical degrees of freedom and four-component fermions. The renormalisation is then performed with the MoGRE package, that is introduced in appendix B and that is necessary for a flexible definition of the renormalisation scheme.

We firstly impose that all external parameters insensitive to QCD corrections are kept unrenormalised. We hence enforce vanishing renormalisation constants for all electroweak inputs (the Fermi constant $G_{F}$, the inverse of the electromagnetic coupling at the $Z$ pole $1 / \alpha$ and the $Z$-boson mass $\left.m_{Z}\right)$, the parameters of the Higgs sector $(\tan \beta$, the $\alpha$ angle and the $\mu$ parameter), the slepton trilinear couplings $\left(A_{e}, A_{\mu}\right.$ and $\left.A_{\tau}\right)$, the soft masses 
associated with the electroweak particles and the electroweakino mixing matrices $(U, V$ and $N$ ) that are external parameters in the SLHA conventions [65]. Moreover, the first and second generation squark trilinear couplings $\left(A_{u}, A_{d}, A_{c}\right.$ and $\left.A_{s}\right)$ are irrelevant as multiplied by a vanishing quark mass and will thus not be renormalised. These constraints are imposed by using the MoGRe`DefineUnrenormalizedParameter function introduced in appendix B.2.

Secondly, the quark mass dependence of the (remaining) trilinear squark-Higgs couplings $\left(\hat{\mathbf{T}}^{\mathbf{u}}\right.$ and $\hat{\mathbf{T}}^{\mathbf{d}}$ ) as well as the one of the fermion Yukawa couplings must be made explicit to guarantee the correct functioning of NLOCT. This is achieved by making use of the RemovingInternalCst method introduced in appendix B.6.1. The same method is finally also used to replace all occurrences of the $g_{s}$ renormalisation constant in terms of the $\alpha_{S}$ one.

Next, we indicate to the code that fields that are insensitive to the strong interaction at the one-loop level (the electroweak gauge and Higgs bosons) do not need to be renormalised. This is achieved by making use of the MoGRe`DefineUnrenormalizedField method detailed in appendix B.2. Whilst other purely electroweak fields such as electroweakinos or (s)leptons have in principle to be analogously tagged as unrenormalisable objects, they do not appear in any QCD vertex so that they will be automatically discarded by the code. We finally impose that all field wave-function renormalisation constants are real (via the MoGRe`RealFieldRenormalization method presented in appendix B.2).

In practice, the MoGRE package is initialised as

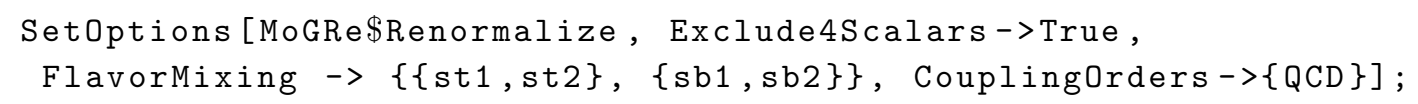

which indicates to the code that the stop and sbottom fields mix and that the renormalisation of the four-scalar interactions can be ignored. While strictly speaking, four-scalar interactions cannot be ignored, restrictions omitting them are useful phenomenologically as these vertices rarely appear at tree-level. The constraints above-mentioned are then implemented as follows,

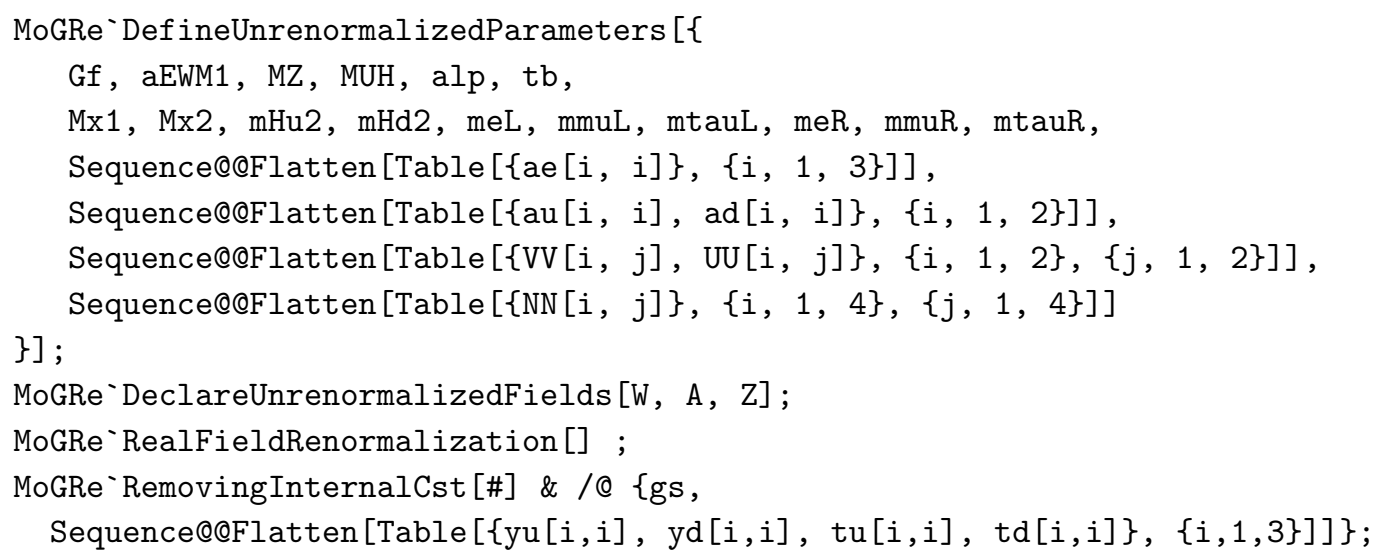

The exact details of the renormalisation scheme must then be specified, as shown in appendix B.4. Focusing on the naive OS scheme, the stop and sbottom mixing matrices are renormalised on the basis of eq. (2.36), which is implemented as 
MoGRe`AddRenormalizationCondition [FR $\$ \operatorname{delta}[\{\operatorname{Rtop}[1,1]\},\{\}], 1 / 4$ Rtop $[2,1]$ * (FR\$deltaZ[\{st1, st2\}, $\{\{\}\}]-$ Conjugate $[F R \$ d e l t a Z[\{$ st2, st1\}, $\{\{\}\}]])] ;$ MoGRe`AddRenormalizationCondition [FR $\$$ delta [\{Rtop [1, 2]\}, \{\}], 1/4 Rtop [1, 1]* (FR\$deltaZ[\{st1, st2\}, $\{\{\}\}]$ - Conjugate[FR $\$$ deltaZ[\{st2, st1\}, $\{\{\}\}]])]$; MoGRe`AddRenormalizationCondition [FR $\$$ delta [\{Rtop [2, 1]\}, \{\}], 1/4 Rtop [1, 1]* (FR\$deltaZ[\{st1, st2\}, $\{\{\}\}]$ - Conjugate [FR\$deltaZ[\{st2, st1\}, $\{\{\}\}]])]$; MoGRe`AddRenormalizationCondition [FR\$delta[\{Rtop [2, 2]\}, \{\}], 1/4 Rtop [2, 1]* (FR\$deltaZ[\{st1, st2\}, $\{\{\}\}]-$ Conjugate $[F R \$ d e l t a Z[\{$ st2, st1\}, $\{\{\}\}]])] ;$ MoGRe`AddRenormalizationCondition [FR $\$$ delta $[\{\operatorname{Rbot}[1,1]\},\{\}], 1 / 4 \operatorname{Rbot}[2,1] *$ (FR\$deltaZ $[\{\mathrm{sb} 1, \operatorname{sb} 2\},\{\{\}\}]-$ Conjugate $[\mathrm{FR} \$ \operatorname{deltaZ}[\{\mathrm{sb} 2, \operatorname{sb} 1\},\{\{\}\}]])] ;$ MoGRe`AddRenormalizationCondition [FR $\$$ delta[\{Rbot $[1,2]\},\{\}], 1 / 4 \operatorname{Rbot}[1,1] * i$ (FR\$deltaZ[\{sb1, sb2\}, \{\{\}\}] - Conjugate [FR\$deltaZ[\{sb2, sb1\}, \{\{\}\}]])]; MoGRe`AddRenormalizationCondition [FR $\$ d \operatorname{delta}[\{\operatorname{Rbot}[2,1]\},\{\}], 1 / 4 \operatorname{Rbot}[1,1] *$ (FR\$deltaZ $[\{\mathrm{sb} 1, \operatorname{sb} 2\},\{\{\}\}]-$ Conjugate $[\mathrm{FR} \$ \operatorname{deltaZ}[\{\mathrm{sb} 2, \operatorname{sb} 1\},\{\{\}\}]])] ;$ MoGRe`AddRenormalizationCondition [FR $\$ \operatorname{delta}[\{\operatorname{Rbot}[2,2]\},\{\}], 1 / 4 \operatorname{Rbot}[2,1] *$ (FR\$deltaZ[\{sb1, sb2\}, \{\{\}\}] - Conjugate $[F R \$ d e l t a Z[\{s b 2, \operatorname{sb} 1\},\{\{\}\}]])] ;$

the stop and sbottom mixing matrices $S_{\tilde{t}}$ and $S_{\tilde{b}}$ being available as the parameters Rtop and Rbot in the FeynRules implementation. The renormalisation of the stop and sbottom sector is finalised by imposing the way in which the $A_{t}$ and $A_{b}$ parameters are renormalised, according to eq. (2.43),

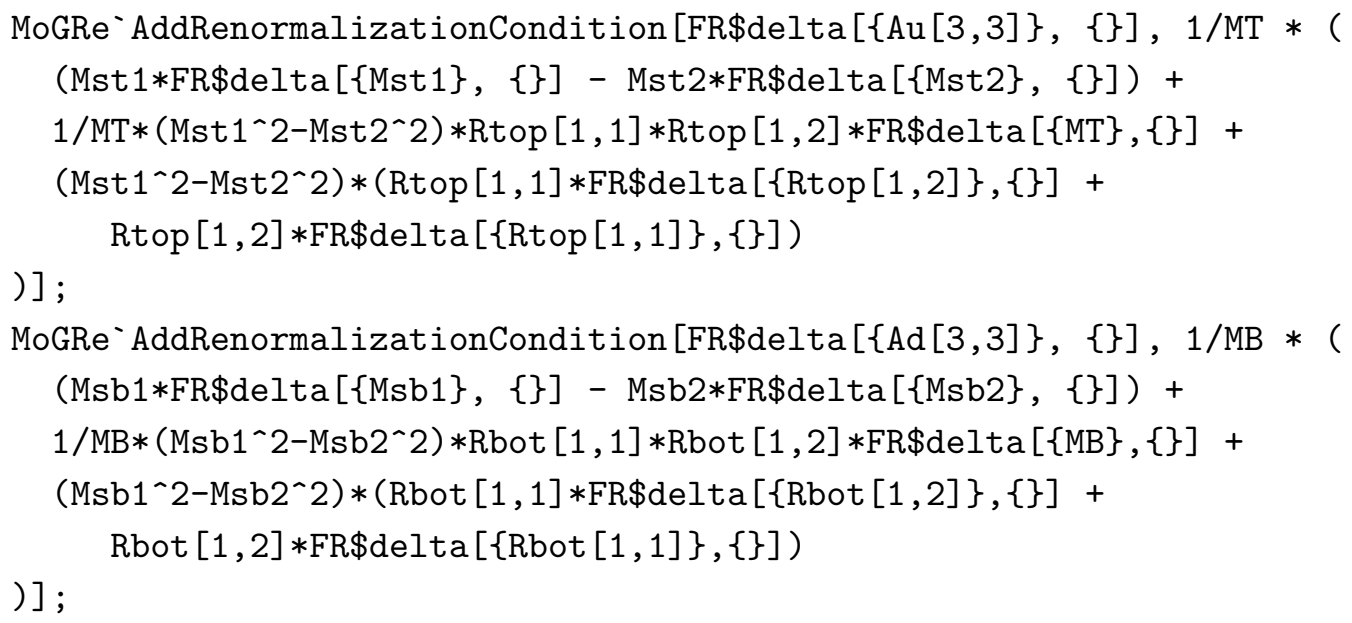

We subsequently make use of NLOCT to generate the ultraviolet counterterms and $R_{2}$ Feynman rules necessary to obtain, from the UFO interface of FeYnRules, an NLO UFO model for the MSSM. As the calculations performed by NLOCT rely on dimensional regularisation, as the predictions obtained with MG5_AMC in the following sections, one needs to compensate the artificial breaking of supersymmetry induced by the mismatch of the two gluino and $(D-2)$ gluon degrees of freedom. We therefore add to the model Lagrangian a set of finite counterterms allowing to restore supersymmetry at the one-loop level. Enforcing the definition of $g_{s}$ to be the SM one, quark-squark-gluino and four-scalar interactions are the only interactions that need to be shifted. Those shifts are given by the 
following counterterm Lagrangian [88],

$$
\begin{aligned}
\mathcal{L}_{\text {shift }}= & \sqrt{2} g_{s} \frac{\alpha_{s}}{3 \pi}\left[-\tilde{q}_{L}^{\dagger} T_{a}\left(\bar{\Psi}_{\tilde{g}}^{a} P_{L} \Psi_{q}\right)+\left(\bar{\Psi}_{q} P_{L} \Psi_{\tilde{g}}^{a}\right) T_{a} \tilde{q}_{R}+\text { h.c. }\right] \\
& -\frac{g_{s}^{2}}{2} \frac{\alpha_{s}}{4 \pi}\left[\tilde{q}_{R}^{\dagger} T_{a} \tilde{q}_{R}-\tilde{q}_{L}^{\dagger} T_{a} \tilde{q}_{L}\right]\left[\tilde{q}_{R}^{\dagger} T^{a} \tilde{q}_{R}-\tilde{q}_{L}^{\dagger} T^{a} \tilde{q}_{L}\right] \\
& +\frac{g_{s}^{2}}{2} \frac{\alpha_{s}}{4 \pi}\left[\tilde{q}_{R}^{\dagger}\left\{T_{a}, T_{b}\right\} \tilde{q}_{R}+\tilde{q}_{L}^{\dagger}\left\{T_{a}, T_{b}\right\} \tilde{q}_{L}\right]\left[\tilde{q}_{R}^{\dagger}\left\{T^{a}, T^{b}\right\} \tilde{q}_{R}+\tilde{q}_{L}^{\dagger}\left\{T^{a}, T^{b}\right\} \tilde{q}_{L}\right],
\end{aligned}
$$

where $\mathcal{L}_{\text {shift }}$ is written in the gauge eigenbasis. Those counterterms are appropriately included in the MSSM UFO in two steps [74]. We first evaluate the associated Feynman rules with FEYNRULES and then provide the resulting set of rules to the UFO interface by means of the UVLoopCounterterms option of the WriteUFO method.

In contrast to the previous approaches, the resulting model can be used, within MG5_AMC, beyond the simplified model context. We have verified that the resulting UFO model was consistent with all the analytical results presented in this section, taking a particular care of the numerous minus signs and factors of $i$ inherent to SUSY theories.

\subsection{Simulations and cross section calculations in SUSY}

As was discussed in section 1, it is common practice to normalise the results of tree-level merged simulations by means of higher-order inclusive cross sections. NLO+NLL total production rates are known for light-flavour squarks [92-100], third-generation squarks [101103], gluinos [93, 95-100, 104], electroweakinos [105-109], sleptons [105, 110-113], and for several mixed channels involving one strong and one electroweak superpartner [114, 115]. In addition, NLO QCD corrections have been computed including superparticle decays for squark pair-production $[116,117],{ }^{1}$ while approximate NNLO threshold contributions $[118$ 120], (electro)weak (EW) corrections [81, 121-129], and resummation effects at the NNLL level [130-136] have been considered for squark and gluino production. Moreover, effects originating from $R$-parity violation $[137,138]$ and non-minimal flavour-violation [139141] have also been explored. All of these results have been included in several publicly available computer programs, which are restricted to the evaluation of total rates. Prospino2 [93, 101, 105] and MadGolem [142-144] give predictions that are NLO-QCD accurate, while NLL-FAST [145], NNLL-FAst [146] and Resummino [147] also resum threshold logarithms. All codes, with the exception of MadGolem and Resummino, assume mass-degenerate squark spectra.

Theoretical predictions at the differential level, that include both NLO and PS effects, are more recent and, so far, tackled on a process-by-process basis. The production of pairs of squarks [73, 74, 148, 149], gluinos [74], electroweakinos [150, 151], and sleptons $[90,152,153]$ have all been considered in the past few years. Some of these computations have been carried out with MG5_AMC. We recall here that MG5_AMC makes use of the FKS method [154, 155] (automated in the module MADFKS $[156,157]$ ) for dealing with IR singularities. The computations of one-loop amplitudes are carried out by switching dynamically between two integral-reduction techniques, OPP [158] or Laurent-series

\footnotetext{
${ }^{1}$ Both the $2 \rightarrow 2$ matrix element describing the production process and the $1 \rightarrow 2$ matrix elements related to the decay processes are NLO-QCD accurate, the different contributions being assumed to factorise as it is the case in the narrow-width approximation.
} 
expansion [159], and tensor-integral reduction [71, 160, 161]. These have been automated in the module MAdLoop [56, 162], which in turn exploits CutTools [163], Ninja [164, 165], IREGi [166], or ColLier [167], together with an in-house implementation of the OpenLoops optimisation [168]. Finally, in the case of matching with PS, the MC@NLO formalism [34] is employed, whereas NLO multi-jet mergings rely either on FxFx [50] or UNLOPS [53].

We point out that the original MG5_AMC paper [56] had the goal of including as much information on Quantum Field Theories as possible in the meta-code, so as to allow it to simulate both SM and BSM processes by using the inputs in the form of UFO models constructed by codes such as FeynRules or SARAh [169]. Recently, the program has been upgraded, and can for instance now handle mixed-coupling scenarios, in particular QCD+EW simultaneous corrections [70]. However, the most general BSM calculations beyond LO feature a number of non-trivial characteristics that are absent in the SM. While fermion-flow-violating interaction vertices and non-renormalisable operators (which were not available at the time of the first release [56]) can now be handled, coloured-sextet particles and the renormalisation-group running of new couplings are not yet included in MG5_AMC. Thus, we stress again that the current work will be limited to considering NLO QCD corrections to SUSY theories, whereby only quarks, gluons, squarks, and gluinos can run in the loops. Moreover, real-emission contributions only consider additional massless SM particles in the final state, given that massive particle contributions are finite (and can thus be computed independently) and often numerically subleading (see the analogous discussion of ref. [70] that addresses Heavy Boson Radiation (HBR) in the context of the computation of NLO electroweak corrections).

We conclude this section by listing the UFO models that can presently be used for BSM simulations. These include simplified models, in which the SM is extended by colour-triplet and octet scalar particles [73, 170], both gluinos and squarks [74] or sleptons [90], as well as by vector-like quarks [171, 172], a heavy top-philic scalar [173] or a spin-2 particle [174]. In the latter spin-2 case, new physics has also been previously explored in a semi-automated framework (in the sense where the virtual matrix elements are provided externally) based on MG5_AMC [175-177]. Various implementations of BSM setups in which the Higgs sector differs from the SM one have been released, including the two-Higgs-doublet model [178, 179], the Georgi-Machacek model [180], the Higgs characterisation model [181-185], and the SM effective field theory including dimension-six operators [186]. Higher-dimension operators either affecting the sector of the top quark [187-192], dijet production [193], or $Z$-boson production [194] can be added as well. Moreover, the model library also allows for NLO+PS calculations in BSM models involving $\mathrm{TeV}$-scale neutrinos [195], a left-right symmetry [196], as well as extra neutral and charged gauge bosons [197]. Finally, dark matter simplified models in which the dark matter particle is produced in $s$-channels are also available [198-205].

As was stressed in section 1, part of the present paper is devoted to creating a UFO model of the MSSM, which is still missing in an unrestricted framework (i.e. when going beyond the simplified-model approach). The lifting of such a restriction has also to do with the treatment of resonant contributions, also addressed here through the STR procedures. 


\section{$3 \quad$ Validation}

Fixed-order NLO-QCD predictions for the total rates of specific two-to-two processes in the MSSM are currently available from three different standalone tools, namely ProsPINO2 [93, 101, 105], Resummino [147], and MadGolem [142-144]. A partial comparison of the results obtained with MG5_AMC and MADGOLEM has already been performed for coloured-scalar production, and agreement at the level of the numerical errors has been found [73]. Furthermore, the analytic expressions of all the $R_{2}$ counterterms of the MSSM model have been cross-checked against the results of ref. [206]. In this section, we employ MG5_AMC to compute total rates for several processes and specific choices of the MSSM parameters, and compare our predictions against those obtained with Prospino2 (that covers the production of any pair of strongly- or electroweakly-interacting superpartners in the case of a degenerate squark mass spectrum) and RESUMmino (that supports arbitrary SUSY mass spectra for the production of two electroweak superpartners). In the rest of this paper, all fermions are unambiguously four-component Dirac and Majorana ones, so that we replace the notation $\Psi_{X}$ by $X$. In particular, quarks, gluino and electroweakinos will be denoted by $q, \tilde{g}$, and $\tilde{\chi}$, respectively.

\subsection{Setup of the comparison}

Although we shall focus on superparticle-pair production here, it should be clear that, in keeping with a general automation philosophy, MG5_AMC is not restricted to simulating processes with two-body final states. Furthermore, MG5_AMC lifts two other key limitations of current NLO QCD codes: firstly, the inability to tackle QCD-mediated production processes (REsummino); and secondly, the inability to support without approximation arbitrary (non-degenerate) squark mass spectra (Prospino2). In order to highlight these differences, we have opted to present a comparison of the cross sections, at the $13 \mathrm{TeV}$ LHC, relevant to the following processes (where antisquarks are denoted with a star):

$$
p p \longrightarrow \tilde{t}_{1} \tilde{t}_{1}^{\star}, \quad p p \longrightarrow \tilde{g} \tilde{g}, \quad p p \longrightarrow \tilde{\chi}_{1}^{+} \tilde{\chi}_{1}^{-}, \quad p p \longrightarrow \tilde{e}_{R}^{+} \tilde{e}_{R}^{-},
$$

in both regimes of degenerate and non-degenerate squark masses. We point out that we have explicitly checked that $\tilde{\chi}_{1}^{+} \tilde{\chi}_{2}^{0}$ and $\tilde{e}_{L}^{+} \tilde{e}_{L}^{-}$production lead to the same qualitative conclusions as $\tilde{\chi}_{1}^{+} \tilde{\chi}_{1}^{-}$and $\tilde{e}_{R}^{+} \tilde{e}_{R}^{-}$production, respectively; such final states are thus ignored in what follows. We have not considered a direct point-wise comparison of one-loop SUSY QCD amplitudes against those of FEYNARTs [207, 208], as the focus of our work is on the computation of cross sections and observables.

The model parameters corresponding to the considered SUSY benchmark point are specified in all three codes via a similar SLHA file [65], the contents of which are summarised in table 1. The complicated nature of the Prospino2 inputs prompted our use of two different sets of parton distribution functions (PDFs) for LO and NLO predictions, for which the appropriate value of $\alpha_{S}\left(m_{Z}^{2}\right)$ (equal to 0.08991 and 0.08314 , respectively) had to be hard-coded: by default, Prospino2 uses a fixed value of $\alpha_{S}\left(m_{Z}^{2}\right)$ independent of the PDF set. We have used the LO and NLO central sets of the CTEQ6 PDFs [209], and additionally turned off the running of $\alpha_{S}$. Moreover, whilst Prospino2 keeps the exact 


\begin{tabular}{|ll|ll|ll|}
\hline Parameter & value & Parameter & value & Parameter & value \\
\hline LO PDF set & cteq611 & $\mu_{R}=\mu_{F}$ & 1500 & $m_{t}$ & 174.3 \\
NLO PDF set & cteq66 & $m_{Z}$ & 91.188 & $m_{b}$ & 0 \\
$\alpha_{S}\left(m_{Z}^{2}\right)$ & as per PDF set & $G_{F}$ & $1.16637 \cdot 10^{-5}$ & $\Gamma_{\text {all particles }}$ & 0 \\
$\alpha$ & $1 / 127.9$ & SUSY-mixing & Only between $\tilde{\chi}_{1}^{0}-\tilde{\chi}_{2}^{0}$ & $\left(V_{\text {CKM }}\right)_{i j}$ & $\delta_{i j}$ \\
\hline \multicolumn{7}{|c|}{ Degenerate SUSY mass setup } \\
\hline$M_{\text {prod }}$ & 1500 & $M_{\text {others }}[100-1400]$ & $m_{\tilde{u}_{L}}$ & $M_{\text {others }}$ \\
\hline \multicolumn{7}{|c|}{ Non-degenerate SUSY mass setup } \\
\hline$M_{\text {prod }}$ & 1500 & $M_{\text {others }}$ & {$[100-1400]$} & $m_{\tilde{u}_{L}}$ & 1400 \\
\hline
\end{tabular}

Table 1. SM and SUSY parameters of the benchmark point used for the comparisons performed in section 3. Dimensionful quantities are given in $\mathrm{GeV}\left(\mathrm{GeV}^{-2}\right.$ for $\left.G_{F}\right) . M_{\text {prod }}$ denotes the mass of the produced particles in the processes of eq. (3.1), while $M_{\text {others }}$ denote the masses of all other SUSY particles in a degenerate mass setup (this quantity is scanned over, hence its range value). In the non-degenerate mass setup, the left-handed up squark mass is set equal to a fixed value of $1.4 \mathrm{TeV}$.

dependence on the masses of the produced sparticles both at the LO and the NLO, the masses that appear in all of the internal squark propagators are set equal to some averaged value when working at the NLO. The $K$-factor, defined as the ratio of such an "averaged" NLO computation over the corresponding LO one, is then used to multiply the exact LO result to get the final (and hence, approximate) NLO prediction. In order to assess the quality of such an approximation, we have scanned the cross sections obtained with all three codes in the two different mass setups defined in table 1.

In the case of a spectrum with degenerate SUSY masses, the mass of the produced SUSY particle $\left(M_{\text {prod }}\right)$ is set equal to $1.5 \mathrm{TeV}$, while the common mass of all other SUSY particles $\left(M_{\text {others }}\right)$ is scanned over, in the range $[100,1400] \mathrm{GeV}$. This insures that all of the masses that appear in internal propagators are equal to each other (i.e. the internal squarks are degenerate), with the possible exception of the propagators that involve the produced particles. For instance, $\tilde{t}_{1} \tilde{t}_{1}^{\star}$ production has diagrams with internal $\tilde{t}_{1}$ propagators: the corresponding mass is then kept equal to $1.5 \mathrm{TeV}$. In the case of a spectrum featuring non-degenerate SUSY masses, we use exactly the same configuration as for the degenerate case, except that this time the left-handed up squark mass $\left(m_{\tilde{u}_{L}}\right)$ is set equal $1.4 \mathrm{TeV}$. This particular choice for breaking the degeneracy pattern allows for an increase of the sensitivity of the inclusive cross section to the mass splitting $m_{\tilde{u}_{L}}-M_{\text {others }}$.

We stress that it is crucial to set the mass of the produced particles $M_{\text {prod }}$ to a value larger than all of the other masses, in order to insure the absence of any resonant realemission contributions (see section 5). Such contributions would in fact complicate the comparison among the three codes, which adopt different strategies for handling them, leading in turn to potential non-negligible differences in their predictions.

\subsection{Degenerate SUSY masses}

We report in figure 1 the outcome of the comparison among the LO and NLO predictions of MG5_AMC, Prospino2, and Resummino, for a degenerate SUSY mass setup and the 


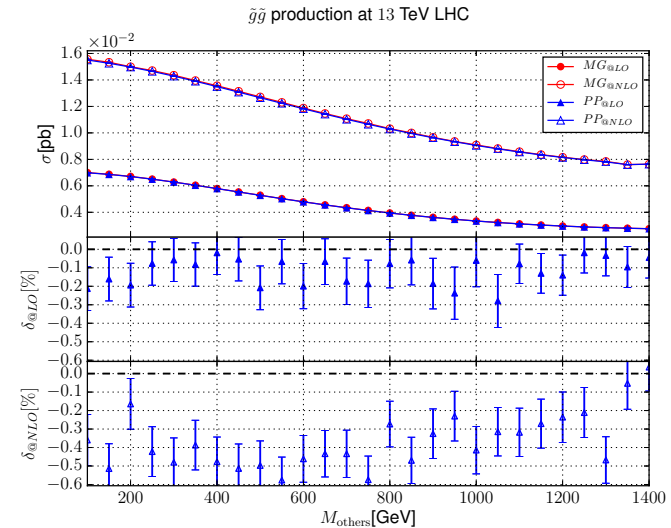

$\tilde{\chi}_{1}^{+} \tilde{\chi}_{1}^{-}$production at 13 TeV LHC

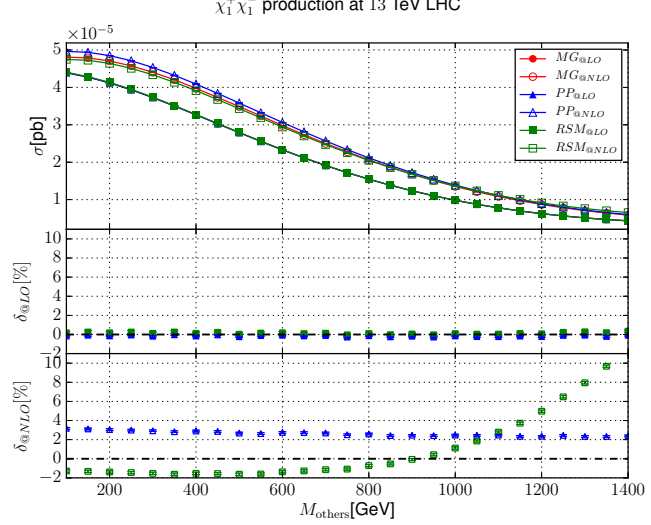

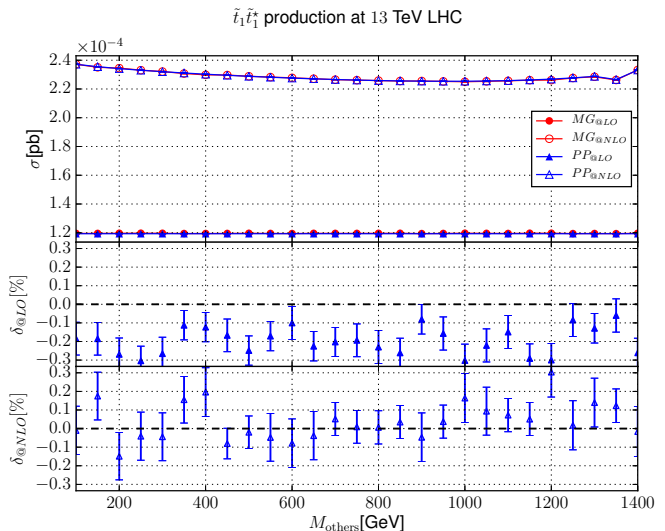

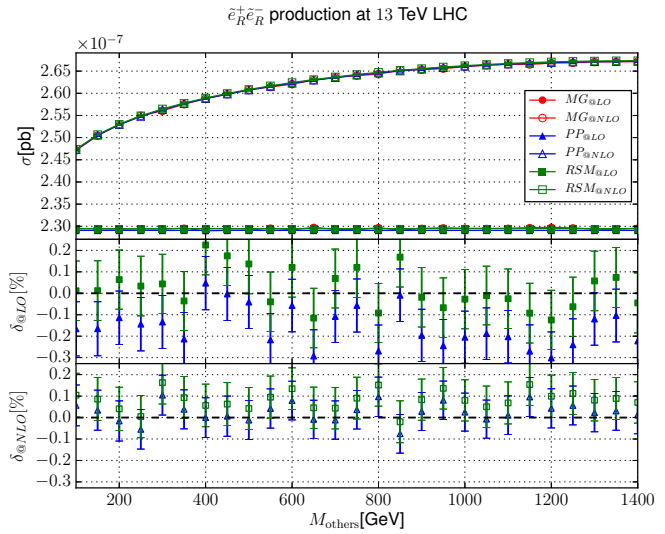

Figure 1. Comparison of inclusive LO (full symbols) and NLO (open symbols) cross sections obtained from MG5_AMC (red, MG), Prospino2 (blue, PP), and REsummino (green, RSM) for $\tilde{g} \tilde{g}$ (top left panel), $\tilde{\chi}_{1}^{+} \tilde{\chi}_{1}^{-}$(bottom left panel), $\tilde{t}_{1} \tilde{t}_{1}^{\star}$ (top right panel), and $\tilde{e}_{R}^{+} \tilde{e}_{R}^{-}$(bottom right panel) production at the $\sqrt{S}=13 \mathrm{TeV}$ LHC for degenerate squark masses. The two lower insets of each plot show the difference $\delta$ (in percent) relative to the MG5_AMC predictions, at the LO and the NLO. The width of the bands corresponds to the Monte-Carlo uncertainty of the predictions.

processes of eq. (3.1). As expected, we find no dependence on $M_{\text {others }}$ for inclusive stop (top right panel) and slepton (bottom right panel) pair production cross section at the LO, by virtue of the absence of internal SUSY particles of different flavours in the corresponding four-point tree-level amplitudes. This contrasts with the production of a pair of gluinos (top left panel) and charginos (bottom left panel), which both feature production modes that involve $t$-channel exchanges of squarks with different flavours.

The enhancement with respect to the LO results due to higher-order corrections is very significant for QCD-mediated processes $(\mathcal{O}(100 \%))$, and considerably milder for the electroweak processes $(\mathcal{O}(10 \%))$. One striking feature of the $M_{\text {others }}$ dependence of the NLO cross sections for both $\tilde{t}_{1} \tilde{t}_{1}^{\star}$ and $\tilde{g} \tilde{g}$ production lies in the characteristic kink appearing at $M_{\text {others }} \simeq 1330 \mathrm{GeV}$, which originates from the "resonant" anomalous thresholds [210] featured by the one-loop vertex corrections shown in figure 2. The latter cross a threshold at $M_{\text {others }}=M_{\text {prod }}-m_{t}$, that is also highlighted in the zoomed-in figure presented in the left panel of figure 2 . 

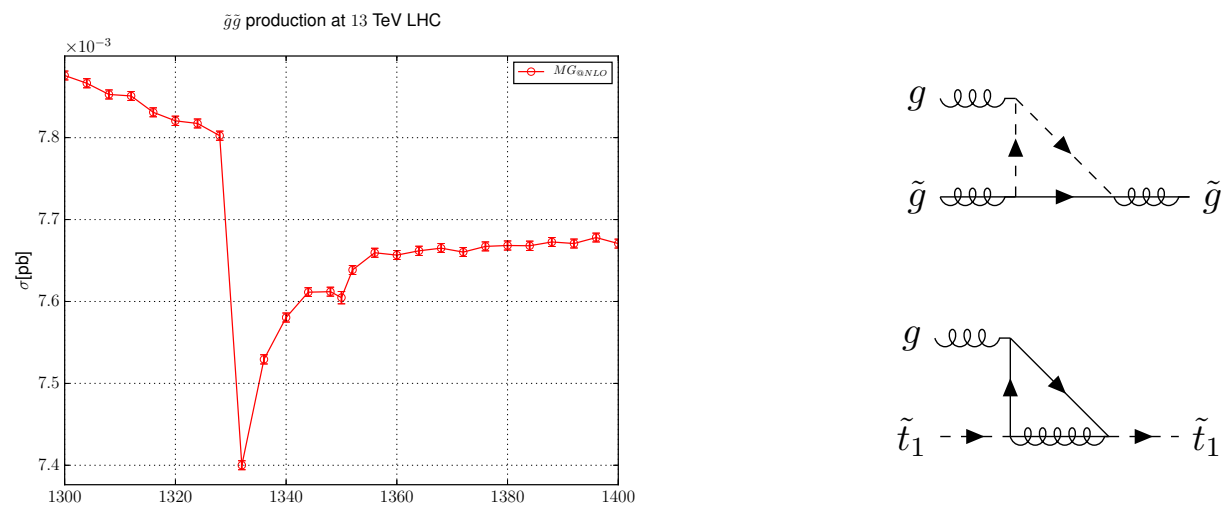

Figure 2. Left: MG5_AMC predictions for gluino pair-production at the $13 \mathrm{TeV}$ LHC, for $M_{\text {others }} \in[1300,1400] \mathrm{GeV}$. Right: vertex correction diagrams featuring an anomalous threshold at $M_{\text {others }}=M_{\text {prod }}-m_{t}$.

In the degenerate mass regime, one expects to find complete agreement among the three codes, which is what figure 1 basically shows at both the LO and the NLO, except in the case of chargino pair-production at the NLO, where large differences can be seen between any two predictions. We point out that, for the other processes, the agreement between MG5_AMC and Prospino2 is at the level of $0.5 \%$ at the worst (the latter cross sections being larger than the former ones). This may originate from a small mismatch in the input parameters, whose settings are especially intricate in PROSPINO2 as many of them are directly hard-coded in different parts of its source code (the value of $\alpha_{S}\left(m_{Z}^{2}\right)$ is a prime example of this fact). At the NLO, the Prospino2 results for chargino pairproduction also differ by a rather flat offset of 3\% relative to the MG5_AMC results. This might again be due to a mismatch in the input parameters, which however we could not track down. Conversely, for chargino production the shape of the dependence on $M_{\text {others }}$ of RESUmmino predictions is significantly different from the MG5_AMC one, and a preliminary investigation of the RESUMmino code has revealed issues in its SUSY-induced renormalisation of a specific vertex; this will be addressed in an upcoming release of the program by its authors. Given the discrepancies found for this process, we have carried out a fully independent analytic computation of the cut-constructible parts of the corresponding virtual matrix elements, and found perfect point-wise agreement with the corresponding automated numerical computations performed by MADLOOP.

\subsection{Non-degenerate SUSY masses}

Even in the absence of the issues we have outlined in section 3.2, in the non-degenerate squark regime we do not expect to find complete agreement between MG5_AMC and Prospino2. Therefore, at least for the processes for which we found have agreement in the degenerate scenario, we can assess the quality of the mass-averaging procedure implemented in Prospino2 to derive the NLO cross sections when the spectrum is non-degenerate. Figure 3 shows that this approximation can lead to differences with respect to the exact results (as computed by MG5_AMC) of several percent. The exception is stop-pair production, 


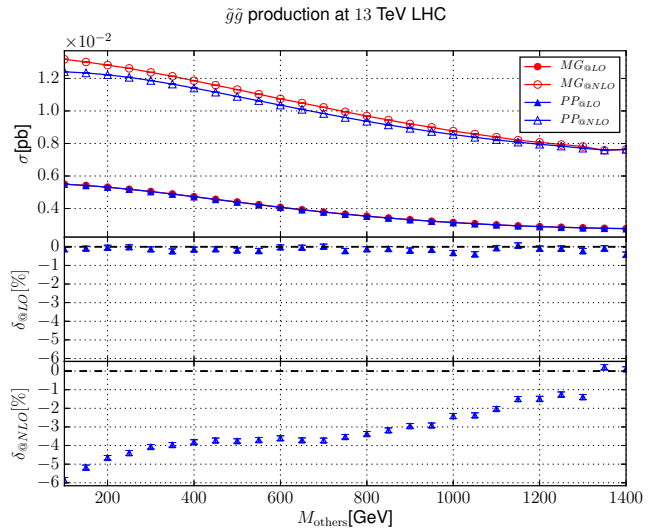

$\tilde{\chi}_{1}^{+} \tilde{\chi}_{1}^{-}$production at 13 TeV LHC

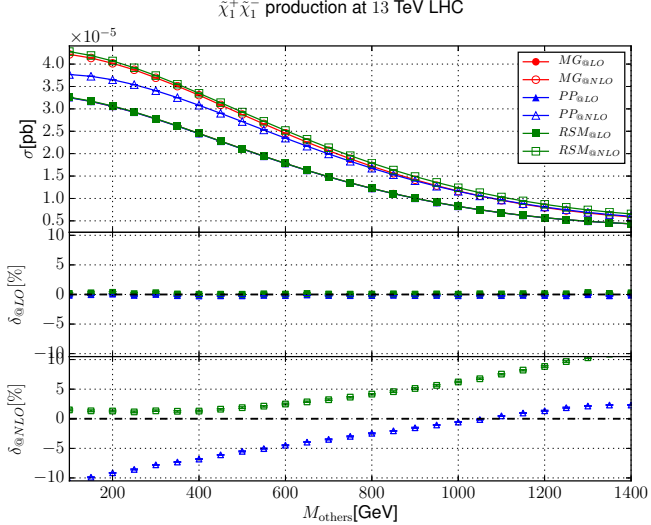

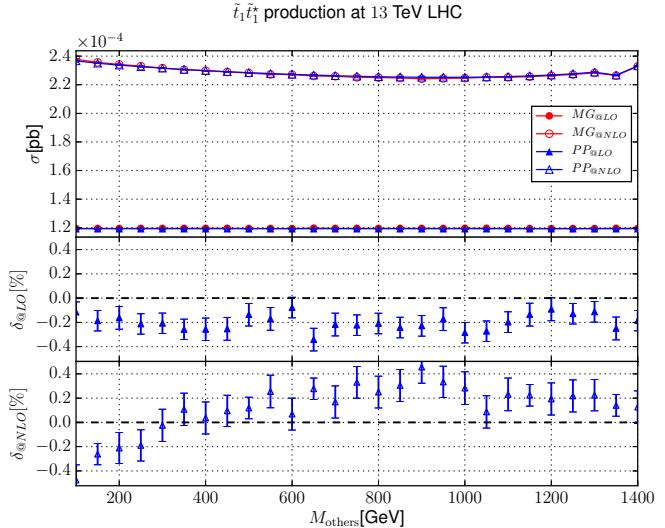

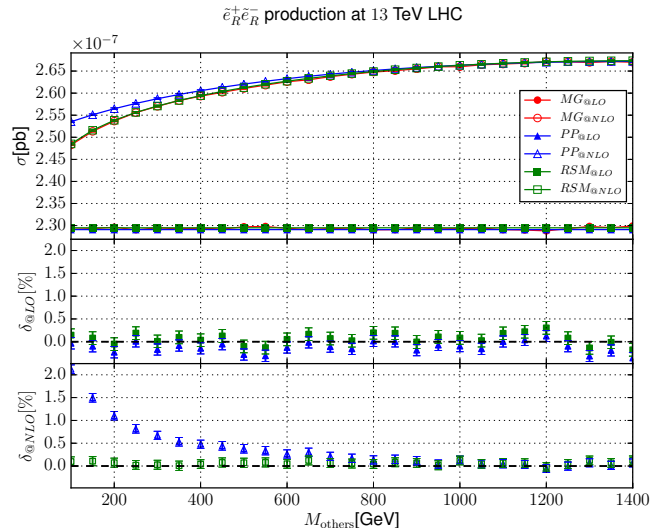

Figure 3. Same as figure 1, but for non-degenerate squark masses.

where the effect of the SUSY masses being non-degenerate is small, as they appear only in the virtual amplitudes (and not at the level of real-emission diagrams). As far as RESUMMINO is concerned, this program has been designed to deal with the dependence on arbitrary SUSY mass spectra in an exact manner. In spite of this, we do not find agreement when comparing its predictions with those of MG5_AMC for chargino-pair production, whilst the agreement in the slepton-pair production case has only been found after a couple of bug fixes in Resummino (that have been implemented in version 2.0.2; unfortunately, these do not address the issue with charginos, which is still under investigation).

\subsection{Summary of the comparisons}

The comparisons presented in this section, and the sometimes significant disagreement found among MG5_AMC, Prospino2, and Resummino results, underscore the need for a more comprehensive and robust implementation of NLO QCD corrections for SUSY processes, that can be reliably used by collider experiments. We believe that this is what is achieved currently by MG5_AMC, thanks to its highly automated approach, and its history of orthogonal cross-checks from applications and validation of the same framework to other models and simulations. 


\section{Total rates for supersymmetric benchmark processes in simplified sce- narios}

In this section we calculate the total cross sections at the NLO in QCD for several supersymmetric processes in the context of simplified models, that are typically employed for the interpretation of SUSY searches at the LHC. In these scenarios, one assumes that only the final-state SUSY particles are relatively light, while all of the other superpartners are decoupled by their very large masses. This setup allows us to avoid to deal with intermediate resonances in the real-emission contributions, which will be extensively discussed in section 5 .

\subsection{Setup of the calculation}

We consider the processes $p p \rightarrow \tilde{X} \tilde{Y}$ (where we denote by $\tilde{X}$ and $\tilde{Y}$ two SUSY particles, which may also be identical) at the $\sqrt{S}=13 \mathrm{TeV}$ LHC, at its high-energy upgrade with $\sqrt{S}=27 \mathrm{TeV}$, and at a potential future proton-proton collider, identified as the FCC-hh, with $\sqrt{S}=100 \mathrm{TeV}$. If $\tilde{X}$ and $\tilde{Y}$ are of different species, we enforce their masses to be equal, $m_{\tilde{X}}=m_{\tilde{Y}}$. All of the other SUSY particles which do not appear in the final states are decoupled by setting their masses equal to $15 \mathrm{TeV}(30 \mathrm{TeV}, 110 \mathrm{TeV})$ when $\sqrt{S}=13 \mathrm{TeV}$ $(27 \mathrm{TeV}, 100 \mathrm{TeV}$, respectively), with the exception of the two stop states whose masses are fixed to $m_{\tilde{t}_{1}}=16 \mathrm{TeV}(32 \mathrm{TeV}, 120 \mathrm{TeV})$ and $m_{\tilde{t}_{2}}=17 \mathrm{TeV}(34 \mathrm{TeV}, 130 \mathrm{TeV})$. We refer to appendix $\mathrm{C}$ for details on the complexity of a numerical implementation of the decoupling of heavy SUSY particles. The widths of particles are taken to be equal to zero, and the central values of the factorisation and renormalisation scales are set as follows:

$$
\mu_{F}=\mu_{R} \equiv \tilde{M}=\frac{m_{\tilde{X}}+m_{\tilde{Y}}}{2} .
$$

The theoretical uncertainty stemming from the missing higher-order corrections is estimated by a nine-point independent scale variation, $\left(\mu_{F}, \mu_{R}\right)=\left(\xi_{F}, \xi_{R}\right) \tilde{M}$, with $\xi_{F / R}=1 / 2$, 1, 2. We use the NNPDF30_nlo_as_0118 set of parton densities [211] as provided by the LHAPDF6 interface [212].

\subsection{Production of a pair of SUSY particles of the same species}

In this section, we consider six pair-production processes in which $\tilde{X}$ and $\tilde{Y}$ are of the same species. NLO total rates are shown in the left panel of figure 4 , for $\sqrt{S}=13 \mathrm{TeV}$ proton-proton collisions resulting in the production of a pair of gluinos ( $\tilde{g} \tilde{g}$, black circles), a pair of light stops $\left(\tilde{t}_{1} \tilde{t}_{1}^{\star}\right.$, red diamonds), a pair of left-handed up squarks $\left(\tilde{u}_{L} \tilde{u}_{L}^{\star}\right.$, yellow triangles), a pair of left-handed and right-handed selectrons $\left(\tilde{e}_{L}^{+} \tilde{e}_{L}^{-}\right.$and $\tilde{e}_{R}^{+} \tilde{e}_{R}^{-}$, blue circles and brown triangles, respectively), and a pair of (opposite-sign) charginos $\left(\tilde{\chi}_{1}^{+} \tilde{\chi}_{1}^{-}\right.$, green triangles). In order to improve the visibility of the different curves, we have included a rescaling factor equal to 0.1 in the case of $\tilde{u}_{L} \tilde{u}_{L}^{\star}$ production. Our results include the bands associated with the theoretical uncertainties obtained from the independent variations of the renormalisation and factorisation scales, as well as from the PDF uncertainties; these are added in quadrature. 

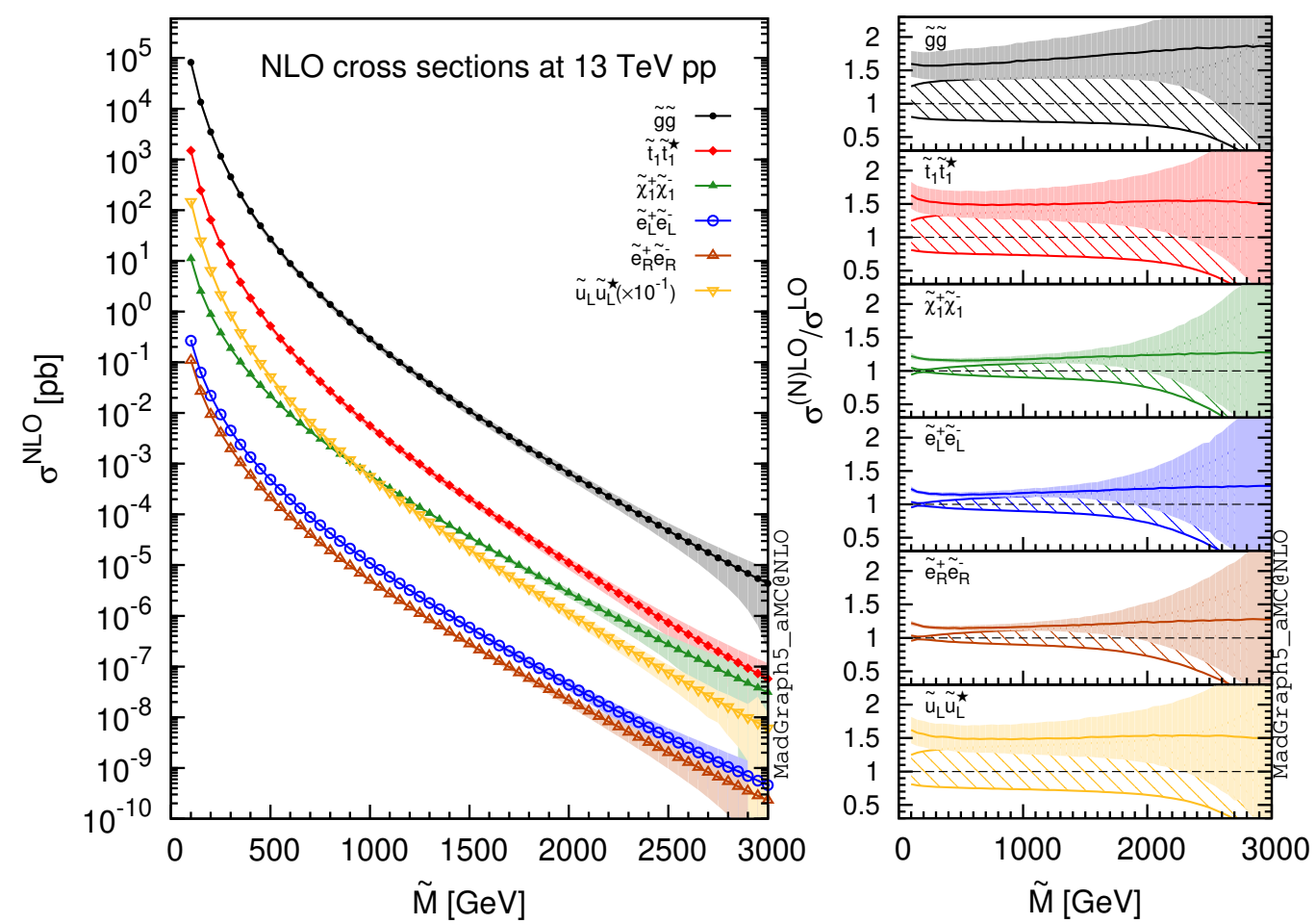

Figure 4. Total NLO cross sections (left) and $K$-factors defined as the ratio of the NLO result to the corresponding LO one (right) for the six processes associated with the production of a pair of SUSY particles in the same species at $\sqrt{S}=13 \mathrm{TeV}$ LHC. The different bands correspond to the sum of the scale and PDF uncertainties in quadrature, the NLO ones being indicated by filled areas and the LO ones (shown on the right panel) by hashed areas.

The NLO cross sections are found to span about 10 orders of magnitude when the SUSY mass $\tilde{M}$ varies from $100 \mathrm{GeV}$ to $3 \mathrm{TeV}$, with the strong production of squark and gluino pairs being larger than the electroweak production of slepton or electroweakino pairs by orders of magnitude, for any given $\tilde{M}$ value. We also show the associated $K$ factors in the right panel of the figure, where each $K$-factor is defined as the ratio of the NLO total rate over the corresponding LO one evaluated at the central scale and with the central set of PDF. The depicted uncertainty therefore reflects the standard NLO cross section uncertainty, as extracted relative to the central LO predictions. The $K$-factors exhibit different behaviours for the different processes. Firstly, they are larger in the cases of strong squark and gluino production $(K \sim 1.5)$ than for Drell-Yan-like slepton and chargino production $(K \sim 1.2)$, as is expected from the strong/electroweak nature of such processes. Secondly, the $K$-factor associated with the $\tilde{g} \tilde{g}$ process shows a significant dependence on the SUSY mass $\tilde{M}$, which can be traced back to the virtual amplitudes associated with the quark-antiquark contribution to the cross section and the large gluino colour charge [93]. Whilst subdominant for small SUSY masses, the quarkantiquark contribution becomes significant when $\tilde{M}$ increases (since the relative weight of the corresponding parton luminosity increases with respect to the $g g$ one), and it therefore impacts the cross section to a more significant level. Conversely, the $\tilde{M}$ dependence of the 
two $K$-factors associated with the production of a pair of squarks is more moderate, and almost absent in the case of the electroweak processes.

The right panel of figure 4 illustrates the benefits of higher-order calculations, as it shows that theoretical systematics are smaller at the NLO (filled areas) than at the LO (hashed areas). However, predictions relevant to large $\tilde{M}$ values are affected, both at the LO and the NLO, by significant uncertainties. This is because, in this region, the latter are dominated by PDF errors. In fact, by increasing $\tilde{M}$, the average Bjorken $x$ 's that enter the partonic cross sections also grow, and at large $x$ 's the PDFs are poorly constrained. Fortunately, one expects that a stronger constraining power of the searches for SUSY signals will go hand in hand with better-quality data for SM processes at large scales, which in turn will help to reduce the PDF uncertainties.

Similar results as in figure 4 can be found when the centre-of-mass energy is set equal to $\sqrt{S}=27 \mathrm{TeV}$ (see figure 5) and $100 \mathrm{TeV}$ (see figure 6). As is expected, the main difference with respect to the $13 \mathrm{TeV}$ case is the increase of the cross sections at any given $\tilde{M}$, due to the larger available centre-of-mass energies. By scaling up the SUSY mass $\tilde{M}$ to match the collider energy, the behaviours of the $K$-factors are essentially identical for the three collider scenarios.

\subsection{Production of a pair of SUSY particles of different species}

We now consider the production of two SUSY particles of different species, while still setting their masses equal to a common value $\tilde{M}$. In figure 7 , we present the dependence of the NLO cross sections on $\tilde{M}$ for nine different SUSY pair-production processes, in proton-proton collisions at $\sqrt{S}=13 \mathrm{TeV}$. We focus on two strong processes in which a gluino is produced in association with a left-handed up squark $\left(\tilde{g} \tilde{u}_{L}\right.$, red diamonds) or antisquark ( $\tilde{g} \tilde{u}_{L}^{\star}$, green triangles), as well as four semi-strong processes corresponding to the production of a gluino and a neutralino $\left(\tilde{g} \chi_{1}^{0}\right.$, black circles), a gluino and a chargino $\left(\tilde{g} \chi_{1}^{+}\right.$, brown triangles), a left-handed up squark and a neutralino $\left(\tilde{u}_{L} \chi_{1}^{0}\right.$, yellow triangles $)$ and a left-handed up squark and a chargino $\left(\tilde{u}_{L} \chi_{1}^{-}\right.$, magenta squares $)$. Our results finally also include predictions for three electroweakino pair-production processes in which the lightest chargino is produced in association with the lightest neutralino $\left(\tilde{\chi}_{1}^{+} \tilde{\chi}_{1}^{0}\right.$, turquoise squares) or with the next-to-lightest neutralino $\left(\tilde{\chi}_{1}^{+} \tilde{\chi}_{2}^{0}\right.$ and $\tilde{\chi}_{1}^{-} \tilde{\chi}_{2}^{0}$, purple diamonds and red pentagons). For all of our predictions, the lightest neutralino is taken to be bino-like, whilst the next-to-lightest neutralino and the lightest chargino are both taken to be winolike. Analogously to what has been done previously, we present the corresponding $K$-factors on the right panel of figure 7 , and we include theoretical errors estimated by summing in quadrature the uncertainties stemming from scale variations and the PDF errors.

Gluino-squark cross sections (i.e. with a $\tilde{g} \tilde{u}_{L}$ or a $\tilde{g} \tilde{u}_{R}$ final state) are identical to each other, these processes being driven by strong interactions that are blind with respect to the (s)quark chirality. Owing to the larger up-quark density in the proton with respect to the antiup-quark one, the corresponding conjugate processes are suppressed by factors that range from a few units (for small SUSY masses) to almost two orders of magnitude (for large SUSY masses). This is illustrated in figure 7 for $\tilde{g} \tilde{u}_{L}^{\star}$ production (the cross section for $\tilde{g} \tilde{u}_{R}^{\star}$ is identical to the latter one, and is not shown). The $K$-factors associated with all these 

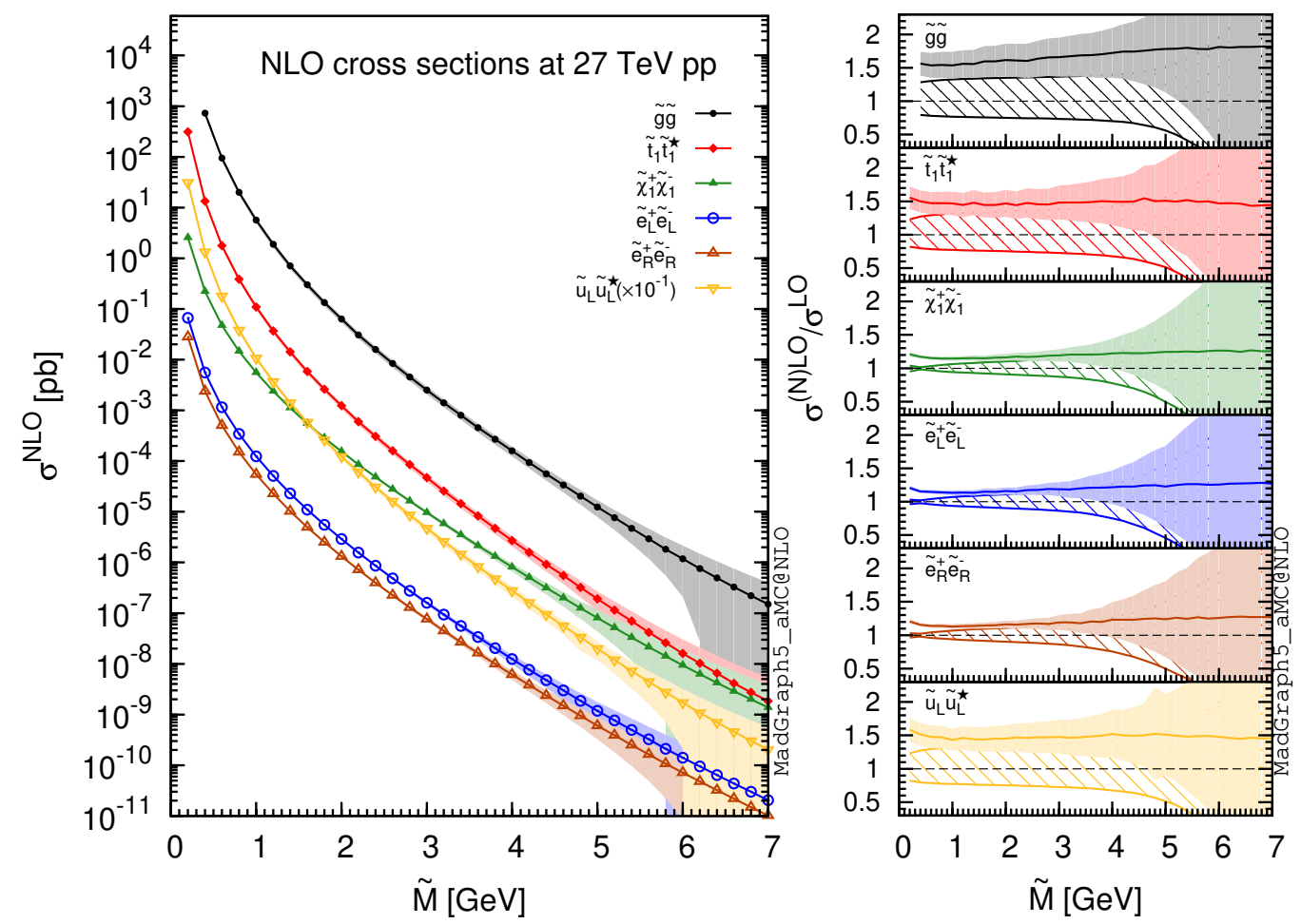

Figure 5. Same as figure 4, but for proton-proton collisions at $\sqrt{S}=27 \mathrm{TeV}$.
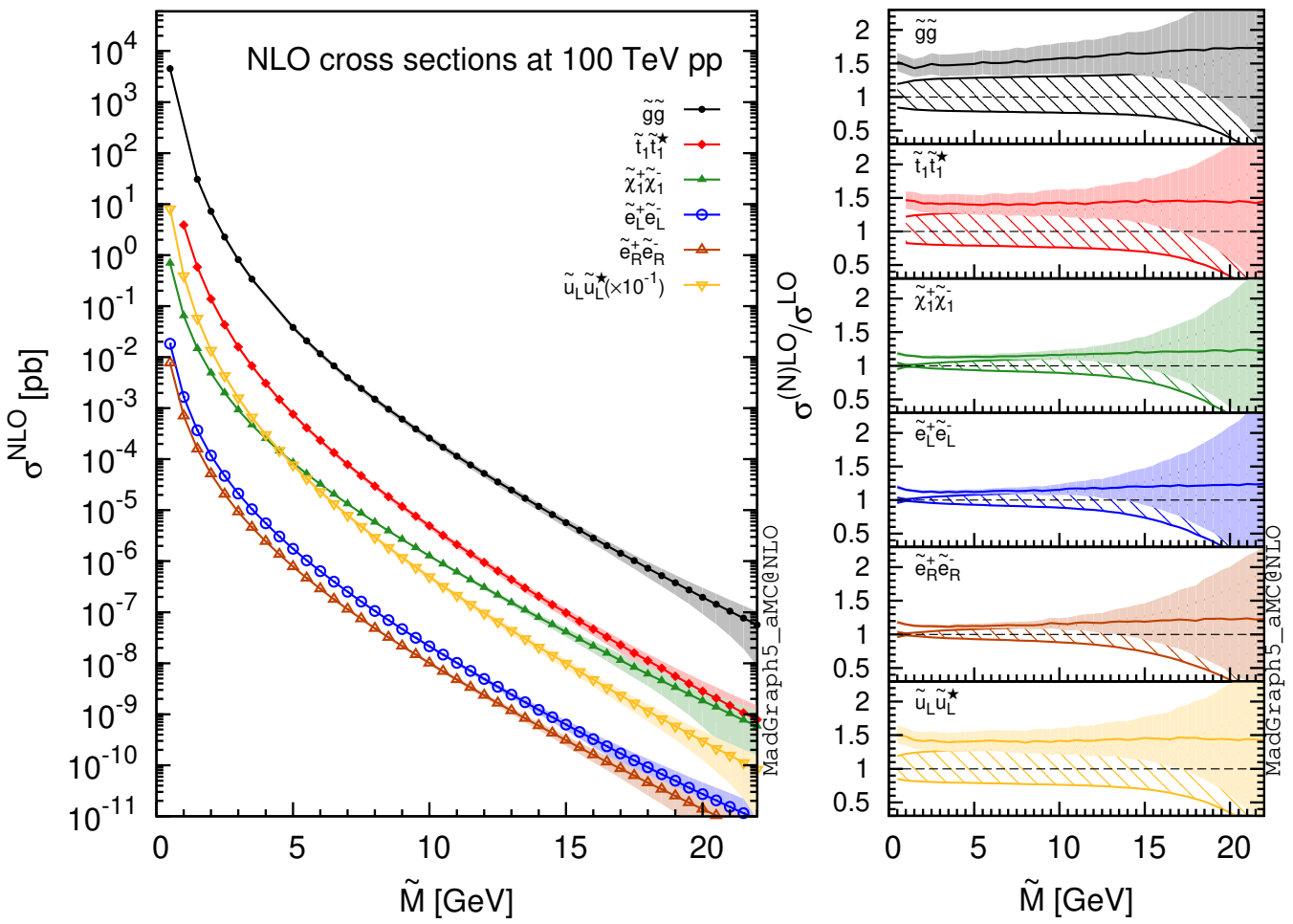

Figure 6. Same as figure 4 , but for proton-proton collisions at $\sqrt{S}=100 \mathrm{TeV}$. 

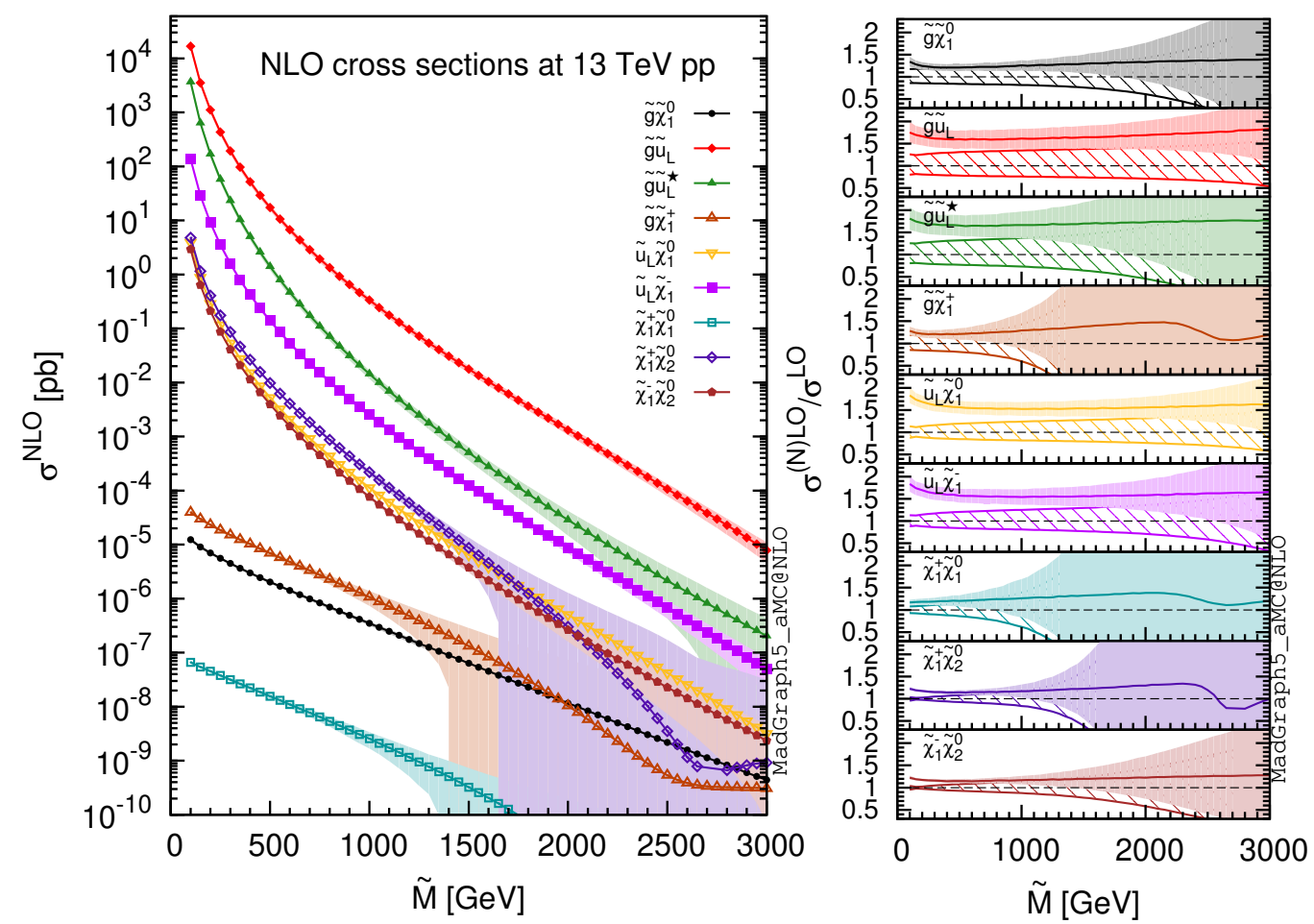

Figure 7. Total NLO cross sections (left) and $K$-factors (right) for nine processes involving the production of an associated pair of different SUSY particles with identical mass $\tilde{M}$ at $\sqrt{S}=13 \mathrm{TeV}$ LHC. The different bands correspond to the quadratic sum of the scale and PDF uncertainties, the NLO ones being indicated by filled areas and the LO ones (shown on the right panel) by hashed areas.

strong processes depend significantly on the SUSY mass $\tilde{M}$, and vary from about 1.5-1.6 for small $\tilde{M}$ 's to about 1.9 for multi-TeV $\tilde{M}$. As was already observed in section 4.2 , it is the gluino with its large associated colour charge that drives this dependence of the NLO $\mathrm{K}$ factors. Theoretical systematics follow the same pattern as those relevant to same-species production, namely scale uncertainties are reduced at the NLO, whilst the total uncertainty increases for large $\tilde{M}$ because of the PDF behaviour. This is especially significant in the case of the antiquark density, so that we accordingly find a larger uncertainty for $\tilde{g} \tilde{u}_{L}^{\star}$ production than for $\tilde{g} \tilde{u}_{L}$ production.

The other processes under consideration are of either purely electroweak or semi strong/electroweak nature. The rates are consequently reduced by several orders of magnitude, and the $K$-factors turn out to be smaller than for the purely strong processes. As is shown in the right panel of figure 7, QCD corrections are in general larger for the semi-strong processes featuring a gluino or a squark in the final state $(K \sim 1.5)$, as is expected from their sensitivity to strong interactions that is present already at the tree level. Conversely, the purely-electroweak electroweakino pair production processes exhibit smaller $K$-factors of about 1.2, a typical value for the Drell-Yan-like electroweakino pairproduction that occurs when all squarks are decoupled. The mass dependences of the $K$-factors are moreover modest, with the exception of the peculiar behaviour exhibited 


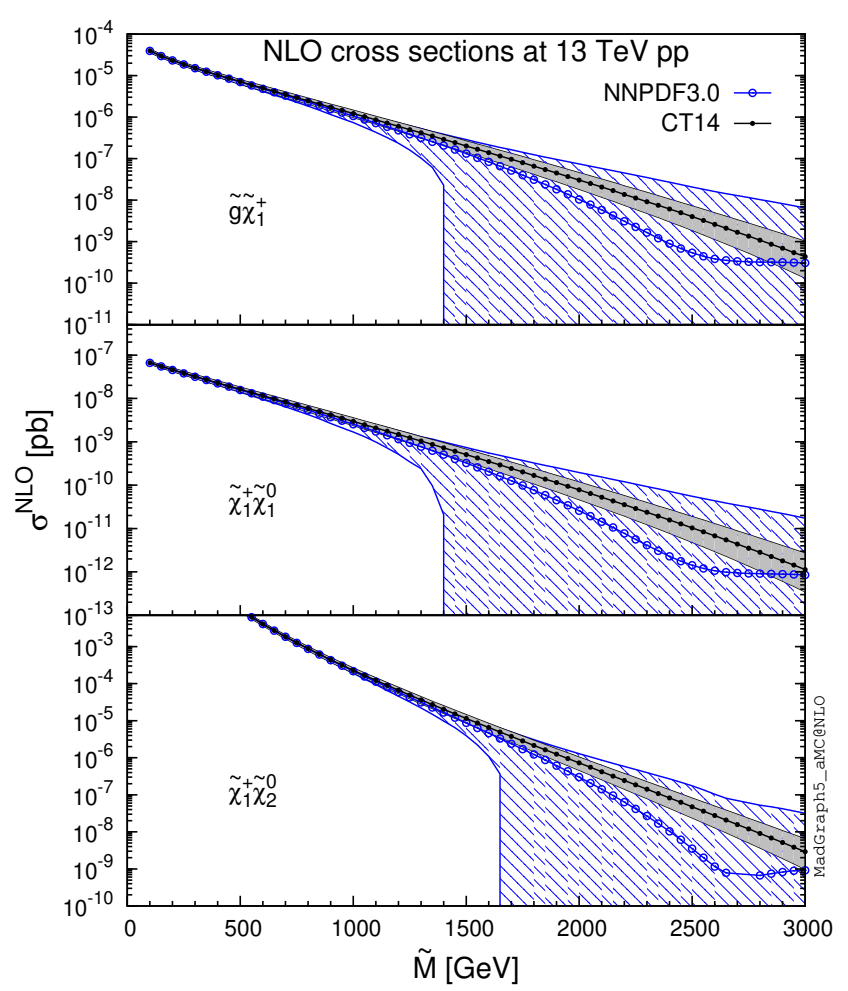

Figure 8. Total NLO cross sections for $\tilde{g} \tilde{\chi}_{1}^{+}$(upper panel), $\tilde{\chi}_{1}^{+} \tilde{\chi}_{1}^{0}$ (middle panel) and $\tilde{\chi}_{1}^{+} \tilde{\chi}_{2}^{0}$ (lower panel) production. We compare the results obtained when the NLO matrix elements are convoluted with the NNPDF3.0 and CT14 PDF sets.

by the $\tilde{g} \tilde{\chi}_{1}^{+}, \tilde{\chi}_{1}^{+} \tilde{\chi}_{1}^{0}$, and $\tilde{\chi}_{1}^{+} \tilde{\chi}_{2}^{0}$ processes for $\tilde{M} \sim 2.5 \mathrm{TeV}$. The dominant contribution to these three processes originates from a $u \bar{d}$ initial state. However, the NNPDF densities used here are mostly unknown at large $x(x>0.1)$ and are therefore associated with a large uncertainty. Furthermore, the NNPDF methodology (which relies on neural networks to perform the PDF fit) yields the odd shape of the cross sections and $K$-factors in this regime. Correspondingly, the PDF uncertainties related to these processes grow out of control for $\tilde{M}>1.5 \mathrm{TeV}$, and the shape of the central $K$-factor, in particular close to $\tilde{M} \sim 2.5 \mathrm{TeV}$, stems from the Born and real-emission contributions being affected differently by the corresponding partonic luminosities.

In order to better understand these peculiar features of the $\tilde{g} \tilde{\chi}_{1}^{+}, \tilde{\chi}_{1}^{+} \tilde{\chi}_{1}^{0}$ and $\tilde{\chi}_{1}^{+} \tilde{\chi}_{2}^{0}$ cross sections, we show in figure 8 the predictions obtained when the matrix elements are convoluted either with the NNPDF 3.0 NLO PDF set, or with the CT14nlo Hessian PDF set [213]. While the cross sections evaluated with CT14 PDFs show a more reasonable behaviour at large $\tilde{M}$, this comes at the cost of introducing a theoretically-dominated bias on the predictions, as such a PDF set relies entirely, in the large- $x$ region where there is no data point to constrain the fit, on the extrapolation of its parametrisation at the initial scale.

The results obtained by increasing the centre-of-mass energy to $\sqrt{S}=27 \mathrm{TeV}$ and $100 \mathrm{TeV}$ are presented in figures 9 and 10, respectively. As far as the relative comparisons 

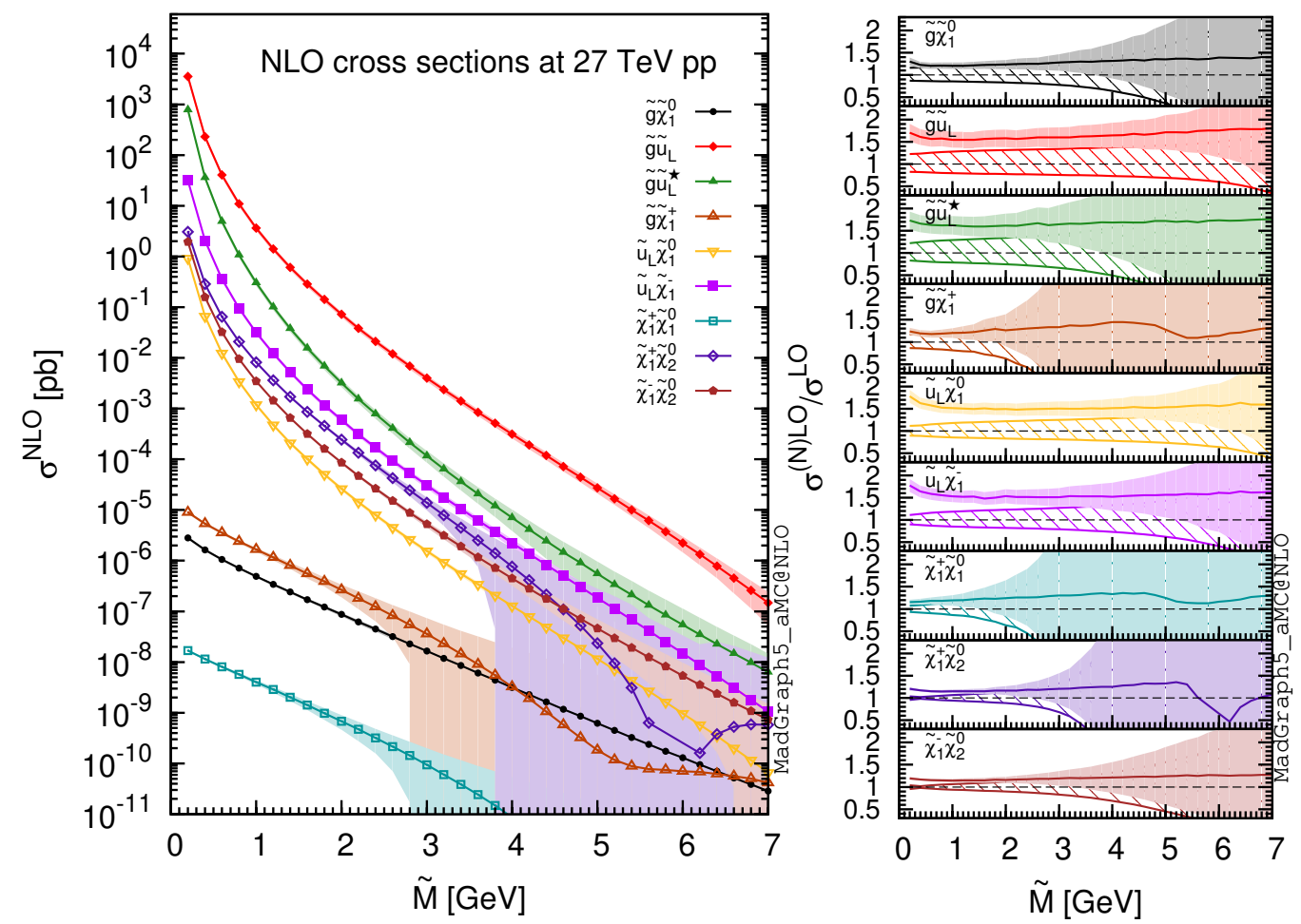

Figure 9. Same as figure 7, but for proton-proton collisions at $\sqrt{S}=27 \mathrm{TeV}$.

of these predictions with those relevant to $\sqrt{S}=13 \mathrm{TeV}$ is concerned, the same observations made at the end of section 4.2 for the case of same-species pair production apply here.

We conclude this section by pointing out that tables reporting the numerical values that correspond to the cross sections shown in figures 4-10 are provided as ancillary files on the electronic archive.

\section{Perturbative computations in the presence of resonances}

In this section, we discuss in general the problems posed to perturbative computations by the presence of narrow resonances, and outline the strategies (called Simplified Treatments of Resonances or STR for short) which one may employ to overcome such problems. STR include all of the Diagram Subtraction (DS) and Diagram Removal (DR) procedures defined so far in the literature, and must be seen as a systematic generalisation of the so-called onshell subtractions. We also document here the implementation of the STR in MG5_AMC. Illustrative examples of their applications are given in section 6 .

\subsection{General features}

In any theory with a sufficiently rich particle spectrum, there is the possibility that the cross section for the production of a given asymptotic state $\delta$ is ill-defined in perturbation theory beyond the LO. Here, $\delta$ is such that its four momentum can, at least in principle, be reconstructed through measurements performed with a realistic detector, either directly 

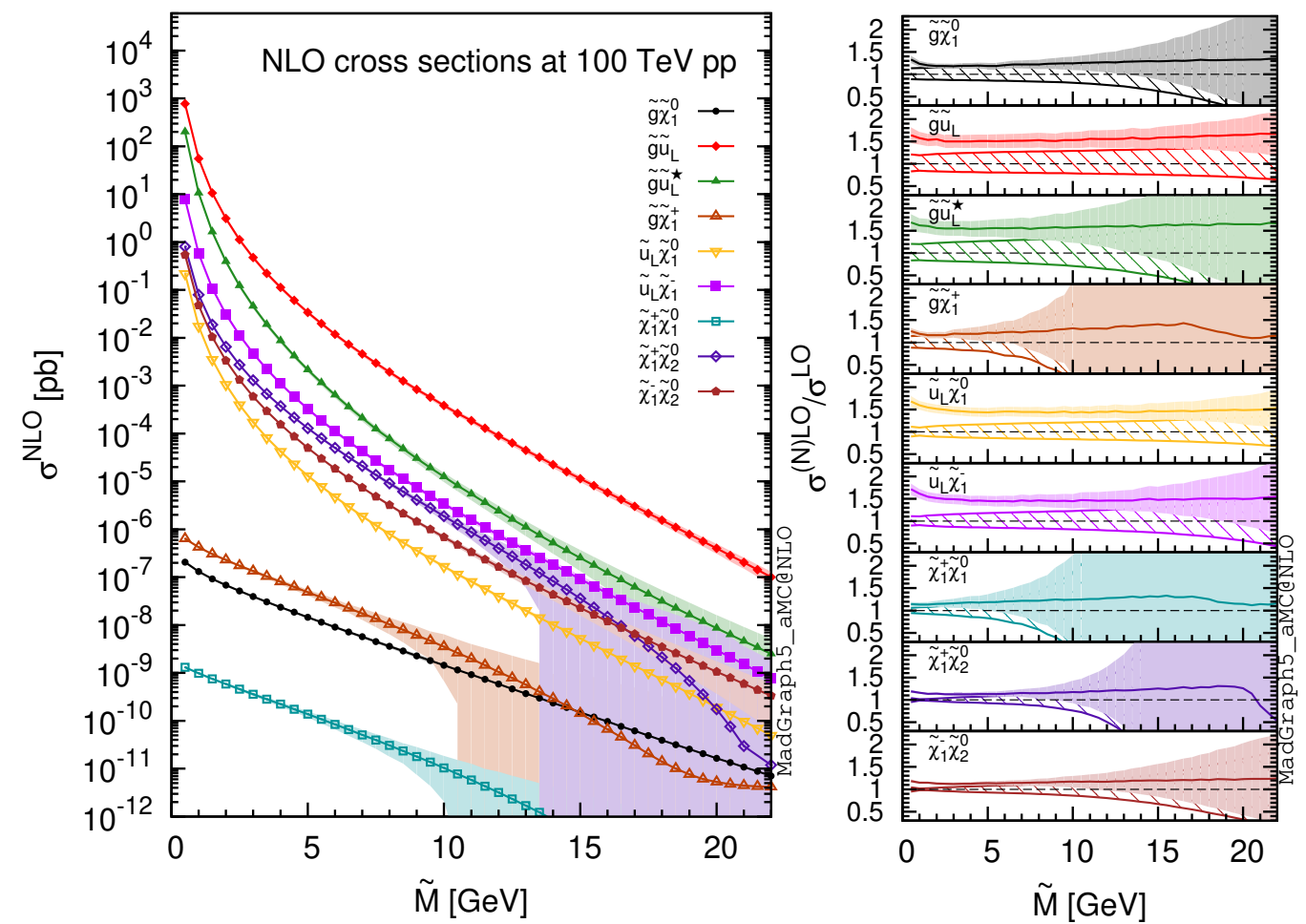

Figure 10. Same as figure 7, but for proton-proton collisions at $\sqrt{S}=100 \mathrm{TeV}$.

or indirectly through its decay products. ${ }^{2}$ This situation can occur in the following case. Let

$$
a+b \longrightarrow \delta+X
$$

be an LO contribution to the production of $\delta$; $a$ and $b$ denote the incoming partons that initiate the hard scattering, and $X$ a set of final-state particles. The cross section for the process of eq. (5.1) may be inclusive or exclusive in $X$. At the NLO, real-emission corrections will receive contributions from processes that one can write as follows:

$$
a+b \longrightarrow \delta+\gamma+X,
$$

where the nature of $\gamma$ depends on the underlying theory whose perturbative expansion is considered. For example, in QCD $\gamma$ can be a massless quark or a gluon, while in QED it can be a photon. Let us now suppose that a particle $\beta$ (which must not resonantly contributes to the process of eq. (5.1)) exists, such that the two-body decay channel

$$
\beta \longrightarrow \delta+\gamma
$$

is kinematically allowed and that

$$
a+b \longrightarrow \beta+X,
$$

\footnotetext{
${ }^{2}$ In other words, $\delta$ is not a light quark or a gluon.
} 
is a well-defined hard process, that we call the underlying resonant process. Equations (5.3) and (5.4) imply that, among the Feynman diagrams contributing to the process of eq. (5.2), there will be $\beta$-resonant ones, namely those that feature a propagator associated with $\beta$. In turn, this allows one to write the contributions of such diagrams to the differential cross section associated with eq. (5.2) as follows:

$$
d \sigma_{a b \rightarrow \delta \gamma X} \stackrel{m_{\delta \gamma} \rightarrow m_{\beta}}{\sim} d \sigma_{a b \rightarrow \beta X} \frac{1}{\left(m_{\delta \gamma}^{2}-m_{\beta}^{2}\right)^{2}} d \Gamma_{\beta \rightarrow \delta \gamma}
$$

where the first term on the r.h.s. is the cross section for the production of an on-shell $\beta$. In eq. (5.5), we have denoted by $m_{\delta \gamma}$ and $m_{\beta}$ the invariant masses of the $\delta \gamma$ pair and of the particle $\beta$, respectively. Even if one Dyson-resums the propagator in eq. (5.5), thus introducing a regularising $\Gamma_{\beta}$ factor that prevents the propagator from diverging at $m_{\delta \gamma}=m_{\beta}$, it may still happen that

$$
\int d \sigma_{a b \rightarrow \beta X} \gg \int d \sigma_{a b \rightarrow \delta X} .
$$

In this case, the NLO contribution due to eq. (5.2) will be numerically (much) larger than its LO counterpart of eq. (5.1), thus 'spoiling' the perturbative expansion of the cross section for $\delta$ production.

Situations of this kind are annoying because of potentially relevant for phenomenology, especially in SUSY theories where they are ubiquitous. Examples stemming from QCD corrections include

$$
(\delta, \gamma, \beta, X)=\left(W^{-}, \bar{b}, \bar{t}, t\right)
$$

in the $\mathrm{SM}\left(t W^{-}\right.$associated production, whose underlying resonant process is $t \bar{t}$ production) and

$$
\begin{aligned}
& (\delta, \gamma, \beta, X)=(\tilde{q}, q, \tilde{g}, \tilde{q}), \\
& (\delta, \gamma, \beta, X)=(\tilde{\chi}, q, \tilde{q}, \tilde{q})
\end{aligned}
$$

in SUSY (squark-pair and squark-neutralino production, whose underlying resonant processes are squark-gluino and squark-pair production, respectively ${ }^{3}$ ). A further case is that of a simplified dark matter model, achieved e.g. by extending the SM with a dark matter particle $\chi$ and a mediator $Y$, so that

$$
(\delta, \gamma, \beta, X)=(\chi, q, Y, \chi)
$$

is the analogue of the processes of eq. (5.7)-(5.9).

By adopting a commonly-used expression, which is strictly speaking incorrect but conveys the basic physics idea, one says that $\delta X$ production interferes with $\beta X$ production beyond the LO. The numerical dominance of the latter over the former implies that the corresponding cross section is, to a good approximation, a meaningful physical quantity

\footnotetext{
${ }^{3}$ Squark-neutralino production features a neutralino-gluino channel which also plays the role of underlying resonant process.
} 
(for example, we are used to talking about measurements of the $t \bar{t}$ cross section, which we compare with their perturbatively-computed counterparts). Conversely, the answer to the question of whether it is possible (and, if so, whether it is sensible and/or convenient) to deal with a perturbative, non- $\beta$-resonant, $\delta X$ cross section depends on the context in which one is working. One can introduce two conceptually different classes of applications:

1. Definition of the non- $\beta$-resonant $\delta X$ cross section as a measurable quantity, for a direct comparison with experimental results.

2. Use of non- $\beta$-resonant $\delta X$ production in conjunction with $\beta X$ production, as perturbative tools that render technically easier the computation of the $\delta X$ cross section that includes both resonant and non-resonant contributions.

As a rule of thumb, applications of class 1 are mostly of interest to SM physics, while those of class 2 are relevant to both the SM and to SUSY (and, in general, to theories which are not confirmed experimentally, and whose signals need to be searched for). By using again the SM example of eq. (5.7), its class 1 applications entail the definition of the $t W^{-}$cross section (see e.g. refs. $[214,215]$ for recent ATLAS and CMS measurements of this quantity at the LHC), for which the underlying $t \bar{t}$ resonant process is considered as a background. On the other hand, in a typical class 2 application one would exploit the possibility of computing both the $t W^{-}$and $t \bar{t}$ cross sections at the NLO (i.e. up to $\mathcal{O}\left(\alpha \alpha_{S}^{2}\right)$ and $\mathcal{O}\left(\alpha_{S}^{3}\right)$, respectively) for a phenomenologically accurate description of $W^{+} W^{-} b(\bar{b})$ production which is much less demanding, from a computational viewpoint, than the calculation of the $W^{+} W^{-} b \bar{b}$ cross section at the NLO.

The key point of the previous example is that the $t W^{-}$cross sections that enter the two applications are not necessarily defined in the same way. In general, let

$$
\mathcal{A}_{a b \rightarrow \delta \gamma X}=\mathcal{A}_{a b \rightarrow \delta \gamma X}^{(\not)}+\mathcal{A}_{a b \rightarrow \delta \gamma X}^{(\beta)}
$$

be the amplitude associated with the process of eq. (5.2). The two quantities on the r.h.s. of eq. (5.11) denote the non- $\beta$-resonant and the $\beta$-resonant contributions, respectively. The matrix element will thus be proportional to:

$$
\left|\mathcal{A}_{a b \rightarrow \delta \gamma X}\right|^{2}=\left|\mathcal{A}_{a b \rightarrow \delta \gamma X}^{(\not)}\right|^{2}+2 \Re\left(\mathcal{A}_{a b \rightarrow \delta \gamma X}^{(\not \beta)} \mathcal{A}_{a b \rightarrow \delta \gamma X}^{(\beta)^{\dagger}}\right)+\left|\mathcal{A}_{a b \rightarrow \delta \gamma X}^{(\beta)}\right|^{2} .
$$

For both class 1 and class 2 applications, the contribution of the last term on the r.h.s. of eq. (5.12) must be minimised. In the context of NLO+PS simulations, this problem has been solved in ref. [216] by introducing two different types of procedures. In diagram removal (DR), one simply drops this contribution, whereas in diagram subtraction (DS) the non- $\beta$-resonant $\delta X$ cross section will feature the linear combination:

$$
\left|\mathcal{A}_{a b \rightarrow \delta \gamma X}^{(\beta)}\right|^{2} d \phi-f\left(m_{\delta \gamma}^{2}\right) \mathbb{P}\left(\left|\mathcal{A}_{a b \rightarrow \delta \gamma X}^{(\beta)}\right|^{2} d \phi\right) .
$$

The pre-factor in the second term of eq. (5.13) is arbitrary to a large extent, but must obey the condition

$$
\lim _{m_{\delta \gamma} \rightarrow m_{\beta}} f\left(m_{\delta \gamma}^{2}\right)=1 .
$$


The symbol $\mathbb{P}$ denotes a kinematic projection that maps a generic $\delta \gamma X$ configuration onto one that has $m_{\delta \gamma}=m_{\beta}$. Crucially, this map is fully local in the phase space, so that the difference in eq. (5.13) vanishes identically when $m_{\delta \gamma} \rightarrow m_{\beta}$ (also thanks to eq. (5.14)); such a locality condition is essential for the use of DS in event generators. Owing to the definition of the $\beta$-resonant amplitude, one has

$$
\mathbb{P}\left(\left|\mathcal{A}_{a b \rightarrow \delta \gamma X}^{(\beta)}\right|^{2}\right) \propto\left|\mathcal{A}_{a b \rightarrow \beta X}\right|^{2}
$$

by neglecting production spin correlations. Thus, eq. (5.15) renders it intuitively clear that the difference in eq. (5.13) is constructed so as to avoid the double counting of the LO $\beta X$ cross section in class 2 approaches. In practice, spin correlations cannot be neglected, and therefore $\mathcal{A}_{a b \rightarrow \beta X}$ is never used as such in DS procedures; ${ }^{4}$ this is just as well, since it helps to guarantee the local cancellation between the two terms in eq. (5.13). We finally stress that there is ample freedom in the choices of the function $f$ and projector $\mathbb{P}$ that enter the definition of a DS cross section through eq. (5.13). We shall exploit this fact in the following, by considering several different implementations.

As far as the second term on the r.h.s. of eq. (5.12) is concerned, DR procedures do not include it in the definition of the non- $\beta$-resonant $\delta X$ cross section, while DS procedures do. Thus, for class 1 applications it is essential that both DR and DS results be obtained, and that their difference be less than the theoretical systematics. If that is not the case, non- $\beta$-resonant $\delta X$ observables are simply not physically meaningful, and both DR and DS predictions must be discarded. Conversely, for class 2 applications, in which the emphasis is on obtaining the best approximation for the full $\delta X$ cross section, one is interested in using DS approaches. DR results might also be kept, provided they are statistically compatible with the DS ones. We also point out that in the NLO+PS simulations of ref. [185] a third scenario has been considered (dubbed DR2 there, and originally introduced in ref. [116] within a fixed-order calculation), in which one keeps the second term on the r.h.s. of eq. (5.12), but does not perform the subtraction of eq. (5.13). As far as its usage in applications of class 1 and 2 is concerned, this approach is quite analogous to a DS one. However, given that no subtraction is carried out, we call it DR+I (for diagram removal plus interference).

In summary, the non- $\beta$-resonant $\delta \gamma X$ cross section can be defined as follows:

$$
\begin{aligned}
d \sigma_{a b \rightarrow \delta \gamma X}^{(\mathrm{DR})} \propto & \left|\mathcal{A}_{a b \rightarrow \delta \gamma X}^{(\not \beta)}\right|^{2} d \phi \\
d \sigma_{a b \rightarrow \delta \gamma X}^{(\mathrm{DR}+\mathrm{I})} \propto & \left\{\left|\mathcal{A}_{a b \rightarrow \delta \gamma X}^{(\not \beta)}\right|^{2}+2 \Re\left(\mathcal{A}_{a b \rightarrow \delta \gamma X}^{(\not \beta)} \mathcal{A}_{a b \rightarrow \delta \gamma X}^{(\beta)^{\dagger}}\right)\right\} d \phi, \\
d \sigma_{a b \rightarrow \delta \gamma X}^{(\mathrm{DS})} \propto & \left\{\left|\mathcal{A}_{a b \rightarrow \delta \gamma X}^{(\not \beta)}\right|^{2}+2 \Re\left(\mathcal{A}_{a b \rightarrow \delta \gamma X}^{(\not \beta)} \mathcal{A}_{a b \rightarrow \delta \gamma X}^{(\beta)^{\dagger}}\right)+\left|\mathcal{A}_{a b \rightarrow \delta \gamma X}^{(\beta)}\right|^{2}\right\} d \phi \\
& -f\left(m_{\delta \gamma}^{2}\right) \mathbb{P}\left(\left|\mathcal{A}_{a b \rightarrow \delta \gamma X}^{(\beta)}\right|^{2} d \phi\right),
\end{aligned}
$$

\footnotetext{
${ }^{4}$ It is indeed $\mathcal{A}_{a b \rightarrow \delta \gamma X}^{(\beta)}$ (suitably projected) that is employed, in the method of ref. [217], to include production spin correlations at the tree level in the Monte Carlo simulations of $\beta X$ production.
} 
in the DR, DR+I, and DS approaches, respectively. Collectively, the procedures implied by eqs. (5.16)-(5.18) will be called STR (that stands for Simplified Treatments of Resonances).

STR strategies have been pursued for a long while in the context of fixed-order calculations and for inclusive observables in both the SM and BSM theories (in particular in SUSY, where they are typically called OS subtractions) - see e.g. refs. [93, 116, 218-223]. None of these earlier procedures is apt to be applied to exclusive event generation, and thus we believe one should refrain from using the DR or DS tags in association with them. As far as DR and DS procedures are concerned, either identical to or featuring variants of those originally proposed in ref. [216], results can be found in refs. [142, 143, 148, 149, 185, 224-226].

We now turn to giving some details about the implementation of eqs. (5.16)-(5.18) in MG5_AMC. In keeping with the general strategy that underpins the code, everything is fully automated and process- as well as model-independent. ${ }^{5}$ We remind the reader that MG5_AMC is self-consistent, and thus that, in particular, it generates internally the Feynman diagrams and writes the corresponding amplitudes. This implies that the code stores the information on the topological structure of each diagram, and therefore knows where to find the resonances (which is essential in order to construct $\mathcal{A}_{a b \rightarrow \delta \gamma X}^{(\not)}$ and $\mathcal{A}_{a b \rightarrow \delta \gamma X}^{(\beta)}$ ). Furthermore, it can control the kinematical inputs and parameter settings in a diagram-bydiagram manner if needed. The immediate consequence of the previous observation is that the construction of the DR and DR+I cross sections of eqs. (5.16) and (5.17), respectively, is achieved in a straightforward (and unique) manner.

The case of the DS cross section is more involved, owing to the freedom in the definitions of the function $f$ and of the projector $\mathbb{P}$, although after having chosen $f$ and $\mathbb{P}$, eq. (5.18) uniquely determines the corresponding DS procedure. Unfortunately, it is impossible to parametrise the arbitrariness in the choices of $f$ and $\mathbb{P}$, and thus one must limit oneself to considering a finite number of physically-motivated options. We describe those implemented in MG5_AMC below, and point out that previous results in the DS approach $[142,143,148,149,216,224-226]$ have been obtained with a given $(f, \mathbb{P})$ pair (with the exception of ref. [185], where two different forms of $f$ have been compared).

\subsection{Diagram-subtraction procedures}

We start by pointing out that the discussion that follows is relevant to the last term on the r.h.s. of eq. (5.18), henceforth called the DS subtraction term. The other three terms in the definition of the DS cross section correspond to a straightforward tree-level calculation, and are thus not of concern here. We denote the kinematic of the process of eq. (5.2) as follows:

$$
k_{a}+k_{b}=k_{\delta}+k_{\gamma}+\sum_{i=1}^{n-1} k_{i},
$$

\footnotetext{
${ }^{5}$ Some limitations exist, as STR procedures cannot for instance be used within MADSPIN [227] or the reweighting module of MG5_AMC [228].
} 
where we have assumed that the set $X$ is composed of $n-1$ particles with momenta $k_{i}$. It is convenient to introduce the following auxiliary momenta:

$$
\begin{aligned}
q & =k_{\beta}+k_{\mathrm{rec}}=k_{a}+k_{b}, \\
k_{\beta} & =k_{\delta}+k_{\gamma}, \\
k_{\mathrm{rec}} & =\sum_{i=1}^{n-1} k_{i} .
\end{aligned}
$$

Although the resonance $\beta$ does not appear in the final state, the definition of its momentum in eq. (5.21) is physically meaningful, since we are solely dealing with $\beta$-resonant diagrams. In the centre-of-mass frame of the incoming hadrons:

$$
k_{a}=x_{a} \frac{\sqrt{S}}{2}(1,0,0,1) \quad \text { and } \quad k_{b}=x_{b} \frac{\sqrt{S}}{2}(1,0,0,-1),
$$

with $S$ being the squared hadronic centre-of-mass energy. Its parton-level counterpart reads thus:

$$
s \equiv q^{2}=\left(k_{a}+k_{b}\right)^{2}=x_{a} x_{b} S .
$$

The action of the projection $\mathbb{P}$ on the partonic kinematic configuration is denoted as the following transformation:

$$
k_{\delta} \longrightarrow \bar{k}_{\delta}, \quad k_{\gamma} \longrightarrow \bar{k}_{\gamma}, \quad k_{i} \longrightarrow \bar{k}_{i} \quad(1 \leq i \leq n-1) .
$$

We also introduce, for consistency with eq. (5.25), the momentum of the projected resonance

$$
k_{\beta} \longrightarrow \bar{k}_{\beta}, \quad \bar{k}_{\beta}=\bar{k}_{\delta}+\bar{k}_{\gamma} .
$$

The DS strategies that we consider generally require the partonic incoming momenta to be changed. This can formally be seen as also stemming from the action of $\mathbb{P}$, and thus be represented as follows:

$$
k_{a} \longrightarrow \bar{k}_{a}, \quad k_{b} \longrightarrow \bar{k}_{b}
$$

By taking eqs. (5.23) and (5.24) into account, eq. (5.27) can be equivalently written as the transformation

$$
x_{a} \longrightarrow \bar{x}_{a}, \quad x_{b} \longrightarrow \bar{x}_{b}, \quad s \longrightarrow \bar{s} \equiv \bar{x}_{a} \bar{x}_{b} S .
$$

While the specific form of eq. (5.28) will depend on $\mathbb{P}$, in all of our implementations we shall always choose $\bar{x}_{a}$ and $\bar{x}_{b}$ so that

$$
\frac{\bar{x}_{a}}{\bar{x}_{b}}=\frac{x_{a}}{x_{b}} .
$$

This implies that the original and projected partonic centre-of-mass frames will travel at the same speed w.r.t. the hadronic centre-of-mass frame. Equation (5.28) has two further implications. Firstly, the flux factor of the DS subtraction term is equal to $1 /(2 \bar{s})$. Secondly, its parton-luminosity factor is given by

$$
f_{a}^{\left(H_{1}\right)}\left(\bar{x}_{a}\right) f_{b}^{\left(H_{2}\right)}\left(\bar{x}_{b}\right)
$$

with $H_{1}$ and $H_{2}$ being the incoming hadrons. 
As far as the function $f$ is concerned, we shall limit ourselves to considering the following form:

$$
f\left(m^{2}\right)=\frac{\mathrm{BW}_{\beta}\left(m^{2}, x\right)}{\mathrm{BW}_{\beta}\left(m_{\beta}^{2}, x\right)},
$$

for a given choice of $x$, and where

$$
\mathrm{BW}_{\beta}\left(m^{2}, x\right)=\frac{\kappa}{\left(m^{2}-m_{\beta}^{2}\right)^{2}+\left(x \Gamma_{\beta}\right)^{2}}
$$

is a generalised Breit-Wigner function (the standard one being obtained by setting $x=m_{\beta}$ ) in which $\kappa$ is a normalisation factor that does not play any role. The rationale beyond eq. (5.31) is that its denominator will cancel, to some extent, an analogous term implicit in the projected matrix element of the DS subtraction term, that is thus replaced by the numerator of eq. (5.31) which supposedly models some of the off-shell- $\beta$ effects. The precise extent of such a cancellation depends on the interplay of several factors (such as the choice of $x$, of the operator $\mathbb{P}$, or of the PDFs), and cannot therefore be predicted a priori. This is one of the reasons why in eq. (5.31) $x$ is treated as a free parameter.

In view of their use in the definition of $\mathbb{P}$, we also introduce the following quantities. For any four-momentum $p$, we denote the boost to its rest frame by:

$$
\mathbb{B}_{R}(p) p=(m, \overrightarrow{0}) .
$$

This understands that $p^{2}=m^{2}>0$; we implicitly assume that the boost is performed along $\vec{p}$, and that it is such that:

$$
\exp \left(y_{\mathbb{B}_{R}}\right)=\sqrt{\frac{E+|\vec{p}|}{E-|\vec{p}|}}, \quad p=(E, \vec{p}) \equiv\left(\sqrt{m^{2}+|\vec{p}|^{2}}, \vec{p}\right) .
$$

If in the rest frame of $p$ we impose a $1 \rightarrow 2$ four-momentum conservation,

$$
(m, \overrightarrow{0})=p_{1}+p_{2},
$$

then by denoting $p_{i}^{2}=m_{i}^{2}$ (with $m>m_{1}+m_{2}$ ), we have

$$
\begin{aligned}
& p_{1}=\left(\varepsilon\left(m, m_{1}, m_{2}\right), \pi\left(m, m_{1}, m_{2}\right) \vec{e}\right), \\
& p_{2}=\left(\varepsilon\left(m, m_{2}, m_{1}\right),-\pi\left(m, m_{1}, m_{2}\right) \vec{e}\right),
\end{aligned}
$$

where $|\vec{e}|=1$ and:

$$
\begin{aligned}
\pi\left(m, m_{1}, m_{2}\right) & =\frac{m}{2} \lambda\left(m, m_{1}, m_{2}\right), \\
\varepsilon\left(m, m_{1}, m_{2}\right) & =\sqrt{m_{1}^{2}+\pi\left(m, m_{1}, m_{2}\right)^{2}} \\
& =\frac{m}{2}\left(1+\frac{m_{1}^{2}-m_{2}^{2}}{m^{2}}\right),
\end{aligned}
$$


with:

$$
\lambda(a, b, c)=\sqrt{1-\frac{(b+c)^{2}}{a^{2}}} \sqrt{1-\frac{(b-c)^{2}}{a^{2}}} .
$$

We can now present specific details of the definition of the DS subtraction term in the DS procedures we pursue. The reader must keep in mind that the kinematic configuration of eq. (5.19) and its associated quantities eqs. (5.20)-(5.22) are thought to be given. Without loss of generality, we work in the incoming-parton centre-of-mass frame, $\vec{q}=\overrightarrow{0}$, and the options described below are associated in MG5_AMC with the function $f$ given in eq. (5.31) and with either of the settings:

$$
x=m_{\beta} \quad \text { or } \quad x=m_{\delta \gamma} .
$$

Other choices of $x$ would be straightforward to implement.

Option A. This option follows the strategy first introduced in ref. [216]. We define:

$$
\begin{aligned}
\vec{k}_{\beta} & =\vec{k}_{\beta}, \\
\bar{k}_{\beta}^{0} & =\sqrt{m_{\beta}^{2}+\vec{k}_{\beta}^{2}} .
\end{aligned}
$$

The momenta not associated with the resonance $\beta$ are left invariant:

$$
\bar{k}_{i}=k_{i} \quad 1 \leq i \leq n-1
$$

In view of eqs. (5.43) and (5.44), we then define:

$$
\sqrt{\bar{s}}=\bar{q}^{0}=\bar{k}_{\beta}^{0}+\sum_{i=1}^{n-1} \bar{k}_{i}^{0} .
$$

As far as the momenta of $\delta$ and $\gamma$ are concerned, we proceed as follows. First, we boost them in the rest frame of $k_{\beta}$ :

$$
\begin{aligned}
& \mathbb{B}_{R}\left(k_{\beta}\right) k_{\delta}=\left(\varepsilon\left(\sqrt{k_{\beta}^{2}}, m_{\delta}, m_{\gamma}\right), \pi\left(\sqrt{k_{\beta}^{2}}, m_{\delta}, m_{\gamma}\right) \vec{e}_{\delta}\right), \\
& \mathbb{B}_{R}\left(k_{\beta}\right) k_{\gamma}=\left(\varepsilon\left(\sqrt{k_{\beta}^{2}}, m_{\gamma}, m_{\delta}\right),-\pi\left(\sqrt{k_{\beta}^{2}}, m_{\delta}, m_{\gamma}\right) \vec{e}_{\delta}\right),
\end{aligned}
$$

owing to eqs. (5.35)-(5.36). Then, by keeping the information on $\vec{e}_{\delta}$ but discarding all the rest, we define

$$
\begin{aligned}
& \bar{k}_{\delta}=\mathbb{B}_{R}^{-1}\left(\bar{k}_{\beta}\right)\left(\varepsilon\left(m_{\beta}, m_{\delta}, m_{\gamma}\right), \pi\left(m_{\beta}, m_{\delta}, m_{\gamma}\right) \vec{e}_{\delta}\right) \\
& \bar{k}_{\gamma}=\mathbb{B}_{R}^{-1}\left(\bar{k}_{\beta}\right)\left(\varepsilon\left(m_{\beta}, m_{\delta}, m_{\gamma}\right),-\pi\left(m_{\beta}, m_{\delta}, m_{\gamma}\right) \vec{e}_{\delta}\right)
\end{aligned}
$$

which guarantee consistency with eqs. (5.42) and (5.43), and thus ultimately enforce fourmomentum conservation. In the case where $k_{\beta}^{2}=m_{\beta}^{2}$, all of the operations above are equivalent to the identity. 
Option B. This option generalises (to an arbitrary number of final-state particles) the strategy of ref. [142]. We define the mass of the recoil system $X$ :

$$
m_{\mathrm{rec}}^{2}=k_{\mathrm{rec}}^{2}
$$

and we keep it invariant. If the condition

$$
\sqrt{s} \geq m_{\beta}+m_{\mathrm{rec}}
$$

is fulfilled, we then set

$$
\bar{s}=s .
$$

Otherwise, we define: ${ }^{6}$

$$
\sqrt{\bar{s}}=\frac{m_{\beta}+m_{\mathrm{rec}}}{\sqrt{k_{\beta}^{2}}+m_{\mathrm{rec}}} \sqrt{s} .
$$

Next, we define the energies of the projected resonance and recoil system as follows:

$$
\begin{aligned}
\bar{k}_{\beta}^{0} & =\varepsilon\left(\sqrt{\bar{s}}, m_{\beta}, m_{\mathrm{rec}}\right), \\
\bar{k}_{\mathrm{rec}}^{0} & =\varepsilon\left(\sqrt{\bar{s}}, m_{\mathrm{rec}}, m_{\beta}\right) .
\end{aligned}
$$

The corresponding three-momenta are defined by preserving the direction of the original three-momenta, rescaling their lengths so as to impose the mass shell conditions

$$
\begin{gathered}
\overrightarrow{\vec{k}}_{\beta}=\sqrt{\left(\bar{k}_{\beta}^{0}\right)^{2}-m_{\beta}^{2}} \frac{\vec{k}_{\beta}}{\left|\vec{k}_{\beta}\right|}=\pi\left(\sqrt{\bar{s}}, m_{\beta}, m_{\mathrm{rec}}\right) \frac{\vec{k}_{\beta}}{\left|\vec{k}_{\beta}\right|}, \\
\overrightarrow{\vec{k}}_{\mathrm{rec}}=\sqrt{\left(\bar{k}_{\mathrm{rec}}^{0}\right)^{2}-m_{\mathrm{rec}}^{2}} \frac{\vec{k}_{\mathrm{rec}}}{\left|\vec{k}_{\mathrm{rec}}\right|}=\pi\left(\sqrt{\bar{s}}, m_{\beta}, m_{\mathrm{rec}}\right) \frac{\vec{k}_{\mathrm{rec}}}{\left|\vec{k}_{\mathrm{rec}}\right|} .
\end{gathered}
$$

These guarantee that $\vec{k}_{\beta}=-\overrightarrow{\vec{k}}_{\text {rec}}$, since $\vec{k}_{\beta}=-\vec{k}_{\text {rec }}$. We also define

$$
\bar{k}_{i}=\mathbb{B}_{R}^{-1}\left(\bar{k}_{\mathrm{rec}}\right) \mathbb{B}_{R}\left(k_{\mathrm{rec}}\right) k_{i} \quad \text { for } \quad 1 \leq i \leq n-1 .
$$

Finally, $k_{\delta}$ and $k_{\gamma}$ are projected using the same procedure as in eqs. (5.46)-(5.49).

Option C. This option, which is currently not implemented in MG5_AMC, follows closely what is done for the phase-space generation as is carried out in the module MADFKS [156], in the case relevant to a massive FKS sister. This, in turn, generalises the massless-parton treatment of ref. [229]. The projected partonic centre-of-mass energy is defined as follows:

$$
\sqrt{\bar{s}}=\frac{m_{\beta}+\sum_{i=1}^{n-1} m_{i}}{\min \left(\sqrt{k_{\beta}^{2}}, \varsigma m_{\beta}\right)+\sum_{i=1}^{n-1} m_{i}} \sqrt{s},
$$

\footnotetext{
${ }^{6}$ Alternatively, one can leave invariant the partonic centre-of-mass energy, and assign to $k_{\beta}^{2}$ the largest value compatible with that energy. This has the disadvantage of defining a DS subtraction term which does not correspond to an on-shell $\beta$-resonant cross section.
} 
with $\varsigma \geq 1$ being a free parameter, ${ }^{7}$ typically of $\mathcal{O}(1)$. One then regenerates the final-state kinematic configuration, using the same random numbers as for the original one, and $\bar{s}$ instead of $s$ (the configuration thus obtained has only a temporary role, and is denoted below in the same way as the original one). Next, a boost $\mathbb{B}$ is defined along the resonance three-momentum $\vec{k}_{\beta}$, so that:

$$
\left(\bar{q}-\mathbb{B} k_{\text {rec }}\right)^{2}=m_{\beta}^{2} .
$$

The projected momenta are then given by:

$$
\bar{k}_{\beta}=\bar{q}-\mathbb{B} k_{\text {rec }} \quad \text { and } \quad \bar{k}_{i}=\mathbb{B} k_{i} .
$$

Once again, the $k_{\delta}$ and $k_{\gamma}$ momenta are projected using the same procedure as in eqs. (5.46)-(5.49).

\subsection{Using DR and DS in MG5_AMC}

All of the STR procedures described above can be employed within MG5_AMC by downloading the MADSTR plugin, ${ }^{8}$ and by copying the directory MadSTR thus obtained inside the PLUGIN directory, which is part of any (recent) MG5_AMC release. The current version of MADSTR is compatible with MG5_AMC version 2.6 and higher; compatibility with versions 3 and higher, that are capable of carrying out mixed-coupling perturbative computations [70], will be added in the future. The plugin can be activated by using the following command to start MG5_AMC:

$$
\text { mg5_aMC --mode=MadSTR }
$$

One can then generate NLO processes and write them to disk as usual (with the generate and output commands - see ref. [56] for more details). The plugin will take care of identifying any potentially resonant contributions, of generating the associated underlying resonant processes, and of taking care of the extra bookkeeping in addition to that normally performed by MG5_AMC in non-resonant cases. Depending on the mass spectrum, the contributions for which the STR is needed are identified at run-time. Three parameters found in run_card.dat, namely istr, str_include_flux, and str_include_pdf, can be used to choose the desired STR procedure and its associated options. More specifically, the type of STR is controlled by istr, which must assigned an integer value according to the options given in table 2. The other two parameters, str_include_flux and str_include_pdf, are active only if the STR is of DS type, and control the settings of the flux and the parton luminosity factors, respectively, in the DS subtraction terms. In particular, DS procedures imply changes to the partonic centre-of-mass energy (see eqs. (5.45) and (5.53)). In turn, this seemingly implies that the flux and the luminosity factors should be changed accordingly, when the partonic centre-of-mass energy is changed. However, this is not mandatory, since it is actually part of the definition of the projection inherent to DS procedures and,

\footnotetext{
${ }^{7}$ It is also possible to use here the strategy outlined in eqs. (5.51)-(5.53), with the formal replacement $m_{\text {rec }} \rightarrow \sum_{i=1}^{n-1} m_{i}$ there. Likewise, eq. (5.59), with $\sum_{i=1}^{n-1} m_{i} \rightarrow m_{\text {rec }}$, can be used in option B instead of eqs. (5.51)-(5.53).

${ }^{8}$ https://code.launchpad.net/ maddevelopers/mg5amcnlo/MadSTRPlugin.
} 


\begin{tabular}{|cl|}
\hline istr & STR procedure \\
\hline 1 & DR \\
2 & DR+I \\
3 & DS, option A, with $x=m_{\beta}$ \\
4 & DS, option A, with $x=m_{\delta \gamma}$ \\
5 & DS, option B, with $x=m_{\beta}$ \\
6 & DS, option B, with $x=m_{\delta \gamma}$ \\
\hline
\end{tabular}

Table 2. Possible values for the istr parameter, with the corresponding STR procedure. In the case of option A (and of option B when the condition in eq. (5.51) is not fulfilled), the parameters str_include_flux and str_include_pdf are also relevant (see text for details).

as such, is liable to be chosen by the user. It is in order to give one the possibility of exploring the consequence of this choice that the parameters str_include_flux and str_include_pdf have been introduced. By setting them equal to True (False), the flux and PDF factors are (not) re-defined. Note that the settings of the two parameters are independent from each other, and that True are the default values. More details in the context of a specific example will be given in section 6 .

Finally, the value of $\Gamma_{\beta}$ (which acts as a regulator when $m_{\delta \gamma} \rightarrow m_{\beta}$ ) can be controlled by changing the width of the corresponding particle $\beta$ in the param_card.dat file, for all $\beta$ 's that are potentially resonant. The code will set the widths of all coloured particles ${ }^{9}$ equal to zero everywhere except in the resonant real-emission diagrams and in the corresponding DS subtraction terms, in which the values provided in the param_card.dat will be employed.

\section{A case study: jets plus missing energy at the NLO+PS accuracy}

We are now in the position to perform phenomenology studies in the MSSM with a generic particle mass spectrum. As an illustrative example, we choose the benchmark point presented in table 3, which is not excluded by current experimental searches at the LHC, and that features non-trivial decay patterns. In contrast with section 2 , the bottom squarks are taken to be non-mixing.

In the scenario of table 3 , the total widths and the relevant decay modes of the gluino and the squarks are as reported in table 4, together with the associated branching ratios, these results being computed at the LO with MadWidth [227]. Thus, the decay widths of the squarks and gluino are sufficiently small relatively to their masses (except for $\tilde{t}_{1}$, which is however never resonant in the processes considered in this section) so that the narrow-width approximation is sensible. Conversely, and according to the parametrisation of long-distance effects e.g. in Pythia8.2 and HeRwig (see refs. [230-232]), the sparticle widths are sufficiently large to avoid hadronisation before decay - in other words, no $R$-hadrons will be present in our simulations.

\footnotetext{
${ }^{9}$ This is because at the moment we restrict ourselves to the case of QCD corrections.
} 


\begin{tabular}{|cl|cl|}
\hline Parameter & value & Parameter & value \\
\hline$m_{t}$ & $172 \mathrm{GeV}$ & $n_{l f}$ & 5 \\
$m_{\tilde{g}}$ & $2000 \mathrm{GeV}$ & $m_{\tilde{u}_{R}}$ & $1200 \mathrm{GeV}$ \\
$m_{\tilde{t}_{1}}$ & $3000 \mathrm{GeV}$ & $m_{\tilde{t}_{2}}$ & $3500 \mathrm{GeV}$ \\
$m_{\tilde{\chi}_{1}^{0}}$ & $50 \mathrm{GeV}$ & $m_{\tilde{q}, \tilde{q} \neq \tilde{t}_{1}, \tilde{t}_{2}, \tilde{u}_{R}}$ & $2500 \mathrm{GeV}$ \\
$m_{\tilde{\chi}_{i}^{0}, i>1}$ & $5500 \mathrm{GeV}$ & $m_{\tilde{\chi}^{ \pm}, \tilde{\ell}^{\mp}}$ & $5500 \mathrm{GeV}$ \\
\hline
\end{tabular}

Table 3. The benchmark scenario used for our phenomenological study.

\begin{tabular}{|c|c|c|c|}
\hline Particle & Width $[\mathrm{GeV}]$ & Decay mode & Branching ratio [\%] \\
\hline \multirow{2}{*}{$\tilde{g}$} & \multirow{2}{*}{16.6} & $\tilde{u}_{R} \bar{u}$ & 50 \\
\hline & & $\tilde{u}_{R}^{\star} u$ & 50 \\
\hline$\tilde{u}_{R}$ & 2.71 & $\tilde{\chi}_{1}^{0} u$ & 100 \\
\hline \multirow{3}{*}{$\tilde{t}_{1}$} & \multirow{3}{*}{534} & $W^{+} \tilde{b}_{L}$ & 91.1 \\
\hline & & $\tilde{g} t$ & 8.8 \\
\hline & & $\tilde{\chi}_{1}^{0} t$ & 0.1 \\
\hline \multirow{3}{*}{$\tilde{t}_{2}$} & \multirow{3}{*}{90.3} & $\tilde{g} t$ & 88.7 \\
\hline & & $\tilde{\chi}_{1}^{0} t$ & 8.8 \\
\hline & & $\tilde{b}_{L} b t$ & 2.5 \\
\hline \multirow{2}{*}{$\tilde{q}_{R}$ with $\tilde{q} \neq \tilde{u}$} & \multirow{2}{*}{18.5} & $\tilde{g} q$ & 92.3 \\
\hline & & $\tilde{\chi}_{1}^{0} q$ & 7.7 \\
\hline \multirow{2}{*}{$\tilde{b}_{L}$} & \multirow{2}{*}{17.4} & $\tilde{g} b$ & 98.0 \\
\hline & & $\tilde{\chi}_{1}^{0} b$ & 2.0 \\
\hline \multirow{3}{*}{$\tilde{q}_{L}$ with $\tilde{q} \neq \tilde{b}$} & \multirow{3}{*}{17.6} & $\tilde{g} q$ & 97.0 \\
\hline & & $\tilde{\chi}_{1}^{0} q$ & 2.0 \\
\hline & & $W \tilde{g} q^{\prime}$ & 1.0 \\
\hline
\end{tabular}

Table 4. Decay widths and branching ratios of gluino and squarks, in the benchmark point of table 3. $q^{\prime}$ denotes the down-type (up-type) quark associated with the same-generation up-type (down-type) quark $q$.

We shall now consider, at the $13 \mathrm{TeV}$ LHC, multijet plus missing transverse-energy final states:

$$
p p \longrightarrow n j+\mathbb{E}_{T},
$$

a signature that is typical of SUSY searches at hadron colliders. We shall compute the contributions to eq. (6.1) due to the following underlying processes:

$$
\begin{array}{ll}
p p \longrightarrow \tilde{g} \tilde{g}+X & \text { denoted by } \tilde{g} \tilde{g}, \\
p p \longrightarrow \tilde{g} \tilde{q}^{(\star)}+X & \text { denoted by } \tilde{g} \tilde{q}, \\
p p \longrightarrow \tilde{q}_{i}^{(\star)} \tilde{q}_{j}^{(\star)}+X & \text { denoted by } \tilde{q} \tilde{q} .
\end{array}
$$




\begin{tabular}{|c|ccc|}
\hline Process & Born signature(s) & Underlying resonant(s) & Decay(s) \\
\hline$\tilde{g} \tilde{g}$ & $4 j+\mathbb{E}_{T}$ & $\tilde{g} \tilde{q}_{h}$ & $\tilde{q}_{h} \rightarrow \tilde{g} q$ \\
\hline$\tilde{g} \tilde{u}_{R}^{(\star)}$ & $3 j+\mathbb{E}_{T}$ & $\tilde{g} \tilde{g}$ & $\tilde{g} \rightarrow \tilde{u}_{R}^{(\star)} u$ \\
& & $\tilde{u}_{R}^{(\star)} \tilde{q}_{h}$ & $\tilde{q}_{h} \rightarrow \tilde{g} q$ \\
$\tilde{g} \tilde{q}_{h}$ & $5 j+\mathbb{E}_{T}$ & $\tilde{q}_{h} \tilde{q}_{h}$ & $\tilde{q}_{h} \rightarrow \tilde{g} q$ \\
\hline$\tilde{u}_{R}^{(\star)} \tilde{u}_{R}^{(\star)}$ & $2 j+\mathbb{E}_{T}$ & $\tilde{g} \tilde{u}_{R}^{(\star)}$ & $\tilde{g} \rightarrow \tilde{u}_{R}^{(\star)} u$ \\
$\tilde{u}_{R}^{(\star)} \tilde{q}_{h}$ & $4 j+\mathbb{E}_{T}$ & $\tilde{g} \tilde{q}_{h}$ & $\tilde{g} \rightarrow \tilde{u}_{R}^{(\star)} u$ \\
$\tilde{q}_{h} \tilde{q}_{h}$ & $6 j+\mathbb{E}_{T}$ & - & - \\
\hline
\end{tabular}

Table 5. Contributions to the signatures of eq. (6.1) stemming from the processes of eqs. (6.2)(6.4). For each of these, we report the Born-level signature (second column), the underlying resonant process (third column), and the decay of the would-be resonant sparticles (fourth column).

The subsequent hadronic decays of the final-state sparticles are carried out by the parton shower programme according to the results of table 4; sample diagrams, corresponding to the signatures of eq. (6.1) being induced by the processes of eqs. (6.2)-(6.4) plus sparticle decays, are depicted in figure 11. In order to ensure that squarks only decay into lightflavoured jets, we restrict our simulations by solely considering the first two generations of squarks. We also distinguish, in the rest of this section, the light (anti-)squark $\tilde{u}_{R}^{(\star)}$ from all of the other heavier (anti-)squarks of the first two generations, that we denote by $\tilde{q}_{h}$.

The situation is summarised in table 5. For each of the processes of eqs. (6.2)-(6.4), which we also distinguish according to whether a light squark is present in the primary (i.e. before decay) process, we report the Born-level signature (second column), the underlying resonant process, defined according to eq. (5.4) (third column), and the relevant decays of the primary sparticles, according to eq. (5.3) (fourth column). The experimental signature of eq. (6.1) is obtained by considering the parton-level process definitions of eqs. (6.2)-(6.4) whose Born contributions do not feature any resonance. ${ }^{10}$ Their corresponding real-emission contributions therefore include at most one SUSY resonance which is then necessarily subject to an STR procedure. In this way, our simulation setup is guaranteed to remain within the scope of STR applicability as described in section 5.1.

All of our simulations are NLO+PS accurate, whereby NLO matrix elements are matched with the PythiA8.2 [233] parton showers according to the MC@NLO formalism [34], automated in MG5_AMC. The resulting hadron-level events are clustered by making use of the anti- $k_{T}$ algorithm [234] with jet radius $R=0.4$, as implemented in FASTJET [235].

For our phenomenological analysis, we implement an event selection similar to the one of the CMS SUSY search of ref. [236]. Firstly, jets are required to have transverse momentum larger than $30 \mathrm{GeV}$, and pseudorapidity $|\eta|<2.4$. Events are kept if they feature at least $N_{\text {jet }} \geq 2$ jets. Secondly, the total hadronic activity $H_{T}$, defined as the

\footnotetext{
${ }^{10}$ This implies that the SUSY particle decays shown in figure 11 must be handled at the level of the parton shower programme.
} 


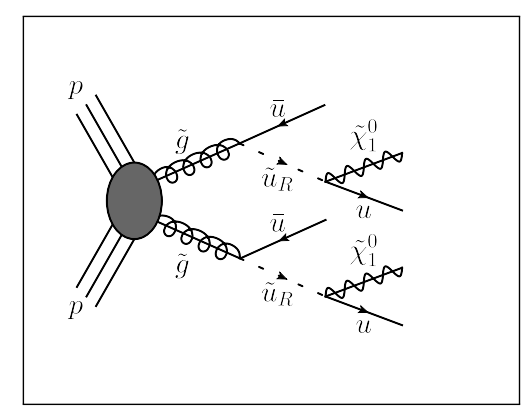

(a) $\tilde{g} \tilde{g}$

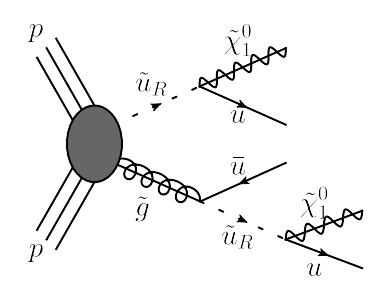

(b) $\tilde{g} \tilde{q}$
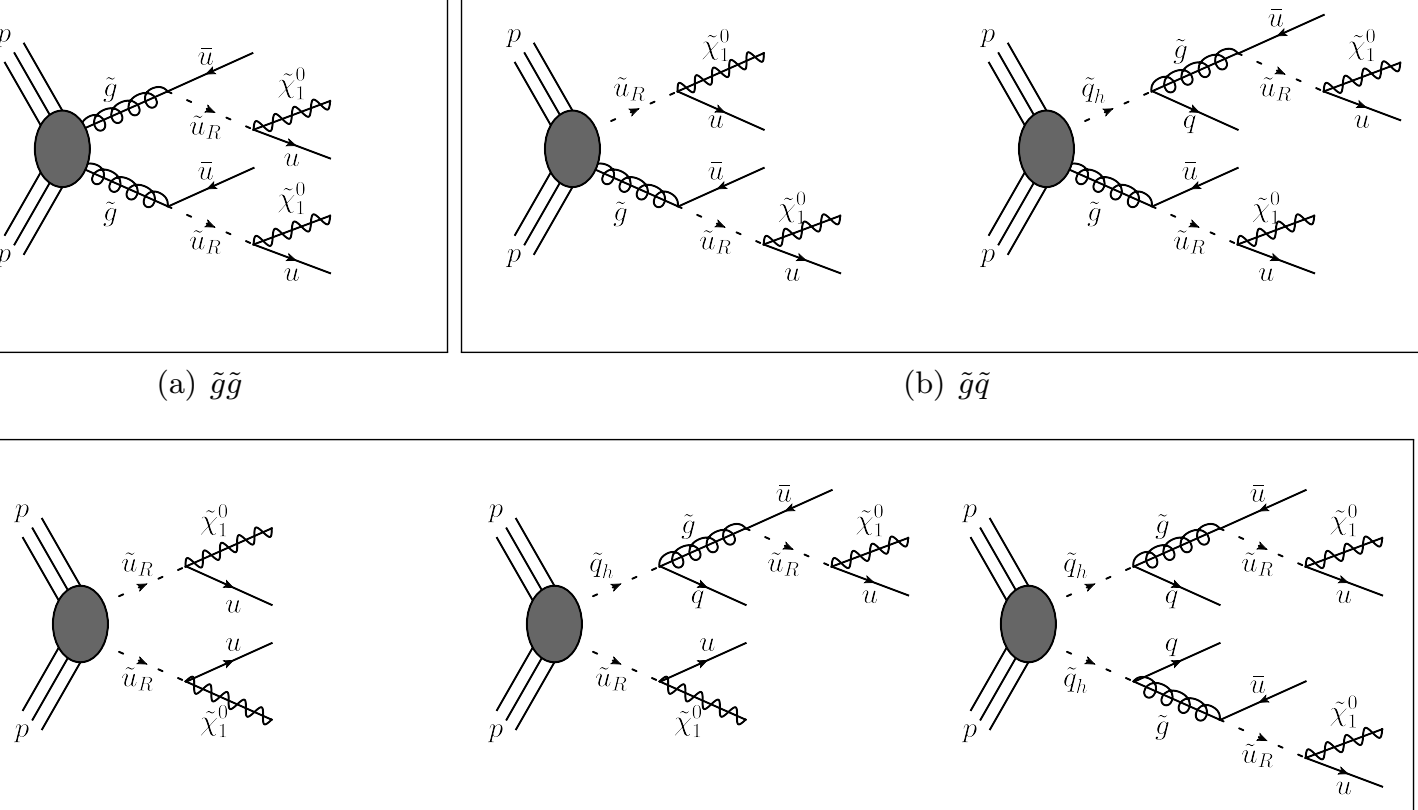

(c) $\tilde{q} \tilde{q}$

Figure 11. Representative Feynman diagrams for (a) $\tilde{g} \tilde{g}$, (b) $\tilde{g} \tilde{q}$ and (c) $\tilde{q} \tilde{q}$ production. All decays presented in this figure are implemented at the level of the parton shower programme.

scalar sum of the transverse momenta of all reconstructed jets, must be larger than $300 \mathrm{GeV}$. Thirdly, the missing transverse hadronic energy $H_{T}^{\text {miss }}=\left|\vec{H}_{T}^{\text {miss }}\right|$, with $\vec{H}_{T}^{\text {miss }}$ the negative of the vector sum of the transverse momenta of all reconstructed jets with a pseudorapidity $|\eta|<5$, must be larger than $300 \mathrm{GeV}$. Finally, the two leading jets and $\vec{H}_{T}^{\text {miss }}$ are imposed to be well separated in azimuth, $\Delta \phi\left(H_{T}^{\text {miss }}, j_{1,2}\right)>0.5$. When a third and a fourth jet are within the acceptance defined before, we additionally impose $\Delta \phi\left(H_{T}^{\text {miss }}, j_{3,4}\right)>0.4$.

Total cross sections with $\left(\sigma_{\text {fiducial }}\right)$ and without $\left(\sigma_{\text {inclusive }}\right)$ the above cuts at the $13 \mathrm{TeV}$ LHC are presented in table 6, for each of the six STR procedures listed in table 2. Gluinopair production leads to the smallest cross sections, as a result of the large gluino mass and the correspondingly small gluon PDF at large Bjorken $x$ 's. The NLO inclusive (fiducial) cross section turns out to be equal to about $0.33 \mathrm{fb}(0.23 \mathrm{fb})$, with a large $K$-factor of about 1.8 both for the inclusive and fiducial cases. This large $K$-factor originates from the large colour charge associated with the gluino, and the purely strong nature of the Born process, as was already discussed in section 4 . Scale uncertainties are reduced by a factor of two with respect to the LO ones and, as a consequence of the typically large Bjorken $x$ values associated with the production of a pair of $2 \mathrm{TeV}$ gluinos, the theoretical error is dominated by the PDF uncertainties. Both scale and PDF errors are essentially independent of the STR procedure adopted, which is the reason why we only report them for the istr=2 case. As far as the STR dependence itself is concerned, it is about $3 \%$, and thus much smaller than the other theoretical uncertainties. As is well known, this is an observable-dependent statement, and we shall show later that at the differential level things are more involved. 


\begin{tabular}{|c|c|c|c|c|c|c|c|c|}
\hline & {$[\mathrm{fb}]$} & & 2 & 3 & 4 & 5 & 6 & LO \\
\hline \multirow{2}{*}{$\tilde{g} \tilde{g}$} & $\sigma_{\text {inclusive }}$ & 0.331 & $0.330_{-18 \%}^{+19 \%} \pm 28 \%$ & 0.327 & 0.322 & 0.330 & 0.330 & $0.187_{-29 \%}^{+44 \%} \pm 27 \%$ \\
\cline { 2 - 8 } & $\sigma_{\text {fiducial }}$ & 0.228 & $0.227_{-18 \%}^{+19 \%} \pm 28 \%$ & 0.225 & 0.222 & 0.228 & 0.227 & $0.128_{-29 \%}^{+44 \%} \pm 27 \%$ \\
\hline \multirow{2}{*}{$\tilde{g} \tilde{q}$} & $\sigma_{\text {inclusive }}$ & 8.42 & $8.39_{-14 \%}^{+12 \%} \pm 6.9 \%$ & 8.38 & 8.35 & 8.41 & 8.40 & $5.49_{-25 \%}^{+38 \%} \pm 7.0 \%$ \\
\cline { 2 - 8 } & $\sigma_{\text {fiducial }}$ & 5.93 & $5.91_{-14 \%}^{+12 \%} \pm 6.9 \%$ & 5.90 & 5.87 & 5.93 & 5.92 & $3.86_{-26 \%}^{+38 \%} \pm 7.0 \%$ \\
\hline \multirow{2}{*}{$\tilde{q} \tilde{q}$} & $\sigma_{\text {inclusive }}$ & 20.4 & $20.4_{-10 \%}^{+7.8 \%} \pm 2.2 \%$ & 20.4 & 20.4 & 20.4 & 20.4 & $14.9_{-22 \%}^{+30 \%} \pm 2.2 \%$ \\
\cline { 2 - 8 } & $\sigma_{\text {fiducial }}$ & 14.8 & $14.8_{-9.9 \%}^{+7.8 \%} \pm 2.2 \%$ & 14.8 & 14.7 & 14.8 & 14.8 & $10.8_{-21 \%}^{+30 \%} \pm 2.2 \%$ \\
\hline
\end{tabular}

Table 6. Total inclusive and fiducial cross sections (in $\mathrm{fb}$ ) at the $\sqrt{S}=13 \mathrm{TeV}$ LHC. The leftmost errors stem from scale variations, the rightmost ones from PDF uncertainties. We have set str_include_pdf=True and str_include_flux=True (see section 5.3).

Finally, we remark that the Monte Carlo integration errors are equal to about $0.2 \%$, and have therefore been ignored.

Because of the smaller $\tilde{u}_{R}$ mass, the cross sections of gluino-squark associated production and of squark pairs are 25 to 60 times larger than the gluino-pair ones. This behaviour is driven by that of the parton luminosities: valence quarks contribute significantly, since one is in an $x$-region where their PDFs are large. Correspondingly, the PDF uncertainties are much smaller than for gluino-pair production, as deep-inelastic scattering, fixed-target experiment data, LHC Drell-Yan, forward $W$-boson, and $Z$-boson data allow one to strongly constrain the fit of the valence quark densities at large $x$ 's. This implies that for these processes, at variance with the case of gluino pairs, the PDF errors are smaller than the scale ones, in spite of the fact that the latter are a factor of three smaller at the NLO than at the LO (as opposed to a factor of two for gluino-pair production). As far as the STR-option dependence is concerned, it is below $1 \%$ and thus, as in the case of the gluinos, largely subdominant with respect to the other uncertainties.

In figures 12, 13, and 14 we present six differential distributions for gluino-pair, gluinosquark, and squark-pair production, respectively. Such observables are directly relevant to the CMS SUSY search of ref. [236], and thus all results are obtained by applying the fiducialvolume cuts defined before. Each panel of each figure has the same layout, namely: the main frame presents the differential distributions in $\mathrm{pb} / \mathrm{GeV}$ or $\mathrm{pb}$, at the $\mathrm{LO}$ (dashed black histogram) and at the NLO (solid coloured histograms; there are six of these, corresponding to the six STR options we have considered). The statistical errors are shown as error bars, while the scale uncertainties are displayed as dark-blue bands; finally, the linear sums of the scale and PDF errors are represented as light-blue bands. The lower inset presents the ratios of the six NLO predictions over the LO one, the latter evaluated with the central scale choice and central PDF (i.e. these are the standard $K$ factors).

Mirroring what has been found for total rates, PDF uncertainties are quite substantial for gluino-pair production, as one is always in a kinematic regime where large Bjorken $x$ 's 

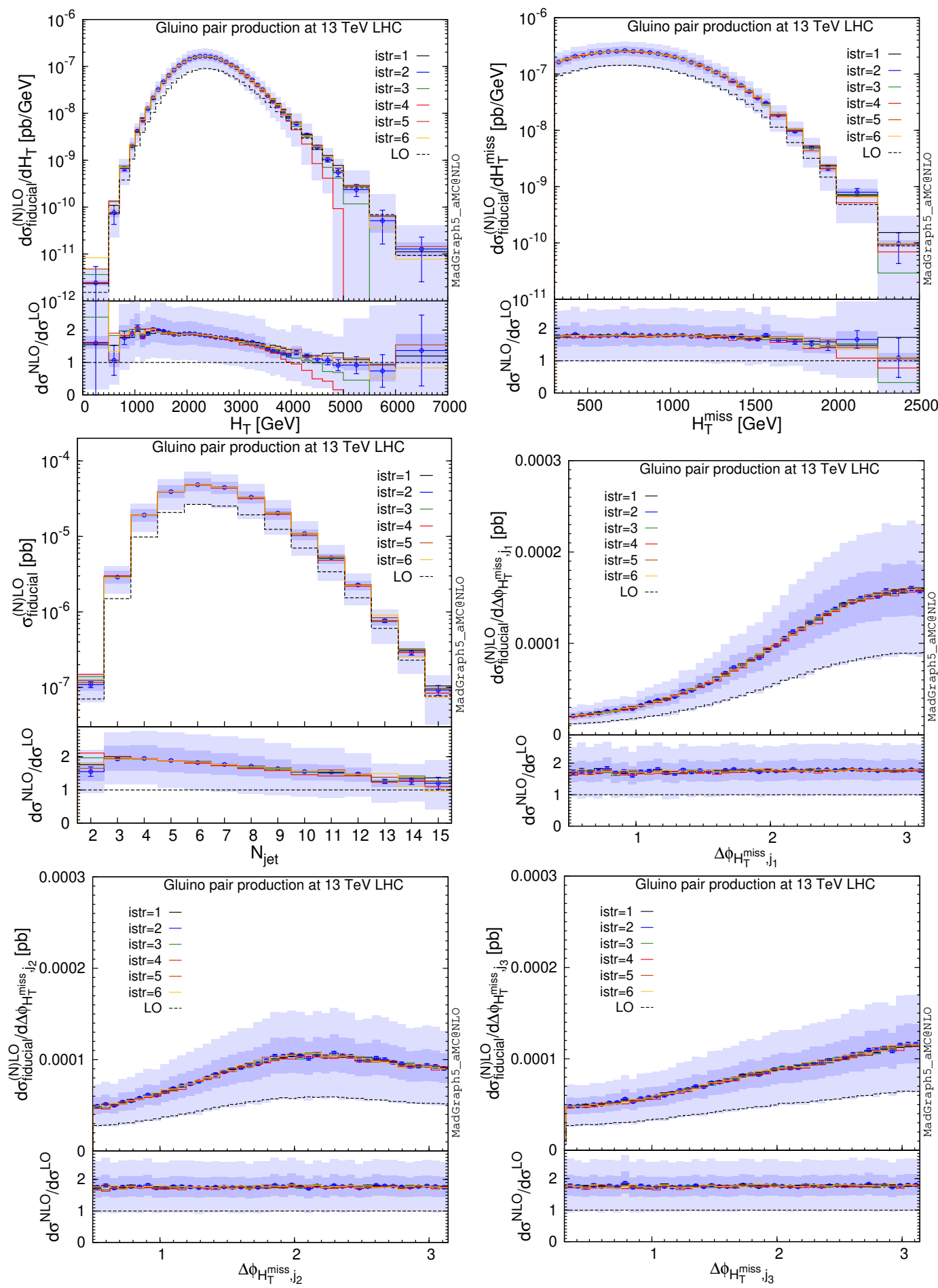

Figure 12. Representative differential distributions for gluino-pair production at $\sqrt{S}=13 \mathrm{TeV}$ LHC, relevant to the CMS analysis of ref. [236]. We consider the $H_{T}$ (upper left), $H_{T}^{\text {miss }}$ (upper right) and jet multiplicity (centre left) spectra, as well as the azimuthal separation between the $H_{T}^{\text {miss }}$ vector and the three hardest jets (centre right and bottom panels). The error bars represent the Monte Carlo integration errors in the default istr $=2$ choice. The NLO results are obtained with str_include_pdf=True and str_include_flux=True. 

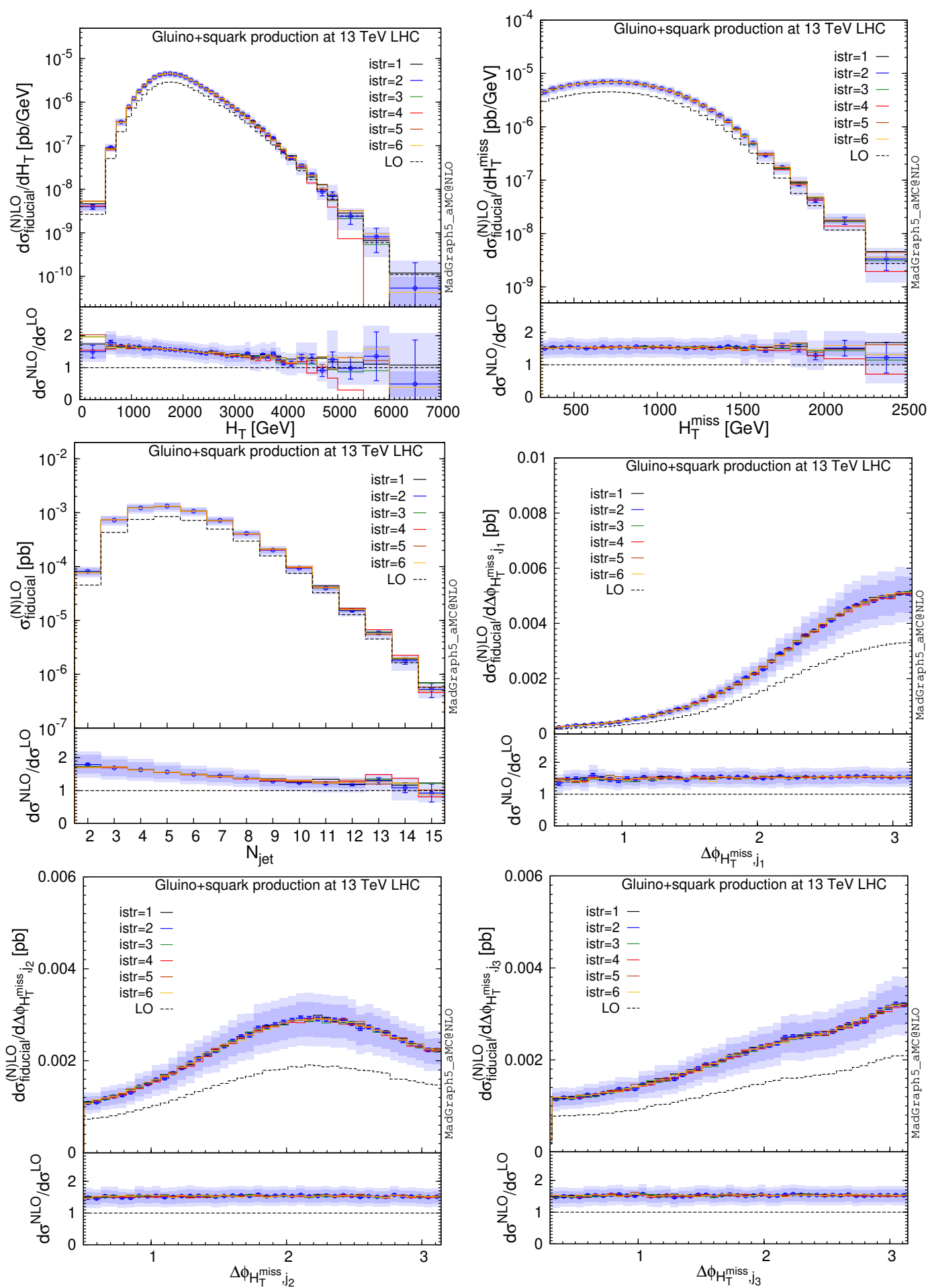

Figure 13. Same as in figure 12 but for gluino-squark production. 

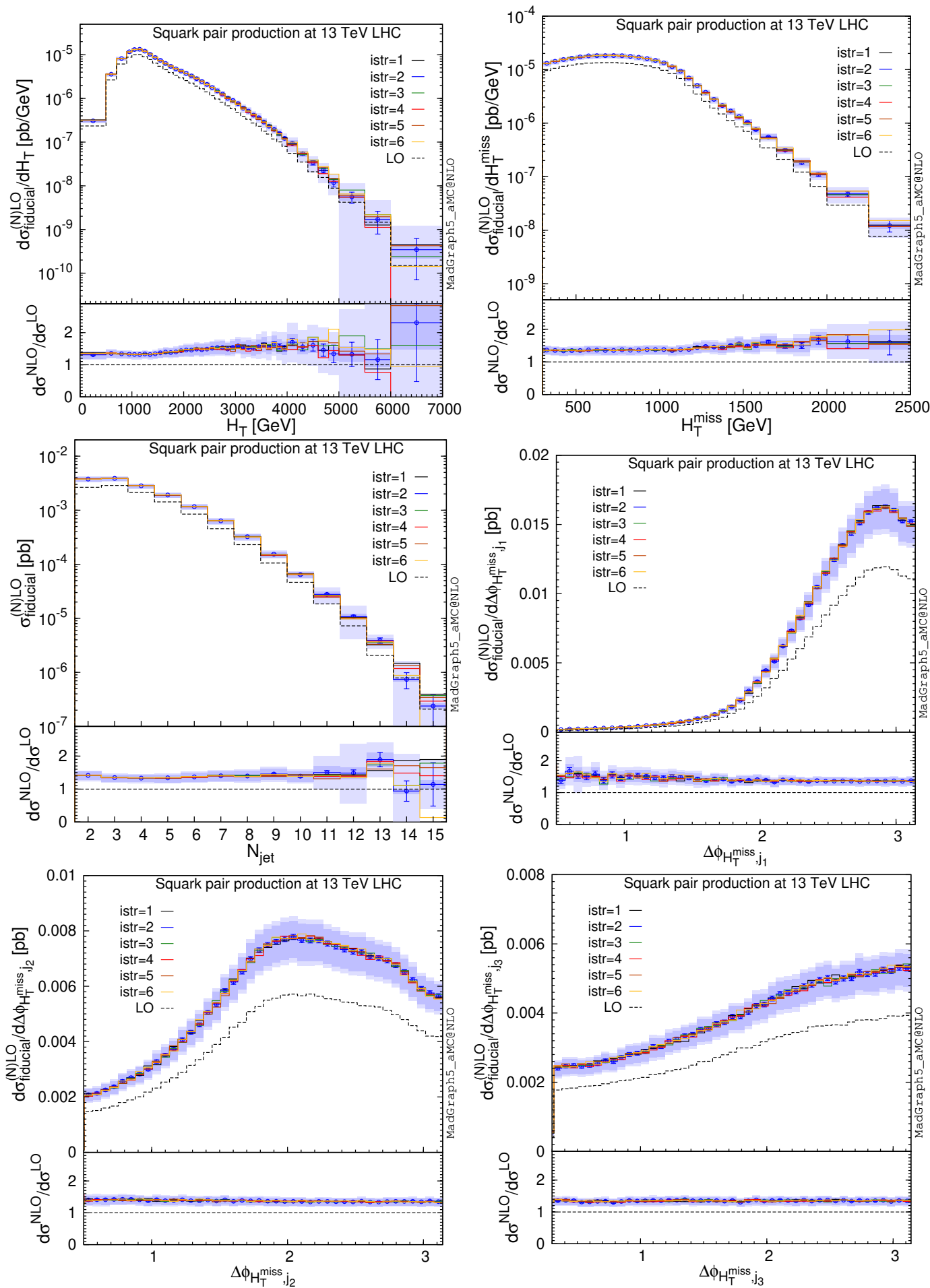

Figure 14. Same as in figure 12 but for squark-squark production. 
are relevant. For the other two processes, such uncertainties are reduced, except in those phase-space corners where again one is sensitive to large Bjorken $x$ 's values, e.g. for large $H_{T}$ or $H_{T}^{\text {miss }}$. It is therefore obvious that, despite the progress made in the computations of the short-distance cross sections, precise simulations at large scales can only be achieved by constraining much more strongly the PDFs at $x \rightarrow 1$. As far as the $K$-factors are concerned, they are found to be relatively flat for most observables, and close to 2 for $\tilde{g} \tilde{g}$ production, to 1.5 for $\tilde{g} \tilde{q}$ production, and to 1.4 for $\tilde{q} \tilde{q}$ production, respectively. Distinctly non-flat $K$ factors are obtained in particular in the cases of the $H_{T}$ and jet-multiplicity distributions. This helps to stress the fact that, in general, the re-scaling of LO+PS predictions by an overall constant factor is a dangerous procedure that may lead to unreliable results, which underscores one of the main motivations of the present paper.

It is interesting to observe that the dependence on the STR choice is generally very mild also at the differential level, which gives one confidence on the description of multijet SUSY-induced signatures through the production-and-decay picture of eqs. (6.2)-(6.4). A notable exception is the large- $H_{T}$ region (for all of the three processes, although it is particularly prominent in the case of gluino-pair production), where the STR dependence becomes extremely large, and appears to be pathological. We shall argue that, in fact, this behaviour allows the method to self-diagnose that it is being applied in regions where its founding assumptions are dubious at best. Let us start with a technical explanation. The STR procedures that differ the most from the median are those associated with istr $=3,4$ - these are DS procedures, option A (see table 2). In these cases, through a reshuffling operation the momenta of the incoming partons are changed, thereby implying that the corresponding Bjorken $x$ 's are changed too. This affects the value of both the flux factor and the PDFs. In the notation of section 5.2:

$$
f\left(m_{\delta \gamma}^{2}\right) \mathbb{P}\left(\left|\mathcal{A}_{a b \rightarrow \delta \gamma X}^{(\beta)}\right|^{2} \mathrm{~d} \phi\right) \sim \frac{x_{a} x_{b}}{\bar{x}_{a} \bar{x}_{b}} \frac{f_{a}^{\left(H_{1}\right)}\left(\bar{x}_{a}\right) f_{b}^{\left(H_{2}\right)}\left(\bar{x}_{b}\right)}{f_{a}^{\left(H_{1}\right)}\left(x_{a}\right) f_{b}^{\left(H_{2}\right)}\left(x_{b}\right)},
$$

which shows the effect on the flux and parton-luminosity factors (first and second terms on the r.h.s. of eq. (6.5), respectively) at the level of the subtraction cross section (last term on the r.h.s. of eq. (5.18)). If one generates a far off-shell kinematic configuration, $m_{\delta \gamma} \gg$ $m_{\beta}$, then $x_{a} x_{b} \gg \bar{x}_{a} \bar{x}_{b}$. Therefore, since eventually all PDFs decrease with increasing Bjorken $x$ 's, both terms on the right-hand side of eq. (6.5) are large, which implies a strong suppression of the physical cross section of eq. (5.18) in this kinematic region (since the subtraction term is large); so strong, in fact, that it may become negative. Owing to the structure of the function $f\left(\mathrm{~m}^{2}\right)$ given in eq. (5.31), this feature is more pronounced for istr $=4$ than for istr $=3$, which clearly shows in the figures.

In fact, although the mechanism we have just described is responsible for the behaviour of the cross sections in the large- $H_{T}$ region, its effects are particularly dramatic in gluinopair production owing to $x_{a, b} \simeq 1$ there (in other words, if the gluino had a smaller mass, the subtraction cross section would be significantly smaller). For such Bjorken $x$ 's, the central values of the PDFs are very suppressed and poorly constrained, and thus affected by very large residual uncertainties: indeed, we see from figures $12-14$ that as soon as the STR-choice dependence becomes very large, so does the PDF uncertainty, the effect being 
larger when parton luminosities involving the gluon density are relevant. We have verified that, by "removing" the luminosity factor from eq. (6.5) by setting str_include_pdf equal to False (see section 5.3), the results obtained with istr $=3,4$ are much closer to the others, thus showing that the large STR-choice dependence is driven by the PDFs.

We point out that sizeable differences due to STR options should be expected also for $m_{\delta \gamma} \ll m_{\beta}$. However, while the Breit-Wigner distribution is symmetric around $m_{\beta}$, the flux and PDF ratios are not. This implies that the explanation given above may not apply in this kinematic regime. However, regardless of which mechanism is responsible for an enhanced STR-choice dependence, the take-home message is the following: such a dependence is the signal that describing the process of interest by means of a cross section dominated by resonant production of (s)particles which subsequently decay is simply not adequate, and a full (unfortunately, more complicated) computation is required. Ultimately, the decision of where to stop trusting a simplified computation rests with the user, and it depends on many factors, in particular on whether one is interested in a class 1 or class 2 approach (see the itemised list below eq. (5.10) in section 5). It is because of this that MG5_AMC does not set to zero the subtraction term in eq. (5.18) when far off-shell: if needed, such a cut must be implemented at the analysis level.

\section{Conclusions}

With the steady increase of the statistics accumulated by the LHC experiments, and the absence of positive results in the searches for new physics, it becomes necessary to improve the accuracy of the simulations of BSM signals, thus matching that of their SM backgrounds. By far and large, this currently means matrix elements computed at the NLO in QCD matched to parton showers (NLO+PS). Such a necessity has spurred some recent theoretical activity, whereby authors have addressed the needs of specific search strategies.

The goal of this paper has been that of rendering such an improvement systematic (i.e. achievable for arbitrary processes and for a vast class of renormalisable theories). This is feasible thanks to the powerful and flexible environment constituted by the automated programme MADGRAPH5_aMC@NLO and the physics models it can use for simulations. In particular, for the sake of the present work, two major limitations have been lifted. Firstly, at the level of the construction of models, we have overhauled the way in which FEYnRuLES and NLOCT deal with on-shell renormalisation schemes, so that a much larger flexibility is achieved, that helps deal with the difficult cases especially relevant to supersymmetric theories. Secondly, at the level of the MAdGraPh5_aMC@NLO code proper, we have automated a variety of solutions to the problem posed by the presence of partonic channels that appear beyond the leading order in perturbative computations, and that feature narrow resonances; this problem is particularly acute in theories with a complex mass spectrum. Such solutions, that we have dubbed Simplified Treatments of Resonances (STR), generalise the so-called on-shell subtractions, and encompass the Diagram Removal (DR) and Diagram Subtraction (DS) strategies introduced in the last few years in the context of NLO+PS simulations. Technically, these two pieces of work have been implemented, 
respectively, in a plugin for FEynRules, called MoGRe, and in a plugin for MG5_AMC, called MADSTR.

As a proof-of-concept, we have generated an NLO UFO model for the MSSM with a widely-used renormalisation scheme, and we have studied processes that feature intermediate resonances at the LHC, at the NLO+PS accuracy and with a realistic set of final-state cuts. We have performed thorough self-consistency checks of our implementation, and compared some loop matrix elements generated by the code with those resulting from analytical computations. It is important to bear in mind that the core structure of the MADGRAPH5_aMC@NLO code, which has been only minimally affected by the present work, has by now been very extensively validated in countless simulations. As a further a posteriori validation, we have compared total-rate leading- and next-to-leading order results for benchmark $2 \rightarrow 2$ processes with those of the public codes Prospino2 and REsummino, with the restrictions that these two programmes enforce. Such comparisons, whose details can be found in section 3, in some cases show disagreements (of different origins) among the various predictions, and further underscore the advantages of a general, process-independent, and automated implementation.

In conclusion, with the present work we have achieved, for the first time, the complete automation of NLO+PS simulations for supersymmetric-particle production at hadron colliders in the framework of the MSSM with a generic particle spectrum, and set up the tools for dealing with similarly involved theories by means of a user-driven framework. We point out, however, that we have not yet implemented and tested the general solution, introduced in ref. [70], to the problem posed by unstable resonances in the context of the complexmass scheme [237, 238]. Such a solution requires further developments in FeynRules and NLOCT, so that NLO UFO models contain the necessary routines for dynamically and automatically selecting, according to the particle spectrum, the appropriate Riemann sheets for the calculation of the UV counterterms. This is left to future work.

All of the computer programmes relevant to this paper are publicly available - on top of MadGraph5_aMC@NLO, the MSSM model, together with the MoGRE plugin and ready-to-be-used Mathematica notebooks, can be found at http://feynrules.irmp. ucl.ac.be/wiki/MSSMatNLO, while the MADSTR plugin can be downloaded from https: //code.launchpad.net/ maddevelopers/mg5amcnlo/MadSTRPlugin.

This paper is accompanied by ancillary files, stored on the electronic archive, that collect NLO QCD results for total rates of pair-production supersymmetric processes, which we have refrained from including here for reasons of space. 


\section{Acknowledgments}

We would like to thank Dorival Goncalves and David Lopez-Val for collaboration at early stages of the development of MADSTR and for discussions on related topics. We also thank Davide Pagani for discussions on these topics. BF and HSS are supported by the LABEX ILP (ANR-11-IDEX-0004-02, ANR-10-LABX-63). KM is supported by JSPS KAKENHI Grant No. 18K03648. MPAS is supported by the BMBF under contract 05H18PMCC1 and the DFG through the Research Training Network 2149 "Strong and weak interactions from hadrons to dark matter". MZ is supported by the Netherlands National Organisation for Scientific Research (NWO). SF is grateful to the CERN TH division for the hospitality during the course of this work. VH is supported by the European Research Council (ERC) grant No 694712 (PertQCD), and by the Swiss National Science Foundation (SNSF) grant No 179016.

\section{A Conventions for one-point and two-point functions}

In the analytical formulas presented in this paper, all $A$ and $B$ loop-integrals have been normalised as

$A_{0}\left(m^{2}\right)=\int \frac{\mathrm{d}^{D} q}{i \pi^{2}} \frac{\left(2 \pi \mu_{R}\right)^{2 \epsilon}}{\left[q^{2}-m^{2}\right]}$ and $B_{\{0, \mu\}}\left(p^{2} ; m_{1}^{2}, m_{2}^{2}\right)=\int \frac{\mathrm{d}^{D} q}{i \pi^{2}} \frac{\left(2 \pi \mu_{R}\right)^{2 \epsilon}\left\{1, q_{\mu}\right\}}{\left[q^{2}-m_{1}^{2}\right]\left[(q+p)^{2}-m_{2}^{2}\right]}$,

where we recall that $D=4-2 \epsilon$ is the number of spacetime dimensions and $\mu_{R}$ is the regularisation scale (taken equal to the renormalisation scale). The $B_{\mu}$ vectorial integral has been further reduced to a scalar integral using Lorentz covariance,

$$
B_{\mu}\left(p^{2} ; m_{1}^{2}, m_{2}^{2}\right)=p_{\mu} B_{1}\left(p^{2} ; m_{1}^{2}, m_{2}^{2}\right),
$$

and the $B_{1}$ integral is connected to several $B_{0}$ integrals as

$$
B_{1}\left(p^{2}, m_{1}^{2}, m_{2}^{2}\right)=-\frac{1}{2} B_{0}\left(p^{2} ; m_{1}^{2}, m_{2}^{2}\right)+\frac{m_{2}^{2}-m_{1}^{2}}{2 p^{2}}\left[B_{0}\left(p^{2} ; m_{1}^{2}, m_{2}^{2}\right)-B_{0}\left(0 ; m_{1}^{2}, m_{2}^{2}\right)\right],
$$

the $p^{2} \rightarrow 0$ limiting case involving a derivative of the $B_{0}$ function with respect to the $p^{2}$ variable instead of the squared bracket. Explicitly, one gets

$$
\begin{aligned}
A_{0}\left(m^{2}\right) & =m^{2}\left[\frac{1}{\bar{\epsilon}}+1+\log \frac{\mu_{R}^{2}}{m^{2}}\right], \\
B_{0}\left(p^{2} ; m_{1}^{2}, m_{2}^{2}\right) & =\frac{1}{\bar{\epsilon}}+2-\log \frac{p^{2}}{\mu_{R}^{2}}+\sum_{i=1}^{2}\left[\gamma_{i} \log \frac{\gamma_{i}-1}{\gamma_{i}}-\log \left(\gamma_{i}-1\right)\right],
\end{aligned}
$$

where

$$
\gamma_{1,2}=\frac{p^{2}-m_{2}^{2}+m_{1}^{2} \pm \sqrt{\left(p^{2}-m_{2}^{2}+m_{1}^{2}\right)^{2}-4 p^{2} m_{1}^{2}}}{2 p^{2}}
$$

and with the ultraviolet-divergent parts of the integrals being written in terms of the number of spacetime dimensions and the Euler-Mascheroni constant $\gamma_{E}, \frac{1}{\bar{\epsilon}}=\frac{1}{\epsilon}-\gamma_{E}+\log 4 \pi$. 
Several special limits for the $B_{0}$ function and its $B_{0}^{\prime}$ derivative are useful,

$$
\begin{aligned}
& B_{0}\left(0, m^{2}, m^{2}\right)=\frac{1}{\bar{\epsilon}}+\log \frac{\mu_{R}^{2}}{m^{2}}, \\
& B_{0}\left(0, m_{1}^{2}, m_{2}^{2}\right)=\frac{1}{\bar{\epsilon}}+1+\frac{m_{1}^{2} \log \frac{\mu_{R}^{2}}{m_{1}^{2}}-m_{2}^{2} \log \frac{\mu_{R}^{2}}{m_{2}^{2}}}{m_{1}^{2}-m_{2}^{2}}, \\
& B_{0}\left(m^{2}, m^{2}, 0\right)=\frac{1}{\bar{\epsilon}}+2+\log \frac{\mu_{R}^{2}}{m^{2}}, \\
& B_{0}^{\prime}\left(0, m^{2}, m^{2}\right)=\frac{1}{6 m^{2}}, \\
& B_{0}^{\prime}\left(0, m_{1}^{2}, m_{2}^{2}\right)=\frac{m_{1}^{2}+m_{2}^{2}}{2\left(m_{1}^{2}-m_{2}^{2}\right)^{2}}+\frac{m_{1}^{2} m_{2}^{2}}{\left(m_{1}^{2}-m_{2}^{2}\right)^{3}} \log \frac{m_{2}^{2}}{m_{1}^{2}}, \\
& B_{0}^{\prime}\left(m^{2}, m^{2}, 0\right)=-\frac{1}{2 m^{2}}\left[\frac{1}{\bar{\epsilon}}+2+\log \frac{\mu_{R}^{2}}{m^{2}}\right] .
\end{aligned}
$$

We stress that since this paper does not consider the complex-mass scheme, the renormalisation counterterms are defined using only the real part of the two-point functions.

\section{B The MoGRE package}

In order to circumvent the lack of flexibility concerning the choice of the renormalisation scheme in the current FeynRules release, we have developed a plugin, called MoGRe, that is fully flexible in the way a bare Lagrangian can be renormalised. This package can be downloaded from the wikipage collecting details about the MSSM at NLO implementation in FeynRules introduced in this paper, http://feynrules.irmp.ucl.ac.be/wiki/ MSSMatNLO.

In practice, the user starts by loading FeynRules and any given model implementation in the Mathematica session. The MoGRe plugin can then be imported as any Mathematica package, by typing,

\section{Begin ["MoGRe-"]; $<$ MoGRe.m; End [] ;}

where one assumes that the current directory is the one containing the plugin.

\section{B.1 The main method MoGRe\$Renormalize and its options}

The main function of the MoGRe plugin is called MoGRe\$Renormalize and takes a Lagrangian as an input, as for instance in the following example

MoGRe $\$$ Renormalize [LMSSM]

where LMSSM stands for the MSSM Lagrangian. The user is allowed to specify three options that modify the behaviour of the method and that respectively address the treatment of the four-scalar vertices, loop-induced field mixings and the nature of the interaction in 
which the loop corrections are evaluated. These options can be set following a standard Mathematica syntax, as for instance through the command

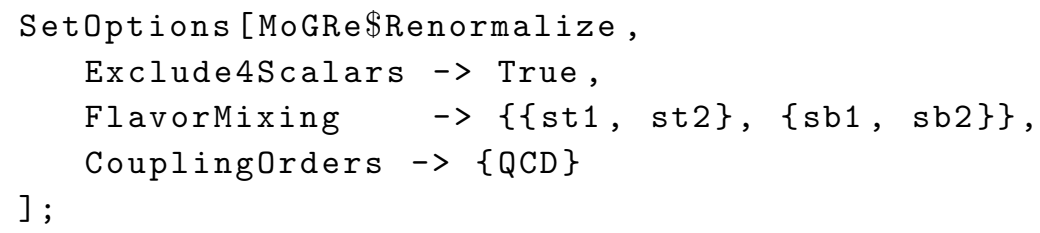

This first indicates, through the Exclude4Scalars option, that all model four-scalar interactions have to be ignored in the renormalisation procedure, so that the corresponding counterterms are not evaluated. The default choice for this option (set to True in our example) is False. While strictly speaking ignoring the renormalisation of the four-scalar vertices is incorrect, these vertices rarely appear at tree-level so that the associated counterterms are often not necessary. Avoiding their calculation and their inclusion in the final UFO model therefore allows for an increase of the efficiency of the computations, both at the NLOCT and MG5_AMC level.

Secondly, we provide information on the different sets of fields that mix at the oneloop level through the FlavorMixing option of the MoGRe\$Renormalize method. If set to True (the default choice), all fields carrying the same quantum numbers and lying in the same spin and colour representation are assumed to mix. In contrast, all loop-level mixings are forbidden if this option is set to False. The user has also the possibility, like in our example, to provide a list with the different sets of fields that mix at the one-loop level. Any physical field can be used in such a list, and the code further checks whether the input is compatible with the representation of the involved fields under the model gauge group. In our example, we have forbidden any loop-level mixing, except the one of the two stop-eigenstates (denoted st1 and st2 in the FeynRules model) and the one of the two sbottom-eigenstates (denoted sb1 and sb2 in the FeynRules model), as given by eq. (2.22).

Finally, the last option indicates which type of interaction should be renormalised, among all the interactions declared in the MR\$InteractionOrderHierarchy option of the FeynRules model implementation. In the MSSM implementation, two types of interactions are available, namely the QCD and QED ones. In the above example, that matches the physics goals of this paper (NLO QCD corrections for the MSSM), we have selected the QCD interaction type QCD.

Before describing how the bare Lagrangian is technically renormalised, we detail in the next subsections various methods that can be used to simplify the model and modify the way in which MoGRe\$Renormalize works.

\section{B.2 Simplifying the procedure}

In general, all external parameters have to be renormalised, which yields a heavy renormalisation procedure for complex models like the MSSM. However, some parameters may not need to be renormalised, like the electromagnetic coupling constant that does not receive any correction at one loop in QCD. Whilst this type of information can be useful to speed up the renormalisation procedure, the programme cannot guess it at this stage where no 
calculation has been done yet. For this reason, the user is allowed to declare the parameters that should not be renormalised with the MoGRe`DefineUnrenormalizedParameters method that takes as arguments the corresponding symbols as implemented in the FEYNRules model. The arguments can also be provided as a unique list. Going back to the considered example where only QCD corrections matter, the command

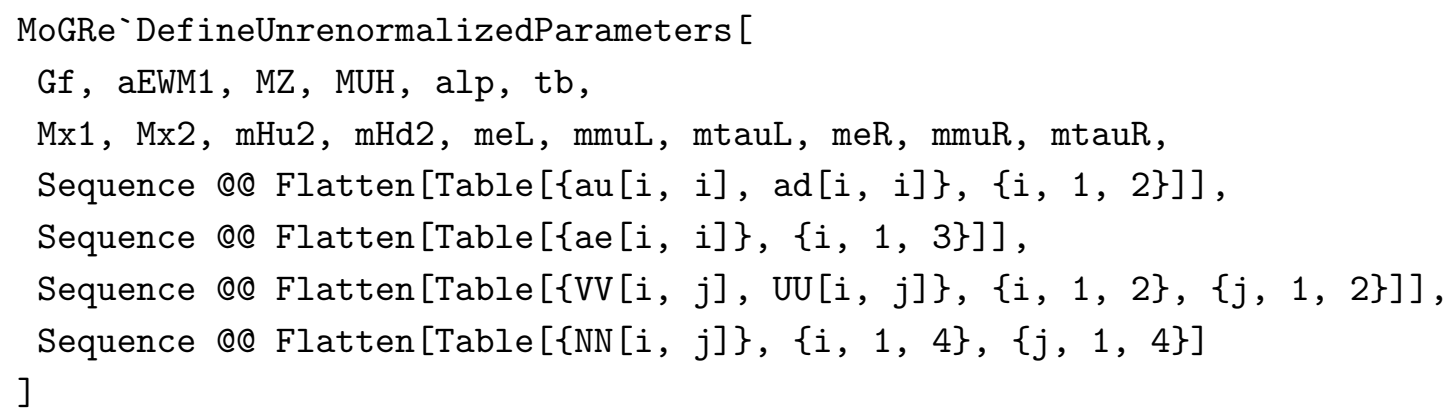

leads to declaring all parameters connected to the electroweak sector (namely the electroweak inputs, the Higgs sector parameters, the electroweak gaugino and scalar soft masses as well as the chargino and neutralino mixing matrices) to be insensitive to QCD corrections at one loop. The renormalisation of all relevant internal parameters is accordingly and automatically simplified through their functional dependence on the above parameters.

Similarly, the code assumes by default that all fields get renormalised, although this may not be the case in practice. For instance, the weak boson two-point functions are insensitive to QCD corrections at one loop. This type of information can be passed to the code by means of the MoGRe`DeclareUnrenormalizedFields method that takes as arguments all fields that should not be renormalised. The arguments can be provided either sequentially or under the form of a list. For instance in the MSSM implementation worked out in this paper, we could use

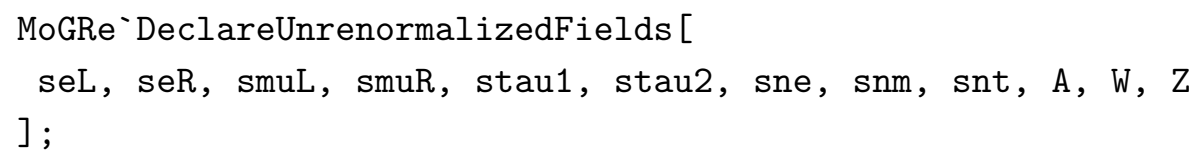

although the sleptons are in principle not necessary as they do not appear in any QCD vertex (but we keep them here for illustrative purposes). This prevents all charged sleptons, sneutrinos and electroweak bosons from being renormalised. All remaining fields will be renormalised, the associated wave-function renormalisation constants being taken complex by default. Reality conditions can be enforced through the usage of the MoGRe`RealFieldRenormalisation method, that takes as an argument the symbols associated with the concerned fields. All relevant symbols can be given again either under the form of a list or of a sequence. In the case where no argument is provided, all wave-function renormalisation constants are taken real, as with the following example,

\section{MoGRe`RealFieldRenormalisation [ ]}

For mixing fields for which matrix renormalisation is in order, the method acts on all the elements of the renormalisation matrix. 


\section{B.3 Restrictions}

The FeynRules model implementation may contain (external or internal) parameters that are numerically vanishing when default values are accounted for. While it is in general safer to keep these parameters all along the renormalisation procedure, so that they are renormalised and get associated with potentially non-vanishing renormalisation constants, this is often not necessary and only leads to heavier subsequent calculations. The MoGRE package by default takes care of the removal of these zero parameters both from the treelevel Lagrangian and from the rules dedicated to the replacement of the bare quantities by the renormalised ones. In this way, those parameters will not be renormalised as not present in the bare Lagrangian anymore, and the code will make sure that they do not re-appear through the renormalisation of other quantities. For instance, the CKM matrix is often taken diagonal, so that MoGRe by default removes all its off-diagonal elements. This behaviour can be turned off by issuing the command

EnforceZeros = False;

\section{B.4 Specifying the renormalisation scheme}

As detailed in section 2.2.4, there is no unique way to define an OS renormalisation scheme in the MSSM and in SUSY in general. This consisted in the main reason that has led to the development of the MoGRe package. Scheme-specific renormalisation conditions can be added by the user by making use of a dedicated method named AddRenormalizationCondition. The latter takes two arguments, a renormalisation constant (associated with either a parameter or a field) and a function of different parameters, fields and other renormalisation constants. As a result, the first renormalisation constant will be considered equal to the function given in the second argument. The way in which field renormalisation constants should be input follows the FEYNRuLES syntax, wave-function renormalisation constants being provided as

$$
\begin{aligned}
& \text { FR } \$ d \operatorname{deltaZ}[\{f l d 1, f l d 2\},\{\{\}\}]] \\
& \text { FR } \$ \operatorname{deltaZ}[\{f l d 1, f l d 2\},\{\{" L "\}\}]]
\end{aligned}
$$

FR\$deltaZ $[\{f l d 1, f l d 2\},\{\{" R "\}\}]]$

for non-fermionic, left-handed fermionic and right-handed fermionic fields respectively. In the diagonal case, the two field symbols $f l d 1$ and $f l d 2$ are equal. In a non-diagonal field mixing case, they can be different. For a parameter prm, the corresponding syntax reads

FR\$delta $[\{$ prm\}, \{\}] ]

For instance, the stop mixing angle conditions of eq. (2.36) could be implemented as

$$
\begin{aligned}
& \text { AddRenormalizationCondition[FR\$delta[\{Rtop }[1,1]\},\{\}], 1 / 4 \text { Rtop }[2,1] * \\
& \text { ( FR\$deltaZ }[\{\text { st1,st2\},\{\{\}\}] - Conjugate }[F R \$ d e l t a Z[\{\text { st2,st1\},\{\{\}\}]] )]; }
\end{aligned}
$$

where the Rtop symbol represents the stop mixing matrix $S_{\tilde{t}}$ of eq. (2.18) in our FeynRuLES implementation. This is equivalent to indicating to the MoGRE package that

$$
\left(\delta S_{\tilde{t}}\right)_{11}=-s_{\tilde{t}} \delta \theta_{\tilde{t}}=\frac{1}{4}\left(S_{\tilde{t}}\right)_{21}\left[\delta Z_{\tilde{t}, 12}-\delta Z_{\tilde{t}, 21}^{*}\right] .
$$




\section{B.5 Clearing a renormalisation scheme}

All the options detailed above can be reset by calling the MoGRe`ClearRenormalizationScheme [] method.

\section{B.6 Technical details on the functioning of the method}

\section{B.6.1 Initialization}

The MoGRe\$Renormalize method begins with a check that all parameters passed as options are meaningful. Appropriate error messages are printed to the screen if necessary.

In a second step, still prior to any computation, simplifications are performed and the Lagrangian is put under an internal format allowing for a more efficient run. More precisely, the Lagrangian is truncated from its constant terms, and all parameters that are vanishing are removed except if the EnforceZeros flag has been set to False (see section B.3). The Lagrangian is then expanded so that all (unphysical) gauge eigenstates are replaced by physical mass eigenstates. In the case where four-scalar interactions are requested not to be renormalised (see section B.1), they are removed from the Lagrangian. They will however be reintroduced at the very end of the renormalisation procedure, as those interactions could appear into given loop diagrams. Moreover, MoGRE requires that all tree-level bilinear terms are canonically normalised and that all kinetic and mass mixings have been appropriately rotated away by the user. In practice, the code ignores all bilinear terms provided by the user and reintroduces the canonical ones directly on the basis of the model field content.

As a last initialisation step, MoGRe makes use of the FeynRules model information on the external and internal parameters that must be exchanged during the renormalisation procedure (the FeynRules FR\$LoopSwitches option [58]). In the FeynRules syntax, FR \$LoopSwitches consists in a list of 2-tuples of parameters,

FR $\$$ LoopSwitches $=\{\{$ prm1 $\operatorname{prm} 2\}, \ldots\}$

in which prm1 is external and prm2 is internal. Prior to the renormalisation of the model, prm2 is made external and prm1 internal, the dependence of this last parameter on the other parameters being derived by the code. For instance, in many publicly available FEYNRules models, the $W$-boson mass $m_{W}$ is derived from the other electroweak inputs. OS renormalisation however requires $m_{W}$ to be external. The FR\$LoopSwitches is then used to trade it, for example, with the Fermi constant $G_{F}$. We refer to the FeynRules manual [58] for more information.

Some parameters can moreover be doubly defined in FeynRules models, like the Yukawa couplings and the fermion masses in the SM that are taken as different input parameters, although being numerically equal. The idea behind this trick consists in allowing for massless fermions and non-zero Yukawa couplings at tree-level. However, this makes the theory ill-defined when renormalisation is at stake, as all these parameters must be enforced to be equal when counterterms are evaluated. This can be achieved by means 
of the FR \$RmDblExt FeynRules parameter, that consists in a list of Mathematica replacement rules mapping one of the doubly-defined parameters to the other. For instance,

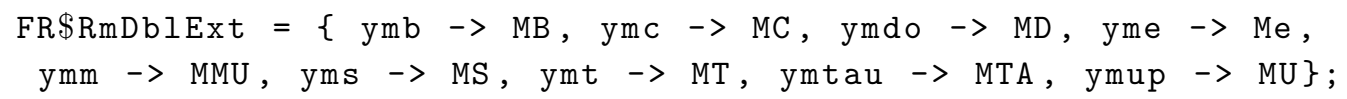

replaces every single Yukawa coupling (normalised to be exactly equal to the associated mass parameter) by the corresponding fermion mass. Such a replacement is also enforced in MoGRe in the case where the FR\$RmblExt parameter exists. Concerning the model introduced in this work, all parameters are uniquely defined so that this is irrelevant.

Along these lines, we recall that we rely on NLOCT [59] for the analytical computation of the various counterterms. This implies that care must be taken with any coupling depending on particle masses, like the Yukawa couplings or the trilinear scalar interaction strengths of eq. (2.17). By default, NLOCT renormalises them in the $\overline{\mathrm{MS}}$ scheme regardless of any finite pieces that are relevant in the OS scheme. A correct treatment therefore requires to replace them by their analytical expression (that involves the fermion masses), prior to the call to NLOCT. This can be achieved straightforwardly with the RemovingInternalCst method of MoGRE that re-expresses a given parameter in terms of the others. Concretely, this method removes a given parameter and the associated renormalisation constant from the model and replaces them by the corresponding analytical expressions. Whilst the replacement rule of the parameter itself is taken from the model file, the one of the renormalisation constant is derived on the fly.

In the context of the MSSM implementation presented in this paper, we have implemented

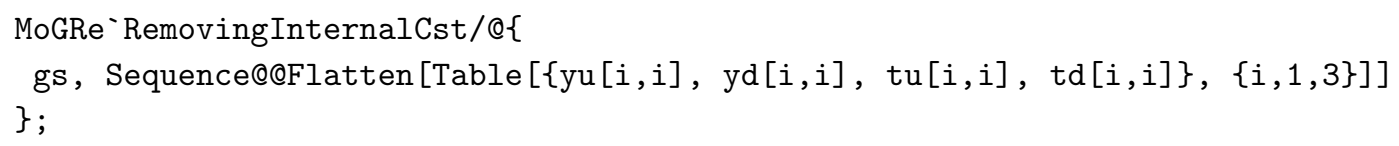

so that all Yukawa and trilinear scalar interactions have been replaced according to their dependence on the external quark masses. We have also made use of this method to define the renormalisation of the strong coupling through $\alpha_{S}$ and not $g_{s}$.

\section{B.6.2 Field renormalisation}

In order to get the list of fields that should be renormalised, the programme starts by extracting from the Lagrangian all relevant interaction terms on the basis of the information passed as the value of the CouplingOrders option of the MoGRe\$Renormalize method (see section B.1). The corresponding field content is subsequently extracted and the relations between the bare and renormalised quantities are derived following eq. (2.21). Matrix renormalisation is by default considered for what concern fields lying under the same representation of the gauge and Poincaré groups. An expansion over all flavour indices is then performed and the field mixing restrictions passed as the FlavorMixing option of the MoGRe\$Renormalize method (see section B.1) are finally enforced.

The wave-function renormalisation constants associated with the left-handed and righthanded chiralities of a Majorana fermion being equal, the code simplifies the resulting 
expression by mapping the right-handed one onto the left-handed one,

$$
\Psi \rightarrow\left[1+\frac{1}{2} \delta Z_{\Psi}\right] \Psi
$$

This has the advantage to prevent NLOCT from calculating twice the same quantity and to yield more compact expressions for the counterterms.

\section{B.6.3 Parameter renormalisation}

The renormalisation of the model parameters is accounted for in three steps. External parameters (including internal parameters that have been made external with FR\$LoopSwitches, see section B.6.1), particle masses and internal parameters (including external parameters that have been made internal with FR\$LoopSwitches) are handled separately. All parameters and masses are directly renormalised according to eq. (2.23), after removing all doubly-declared parameters defined through the FR \$RmDblExt variable (see section B.6.1). The code additionally takes care of deriving all relations connecting the renormalisation constants of the internal parameters to those of other (external or internal) parameters. Those relations are truncated at the one-loop level. For instance, the renormalisation constant associated with the third generation trilinear coupling strength of eq. (2.17), $\delta\left(\hat{\mathbf{T}}^{\mathbf{u}}\right)_{33}$, can be written in terms of the top-quark mass and $\left(\mathbf{A}^{\mathbf{u}}\right)_{33}$ renormalisation constants,

$$
\delta\left(\hat{\mathbf{T}}^{\mathbf{u}}\right)_{33}=\left[\frac{\delta m_{t}}{m_{t}}+\delta\left(\mathbf{A}^{\mathbf{u}}\right)_{33}\right]\left(\hat{\mathbf{T}}^{\mathbf{u}}\right)_{33} .
$$

The obtained set of relations do not include any dependence on the renormalisation constants that would be associated with unrenormalised parameters (see section B.2) and vanishing parameters have been removed (see section B.3).

As a last step, the code applies all renormalisation conditions that have been provided by the user via the AddRenormalizationCondition method. The relations between bare parameters and the corresponding renormalised ones are modified so that the dependent renormalisation constants are replaced by their functional form. This also concerns the rules relating the renormalisation constant of an internal parameter to other parameters and their renormalisation constants.

\section{B.6.4 Renormalisation of the Lagrangian}

All the previously derived parameter and field redefinitions are finally applied to the Lagrangian. A truncation at the one-loop level is performed by the code, so that each Lagrangian term is at most linear in the renormalisation constants. The Lagrangian is then formatted so that NLOCT can be called to derive the UV and $R_{2}$ counterterms, following the techniques detailed in ref. [59]. As a consequence, while MoGRe lifts some of the limitations inherent to a joint use of FEYNRULES and NLOCT, it naturally inherits all limitations that are strictly bounded to NLOCT. For instance, couplings independent of the particle masses are renormalised in the $\overline{\mathrm{MS}}$ scheme and $\alpha_{S}$ has to be renormalised either in the $\overline{\mathrm{MS}}$ scheme or as described in section 2.2.5. This will be addressed in future work. 


\section{Decoupling of heavy SUSY particles in MG5_AMC}

Arbitrary SUSY mass spectra often feature SUSY particles with masses much larger than the collision energy scale, and which are therefore expected to decouple in the corresponding cross-section computations.

In the tree-level matrix elements, this decoupling property applies individually to each tree-level Feynman diagram featuring a propagator of the decoupling heavy SUSY particle. In the loop matrix elements, however, this decoupling is realised in a more complicated way involving cancellations among several loop diagrams featuring the decoupling particle(s) running in the loop. These cancellations become more severe as one approaches the decoupling limit, and numerical inaccuracies in the loop computations will eventually spoil them, yielding incorrect predictions.

The solution to this problem simply amounts to completely removing the problematic heavy modes from the process definition, effectively enforcing the decoupling property of the resulting matrix elements. It is however rather impractical having to settle for different process definitions depending on the masses of the heaviest particles in the spectra, and it is therefore desirable to determine quantitatively when this explicit removal procedure really becomes mandatory from a numerical standpoint. To this end, we consider the one-loop matrix element for the pair production of gluinos from initial-state gluons (i.e. $g g \rightarrow \tilde{g} \tilde{g}$ ), for the reason that this particular process features the worst numerical behaviour in the decoupling limit amongst all $2 \rightarrow 2$ one-loop SUSY matrix elements. We fix the kinematic configuration to $\sqrt{s}=4 m_{\tilde{g}}=2 \mathrm{TeV}$, and report in figure 15 the numerical evaluation of this matrix element as a function of the mass of a decoupling down squark. We observe that the numerical evaluation starts to depart away from the decoupling limit for down-squark masses around $500 \mathrm{TeV}$ in double precision and $5000 \mathrm{TeV}$ in quadruple precision. However, these numerical instabilities will start to significantly impact the accuracy of the final result only when reaching even larger masses of about $10^{3} \mathrm{TeV}$ and $10^{4} \mathrm{TeV}$ respectively. In view of these results, we conclude that a conservative recommendation is to manually enforce the decoupling of certain heavy particles by removing them explicitly from the process definition when their masses are larger than about a thousand times the characteristic energy probed by the observable. We stress however that in any case we tested that the associated numerical instabilities are correctly detected by MADLOoP and will therefore adequately be reported to the integrator, which will in turn set the corresponding weight to zero if the accuracy is too poor. If such exceptional configurations occur too frequently, a clear warning is issued, hence preventing the user from inadvertently using incorrect results. 


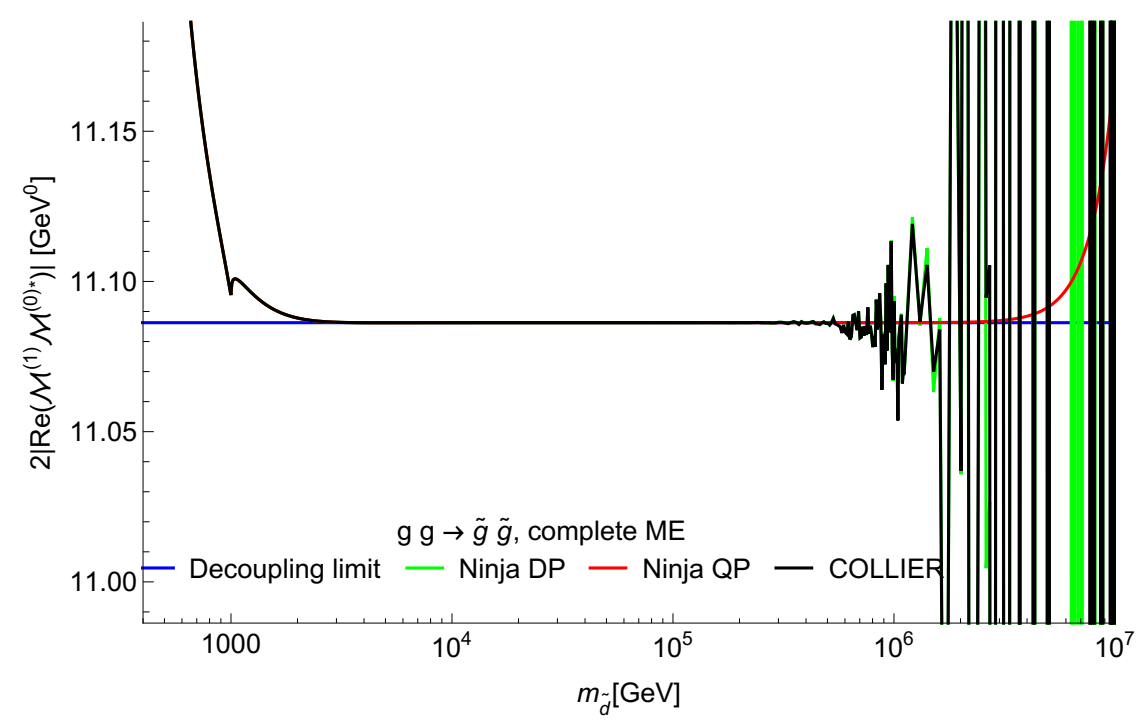

Figure 15. Numerical stability of the one-loop matrix element (ME) associated with the process $g g \rightarrow \tilde{g} \tilde{g}$ as a function of the mass of the down squark $\tilde{d} \equiv \tilde{d}_{L}, \tilde{d}_{R}$. The considered kinematic configuration satisfies $\sqrt{s}=4 m_{\tilde{g}}=2 \mathrm{TeV}$. The constant line in blue indicates the decoupling limit, as obtained from computing the one-loop matrix element in the absence of down squarks. The other lines denote a numerical evaluation using various one-loop reduction algorithms and implementations of scalar master integrals: COLLIER (black) and NiNJA in double (DP, green) and in quadruple (QP, red) precision.

Open Access. This article is distributed under the terms of the Creative Commons Attribution License (CC-BY 4.0), which permits any use, distribution and reproduction in any medium, provided the original author(s) and source are credited.

\section{References}

[1] Yu.A. Golfand and E.P. Likhtman, Extension of the Algebra of Poincaré Group Generators and Violation of p Invariance, JETP Lett. 13 (1971) 323 [Pisma Zh. Eksp. Teor. Fiz. 13 (1971) 452] [INSPIRE].

[2] D.V. Volkov and V.P. Akulov, Is the Neutrino a Goldstone Particle?, Phys. Lett. 46B (1973) 109 [INSPIRE].

[3] J. Wess and B. Zumino, A Lagrangian Model Invariant Under Supergauge Transformations, Phys. Lett. 49B (1974) 52 [INSPIRE].

[4] J. Wess and B. Zumino, Supergauge Transformations in Four-Dimensions, Nucl. Phys. B 70 (1974) 39 [INSPIRE].

[5] J. Wess and B. Zumino, Supergauge Invariant Extension of Quantum Electrodynamics, Nucl. Phys. B 78 (1974) 1 [inSPIRE].

[6] A. Salam and J.A. Strathdee, Supergauge Transformations, Nucl. Phys. B 76 (1974) 477 [INSPIRE].

[7] A. Salam and J.A. Strathdee, On Superfields and Fermi-Bose Symmetry, Phys. Rev. D 11 (1975) 1521 [INSPIRE]. 
[8] S. Ferrara, J. Wess and B. Zumino, Supergauge Multiplets and Superfields, Phys. Lett. 51B (1974) 239 [INSPIRE].

[9] S. Ferrara and B. Zumino, Supergauge Invariant Yang-Mills Theories, Nucl. Phys. B 79 (1974) 413 [INSPIRE].

[10] H.P. Nilles, Supersymmetry, Supergravity and Particle Physics, Phys. Rept. 110 (1984) 1 [INSPIRE].

[11] H.E. Haber and G.L. Kane, The Search for Supersymmetry: Probing Physics Beyond the Standard Model, Phys. Rept. 117 (1985) 75 [inSPIRE].

[12] E. Witten, Dynamical Breaking of Supersymmetry, Nucl. Phys. B 188 (1981) 513 [InSPIRE].

[13] L.E. Ibáñez and G.G. Ross, Low-Energy Predictions in Supersymmetric Grand Unified Theories, Phys. Lett. 105B (1981) 439 [InSPIRE].

[14] S. Dimopoulos, S. Raby and F. Wilczek, Supersymmetry and the Scale of Unification, Phys. Rev. D 24 (1981) 1681 [INSPIRE].

[15] J.R. Ellis, S. Kelley and D.V. Nanopoulos, Probing the desert using gauge coupling unification, Phys. Lett. B 260 (1991) 131 [INSPIRE].

[16] H. Goldberg, Constraint on the Photino Mass from Cosmology, Phys. Rev. Lett. 50 (1983) 1419 [Erratum ibid. 103 (2009) 099905] [INSPIRE].

[17] J.R. Ellis, J.S. Hagelin, D.V. Nanopoulos, K.A. Olive and M. Srednicki, Supersymmetric Relics from the Big Bang, Nucl. Phys. B 238 (1984) 453 [inSPIRE].

[18] ATLAS collaboration, Search for supersymmetry in final states with missing transverse momentum and multiple b-jets in proton-proton collisions at $\sqrt{s}=13 \mathrm{TeV}$ with the ATLAS detector, ATLAS-CONF-2018-041 (2018).

[19] ATLAS collaboration, Search for bottom-squark pair production with the ATLAS detector in final states containing Higgs bosons, b-jets and missing transverse momentum, ATLAS-CONF-2019-011 (2019).

[20] CMS collaboration, Searches for new phenomena in events with jets and high values of the $M_{\mathrm{T} 2}$ variable, including signatures with disappearing tracks, in proton-proton collisions at $\sqrt{s}=13 \mathrm{TeV}$, CMS-PAS-SUS-19-005 (2019).

[21] CMS collaboration, Search for physics beyond the standard model in events with two same-sign leptons or at least three leptons and jets in proton-proton collisions at $\sqrt{s}=13 \mathrm{TeV}$., CMS-PAS-SUS-19-008 (2019).

[22] ATLAS collaboration, Search for electroweak production of charginos and sleptons decaying in final states with two leptons and missing transverse momentum in $\sqrt{s}=13 \mathrm{TeV} \mathrm{pp}$ collisions using the ATLAS detector, ATLAS-CONF-2019-008 (2019).

[23] CMS collaboration, Search for direct $\tau$ slepton pair production in proton-proton collisions at $\sqrt{s}=13 \mathrm{TeV}$, CMS-PAS-SUS-18-006 (2018).

[24] J. Alwall, P. Schuster and N. Toro, Simplified Models for a First Characterization of New Physics at the LHC, Phys. Rev. D 79 (2009) 075020 [arXiv:0810.3921] [inSPIRE].

[25] LHC New Physics Working Group collaboration, Simplified Models for LHC New Physics Searches, J. Phys. G 39 (2012) 105005 [arXiv:1105.2838] [InSPIRE].

[26] S. Catani, F. Krauss, R. Kuhn and B.R. Webber, QCD matrix elements + parton showers, JHEP 11 (2001) 063 [hep-ph/0109231] [INSPIRE].

[27] F. Krauss, Matrix elements and parton showers in hadronic interactions, JHEP 08 (2002) 015 [hep-ph/0205283] [INSPIRE]. 
[28] M.L. Mangano, M. Moretti, F. Piccinini and M. Treccani, Matching matrix elements and shower evolution for top-quark production in hadronic collisions, JHEP 01 (2007) 013 [hep-ph/0611129] [INSPIRE].

[29] L. Lönnblad, Correcting the color dipole cascade model with fixed order matrix elements, JHEP 05 (2002) 046 [hep-ph/0112284] [INSPIRE].

[30] N. Lavesson and L. Lönnblad, W+jets matrix elements and the dipole cascade, JHEP 07 (2005) 054 [hep-ph/0503293] [INSPIRE].

[31] S. Hoeche, F. Krauss, S. Schumann and F. Siegert, QCD matrix elements and truncated showers, JHEP 05 (2009) 053 [arXiv:0903.1219] [INSPIRE].

[32] K. Hamilton, P. Richardson and J. Tully, A modified CKKW matrix element merging approach to angular-ordered parton showers, JHEP 11 (2009) 038 [arXiv:0905.3072] [INSPIRE].

[33] L. Lönnblad and S. Prestel, Matching Tree-Level Matrix Elements with Interleaved Showers, JHEP 03 (2012) 019 [arXiv:1109.4829] [inSPIRE].

[34] S. Frixione and B.R. Webber, Matching NLO QCD computations and parton shower simulations, JHEP 06 (2002) 029 [hep-ph/0204244] [INSPIRE].

[35] P. Nason, A new method for combining NLO QCD with shower Monte Carlo algorithms, JHEP 11 (2004) 040 [hep-ph/0409146] [INSPIRE].

[36] Z. Nagy and D.E. Soper, Matching parton showers to NLO computations, JHEP 10 (2005) 024 [hep-ph/0503053] [inSPIRE].

[37] C.W. Bauer and M.D. Schwartz, Event Generation from Effective Field Theory, Phys. Rev. D 76 (2007) 074004 [hep-ph/0607296] [INSPIRE].

[38] Z. Nagy and D.E. Soper, Parton showers with quantum interference, JHEP 09 (2007) 114 [arXiv:0706.0017] [INSPIRE].

[39] W.T. Giele, D.A. Kosower and P.Z. Skands, A simple shower and matching algorithm, Phys. Rev. D 78 (2008) 014026 [arXiv:0707.3652] [InSPIRE].

[40] C.W. Bauer, F.J. Tackmann and J. Thaler, GenEvA. I. A new framework for event generation, JHEP 12 (2008) 010 [arXiv:0801.4026] [INSPIRE].

[41] S. Hoeche, F. Krauss, M. Schonherr and F. Siegert, A critical appraisal of $N L O+P S$ matching methods, JHEP 09 (2012) 049 [arXiv:1111.1220] [INSPIRE].

[42] K. Hamilton, P. Nason, E. Re and G. Zanderighi, NNLOPS simulation of Higgs boson production, JHEP 10 (2013) 222 [arXiv:1309.0017] [INSPIRE].

[43] S. Jadach, W. Płaczek, S. Sapeta, A. Siódmok and M. Skrzypek, Matching NLO QCD with parton shower in Monte Carlo scheme - the KrkNLO method, JHEP 10 (2015) 052 [arXiv: 1503.06849] [INSPIRE].

[44] N. Lavesson and L. Lönnblad, Extending CKKW-merging to One-Loop Matrix Elements, JHEP 12 (2008) 070 [arXiv:0811.2912] [InSPIRE].

[45] K. Hamilton and P. Nason, Improving NLO-parton shower matched simulations with higher order matrix elements, JHEP 06 (2010) 039 [arXiv:1004.1764] [INSPIRE].

[46] S. Hoche, F. Krauss, M. Schonherr and F. Siegert, NLO matrix elements and truncated showers, JHEP 08 (2011) 123 [arXiv: 1009.1127] [INSPIRE].

[47] W.T. Giele, D.A. Kosower and P.Z. Skands, Higher-Order Corrections to Timelike Jets, Phys. Rev. D 84 (2011) 054003 [arXiv:1102.2126] [InSPIRE]. 
[48] S. Alioli, K. Hamilton and E. Re, Practical improvements and merging of POWHEG simulations for vector boson production, JHEP 09 (2011) 104 [arXiv:1108.0909] [INSPIRE].

[49] S. Hoeche, F. Krauss, M. Schonherr and F. Siegert, QCD matrix elements + parton showers: The NLO case, JHEP 04 (2013) 027 [arXiv: 1207.5030] [INSPIRE].

[50] R. Frederix and S. Frixione, Merging meets matching in MC@NLO, JHEP 12 (2012) 061 [arXiv: 1209.6215] [INSPIRE].

[51] S. Plätzer, Controlling inclusive cross sections in parton shower + matrix element merging, JHEP 08 (2013) 114 [arXiv:1211.5467] [INSPIRE].

[52] S. Alioli et al., Combining Higher-Order Resummation with Multiple NLO Calculations and Parton Showers in GENEVA, JHEP 09 (2013) 120 [arXiv: 1211.7049] [INSPIRE].

[53] L. Lönnblad and S. Prestel, Merging Multi-leg NLO Matrix Elements with Parton Showers, JHEP 03 (2013) 166 [arXiv:1211.7278] [InSPIRE].

[54] K. Hamilton, P. Nason, C. Oleari and G. Zanderighi, Merging $H / W / Z+0$ and 1 jet at NLO with no merging scale: a path to parton shower + NNLO matching, JHEP 05 (2013) 082 [arXiv: 1212.4504] [INSPIRE].

[55] S. Alioli, C.W. Bauer, C. Berggren, F.J. Tackmann, J.R. Walsh and S. Zuberi, Matching Fully Differential NNLO Calculations and Parton Showers, JHEP 06 (2014) 089 [arXiv:1311.0286] [INSPIRE].

[56] J. Alwall et al., The automated computation of tree-level and next-to-leading order differential cross sections and their matching to parton shower simulations, JHEP $\mathbf{0 7}$ (2014) 079 [arXiv: 1405.0301] [INSPIRE].

[57] C. Degrande, C. Duhr, B. Fuks, D. Grellscheid, O. Mattelaer and T. Reiter, UFO - The Universal FeynRules Output, Comput. Phys. Commun. 183 (2012) 1201 [arXiv:1108.2040] [INSPIRE].

[58] A. Alloul, N.D. Christensen, C. Degrande, C. Duhr and B. Fuks, FeynRules 2.0 - A complete toolbox for tree-level phenomenology, Comput. Phys. Commun. 185 (2014) 2250 [arXiv:1310.1921] [INSPIRE].

[59] C. Degrande, Automatic evaluation of UV and R2 terms for beyond the Standard Model Lagrangians: a proof-of-principle, Comput. Phys. Commun. 197 (2015) 239 [arXiv: 1406.3030] [INSPIRE].

[60] G.R. Farrar and P. Fayet, Phenomenology of the Production, Decay and Detection of New Hadronic States Associated with Supersymmetry, Phys. Lett. 76B (1978) 575 [inSPIRE].

[61] J. Wess and J. Bagger, Supersymmetry and supergravity, Princeton University Press, (1992).

[62] B. Fuks, Supersymmetry - When Theory Inspires Experimental Searches, Habilitation thesis, Strasbourg University, 2013, arXiv:1401.6277 [INSPIRE].

[63] L. Girardello and M.T. Grisaru, Soft Breaking of Supersymmetry, Nucl. Phys. B 194 (1982) 65 [INSPIRE].

[64] L.J. Hall, V.A. Kostelecky and S. Raby, New Flavor Violations in Supergravity Models, Nucl. Phys. B 267 (1986) 415 [InSPIRE].

[65] P.Z. Skands et al., SUSY Les Houches accord: Interfacing SUSY spectrum calculators, decay packages and event generators, JHEP 07 (2004) 036 [hep-ph/0311123] [INSPIRE].

[66] P.H. Chankowski, S. Pokorski and J. Rosiek, Complete on-shell renormalization scheme for the minimal supersymmetric Higgs sector, Nucl. Phys. B 423 (1994) 437 [hep-ph/9303309] [INSPIRE]. 
[67] A. Dabelstein, The one loop renormalization of the MSSM Higgs sector and its application to the neutral scalar Higgs masses, Z. Phys. C 67 (1995) 495 [hep-ph/9409375] [InSPIRE].

[68] D.M. Pierce, J.A. Bagger, K.T. Matchev and R.-j. Zhang, Precision corrections in the minimal supersymmetric standard model, Nucl. Phys. B 491 (1997) 3 [hep-ph/9606211] [INSPIRE].

[69] M.D. Goodsell, K. Nickel and F. Staub, Two-Loop Higgs mass calculations in supersymmetric models beyond the MSSM with SARAH and SPheno, Eur. Phys. J. C 75 (2015) 32 [arXiv:1411.0675] [INSPIRE].

[70] R. Frederix, S. Frixione, V. Hirschi, D. Pagani, H.S. Shao and M. Zaro, The automation of next-to-leading order electroweak calculations, JHEP 07 (2018) 185 [arXiv:1804.10017] [INSPIRE].

[71] G. Passarino and M.J.G. Veltman, One Loop Corrections for $e^{+} e^{-}$Annihilation Into $\mu^{+} \mu^{-}$ in the Weinberg Model, Nucl. Phys. B 160 (1979) 151 [INSPIRE].

[72] H. Eberl, A. Bartl and W. Majerotto, SUSY QCD corrections to scalar quark pair production in $e^{+} e^{-}$annihilation, Nucl. Phys. B 472 (1996) 481 [hep-ph/9603206] [INSPIRE].

[73] C. Degrande, B. Fuks, V. Hirschi, J. Proudom and H.-S. Shao, Automated next-to-leading order predictions for new physics at the LHC: the case of colored scalar pair production, Phys. Rev. D 91 (2015) 094005 [arXiv:1412.5589] [INSPIRE].

[74] C. Degrande, B. Fuks, V. Hirschi, J. Proudom and H.-S. Shao, Matching next-to-leading order predictions to parton showers in supersymmetric QCD, Phys. Lett. B $\mathbf{7 5 5}$ (2016) 82 [arXiv: 1510.00391] [INSPIRE].

[75] Y. Yamada, Radiative corrections to sfermion mass splittings, Phys. Rev. D 54 (1996) 1150 [hep-ph/9602279] [INSPIRE].

[76] A. Bartl, H. Eberl, K. Hidaka, T. Kon, W. Majerotto and Y. Yamada, QCD corrections to Higgs boson decays into squarks in the minimal supersymmetric standard model, Phys. Lett. B 402 (1997) 303 [hep-ph/9701398] [INSPIRE].

[77] A. Bartl et al., SUSY - QCD corrections to top and bottom squark decays into Higgs bosons, Phys. Rev. D 59 (1999) 115007 [hep-ph/9806299] [INSPIRE].

[78] H. Eberl, K. Hidaka, S. Kraml, W. Majerotto and Y. Yamada, Improved SUSY QCD corrections to Higgs boson decays into quarks and squarks, Phys. Rev. D 62 (2000) 055006 [hep-ph/9912463] [INSPIRE].

[79] W. Hollik and H. Rzehak, The sfermion mass spectrum of the MSSM at the one loop level, Eur. Phys. J. C 32 (2003) 127 [hep-ph/0305328] [inSPIRE].

[80] S. Heinemeyer, W. Hollik, H. Rzehak and G. Weiglein, High-precision predictions for the MSSM Higgs sector at $O\left(\alpha_{b} \alpha_{s}\right)$, Eur. Phys. J. C 39 (2005) 465 [hep-ph/0411114] [INSPIRE].

[81] W. Hollik and E. Mirabella, Squark anti-squark pair production at the LHC: The electroweak contribution, JHEP 12 (2008) 087 [arXiv:0806.1433] [INSPIRE].

[82] A. Brignole, G. Degrassi, P. Slavich and F. Zwirner, On the two loop sbottom corrections to the neutral Higgs boson masses in the MSSM, Nucl. Phys. B 643 (2002) 79 [hep-ph/0206101] [INSPIRE].

[83] G. Degrassi and P. Slavich, NLO QCD bottom corrections to Higgs boson production in the MSSM, JHEP 11 (2010) 044 [arXiv: 1007.3465] [INSPIRE]. 
[84] S. Heinemeyer, H. Rzehak and C. Schappacher, Proposals for Bottom Quark/Squark Renormalization in the Complex MSSM, Phys. Rev. D 82 (2010) 075010 [arXiv: 1007.0689] [INSPIRE].

[85] J.C. Collins, F. Wilczek and A. Zee, Low-Energy Manifestations of Heavy Particles: Application to the Neutral Current, Phys. Rev. D 18 (1978) 242 [InSPIRE].

[86] W.A. Bardeen, A.J. Buras, D.W. Duke and T. Muta, Deep Inelastic Scattering Beyond the Leading Order in Asymptotically Free Gauge Theories, Phys. Rev. D 18 (1978) 3998 [INSPIRE].

[87] W.J. Marciano, Flavor Thresholds and $\Lambda$ in the Modified Minimal Subtraction Prescription, Phys. Rev. D 29 (1984) 580 [inSPIRE].

[88] S.P. Martin and M.T. Vaughn, Regularization dependence of running couplings in softly broken supersymmetry, Phys. Lett. B 318 (1993) 331 [hep-ph/9308222] [INSPIRE].

[89] T. Hahn, Generating Feynman diagrams and amplitudes with FeynArts 3, Comput. Phys. Commun. 140 (2001) 418 [hep-ph/0012260] [INSPIRE].

[90] B. Fuks, K. Nordström, R. Ruiz and S.L. Williamson, Sleptons without Hadrons, Phys. Rev. D 100 (2019) 074010 [arXiv:1901.09937] [InSPIRE].

[91] C. Duhr and B. Fuks, A superspace module for the FeynRules package, Comput. Phys. Commun. 182 (2011) 2404 [arXiv:1102.4191] [INSPIRE].

[92] W. Beenakker, R. Hopker, M. Spira and P.M. Zerwas, Squark production at the Tevatron, Phys. Rev. Lett. 74 (1995) 2905 [hep-ph/9412272] [INSPIRE].

[93] W. Beenakker, R. Hopker, M. Spira and P.M. Zerwas, Squark and gluino production at hadron colliders, Nucl. Phys. B 492 (1997) 51 [hep-ph/9610490] [INSPIRE].

[94] G. Bozzi, B. Fuks and M. Klasen, Non-diagonal and mixed squark production at hadron colliders, Phys. Rev. D 72 (2005) 035016 [hep-ph/0507073] [INSPIRE].

[95] A. Kulesza and L. Motyka, Threshold resummation for squark-antisquark and gluino-pair production at the LHC, Phys. Rev. Lett. 102 (2009) 111802 [arXiv:0807.2405] [INSPIRE].

[96] A. Kulesza and L. Motyka, Soft gluon resummation for the production of gluino-gluino and squark-antisquark pairs at the LHC, Phys. Rev. D 80 (2009) 095004 [arXiv:0905.4749] [INSPIRE].

[97] W. Beenakker, S. Brensing, M. Krämer, A. Kulesza, E. Laenen and I. Niessen, Soft-gluon resummation for squark and gluino hadroproduction, JHEP 12 (2009) 041 [arXiv:0909.4418] [INSPIRE].

[98] W. Beenakker et al., Squark and Gluino Hadroproduction, Int. J. Mod. Phys. A 26 (2011) 2637 [arXiv: 1105.1110] [INSPIRE].

[99] M.R. Kauth, J.H. Kuhn, P. Marquard and M. Steinhauser, Gluino Pair Production at the LHC: The Threshold, Nucl. Phys. B 857 (2012) 28 [arXiv:1108.0361] [inSPIRE].

[100] P. Falgari, C. Schwinn and C. Wever, NLL soft and Coulomb resummation for squark and gluino production at the LHC, JHEP 06 (2012) 052 [arXiv: 1202.2260] [INSPIRE].

[101] W. Beenakker, M. Krämer, T. Plehn, M. Spira and P.M. Zerwas, Stop production at hadron colliders, Nucl. Phys. B 515 (1998) 3 [hep-ph/9710451] [InSPIRE].

[102] W. Beenakker, S. Brensing, M. Krämer, A. Kulesza, E. Laenen and I. Niessen, Supersymmetric top and bottom squark production at hadron colliders, JHEP 08 (2010) 098 [arXiv: 1006.4771] [INSPIRE]. 
[103] M. Beneke, P. Falgari and C. Schwinn, Threshold resummation for pair production of coloured heavy (s)particles at hadron colliders, Nucl. Phys. B 842 (2011) 414 [arXiv: 1007.5414] [INSPIRE].

[104] W. Beenakker, R. Hopker, M. Spira and P.M. Zerwas, Gluino pair production at the Tevatron, Z. Phys. C 69 (1995) 163 [hep-ph/9505416] [INSPIRE].

[105] W. Beenakker, M. Klasen, M. Krämer, T. Plehn, M. Spira and P.M. Zerwas, The production of charginos/neutralinos and sleptons at hadron colliders, Phys. Rev. Lett. 83 (1999) 3780 [Erratum ibid. 100 (2008) 029901] [hep-ph/9906298] [INSPIRE].

[106] J. Debove, B. Fuks and M. Klasen, Transverse-momentum resummation for gaugino-pair production at hadron colliders, Phys. Lett. B 688 (2010) 208 [arXiv:0907.1105] [INSPIRE].

[107] J. Debove, B. Fuks and M. Klasen, Threshold resummation for gaugino pair production at hadron colliders, Nucl. Phys. B 842 (2011) 51 [arXiv:1005.2909] [INSPIRE].

[108] J. Debove, B. Fuks and M. Klasen, Joint Resummation for Gaugino Pair Production at Hadron Colliders, Nucl. Phys. B 849 (2011) 64 [arXiv:1102.4422] [INSPIRE].

[109] B. Fuks, M. Klasen, D.R. Lamprea and M. Rothering, Gaugino production in proton-proton collisions at a center-of-mass energy of 8 TeV, JHEP 10 (2012) 081 [arXiv:1207.2159] [INSPIRE].

[110] G. Bozzi, B. Fuks and M. Klasen, Transverse-momentum resummation for slepton-pair production at the CERN LHC, Phys. Rev. D 74 (2006) 015001 [hep-ph/0603074] [INSPIRE].

[111] G. Bozzi, B. Fuks and M. Klasen, Threshold Resummation for Slepton-Pair Production at Hadron Colliders, Nucl. Phys. B 777 (2007) 157 [hep-ph/0701202] [INSPIRE].

[112] G. Bozzi, B. Fuks and M. Klasen, Joint resummation for slepton pair production at hadron colliders, Nucl. Phys. B 794 (2008) 46 [arXiv:0709.3057] [INSPIRE].

[113] B. Fuks, M. Klasen, D.R. Lamprea and M. Rothering, Revisiting slepton pair production at the Large Hadron Collider, JHEP 01 (2014) 168 [arXiv:1310.2621] [INSPIRE].

[114] E.L. Berger, M. Klasen and T.M.P. Tait, Next-to-leading order SUSY QCD predictions for associated production of gauginos and gluinos, Phys. Rev. D 62 (2000) 095014 [hep-ph/0212306] [INSPIRE].

[115] B. Fuks, M. Klasen and M. Rothering, Soft gluon resummation for associated gluino-gaugino production at the LHC, JHEP 07 (2016) 053 [arXiv: 1604.01023] [INSPIRE].

[116] W. Hollik, J.M. Lindert and D. Pagani, NLO corrections to squark-squark production and decay at the LHC, JHEP 03 (2013) 139 [arXiv:1207.1071] [INSPIRE].

[117] W. Hollik, J.M. Lindert and D. Pagani, On cascade decays of squarks at the LHC in NLO QCD, Eur. Phys. J. C 73 (2013) 2410 [arXiv:1303.0186] [INSPIRE].

[118] U. Langenfeld and S.-O. Moch, Higher-order soft corrections to squark hadro-production, Phys. Lett. B 675 (2009) 210 [arXiv:0901.0802] [INSPIRE].

[119] U. Langenfeld, Threshold Improved QCD Corrections for Stop-Antistop production at Hadron colliders, JHEP 07 (2011) 052 [arXiv:1011.3341] [INSPIRE].

[120] U. Langenfeld, S.-O. Moch and T. Pfoh, QCD threshold corrections for gluino pair production at hadron colliders, JHEP 11 (2012) 070 [arXiv:1208.4281] [INSPIRE].

[121] W. Hollik, M. Kollar and M.K. Trenkel, Hadronic production of top-squark pairs with electroweak NLO contributions, JHEP 02 (2008) 018 [arXiv: 0712.0287] [INSPIRE]. 
[122] M. Beccaria, G. Macorini, L. Panizzi, F.M. Renard and C. Verzegnassi, Stop-antistop and sbottom-antisbottom production at LHC: A One-loop search for model parameters dependence, Int. J. Mod. Phys. A 23 (2008) 4779 [arXiv:0804.1252] [InSPIRE].

[123] W. Hollik, E. Mirabella and M.K. Trenkel, Electroweak contributions to squark-gluino production at the LHC, JHEP 02 (2009) 002 [arXiv:0810.1044] [INSPIRE].

[124] E. Mirabella, NLO electroweak contributions to gluino pair production at hadron colliders, JHEP 12 (2009) 012 [arXiv:0908.3318] [INSPIRE].

[125] A. Arhrib, R. Benbrik, K. Cheung and T.-C. Yuan, Higgs boson enhancement effects on squark-pair production at the LHC, JHEP 02 (2010) 048 [arXiv:0911.1820] [INSPIRE].

[126] J. Germer, W. Hollik, E. Mirabella and M.K. Trenkel, Hadronic production of squark-squark pairs: The electroweak contributions, JHEP 08 (2010) 023 [arXiv: 1004.2621] [INSPIRE].

[127] J. Germer, W. Hollik and E. Mirabella, Hadronic production of bottom-squark pairs with electroweak contributions, JHEP 05 (2011) 068 [arXiv:1103.1258] [INSPIRE].

[128] J. Germer, W. Hollik, J.M. Lindert and E. Mirabella, Top-squark pair production at the LHC: a complete analysis at next-to-leading order, JHEP 09 (2014) 022 [arXiv:1404.5572] [INSPIRE].

[129] W. Hollik, J.M. Lindert, E. Mirabella and D. Pagani, Electroweak corrections to squark-antisquark production at the LHC, JHEP 08 (2015) 099 [arXiv:1506.01052] [INSPIRE].

[130] W. Beenakker, S. Brensing, M. Krämer, A. Kulesza, E. Laenen and I. Niessen, NNLL resummation for squark-antisquark pair production at the LHC, JHEP 01 (2012) 076 [arXiv:1110.2446] [INSPIRE].

[131] T. Pfoh, Phenomenology of QCD threshold resummation for gluino pair production at NNLL, JHEP 05 (2013) 044 [Erratum ibid. 10 (2013) 090] [arXiv: 1302.7202] [INSPIRE].

[132] A. Broggio, A. Ferroglia, M. Neubert, L. Vernazza and L.L. Yang, Approximate NNLO Predictions for the Stop-Pair Production Cross Section at the LHC, JHEP 07 (2013) 042 [arXiv:1304.2411] [INSPIRE].

[133] W. Beenakker et al., Towards NNLL resummation: hard matching coefficients for squark and gluino hadroproduction, JHEP 10 (2013) 120 [arXiv:1304.6354] [INSPIRE].

[134] W. Beenakker et al., NNLL resummation for squark and gluino production at the LHC, JHEP 12 (2014) 023 [arXiv: 1404.3134] [InSPIRE].

[135] W. Beenakker, C. Borschensky, R. Heger, M. Krämer, A. Kulesza and E. Laenen, NNLL resummation for stop pair-production at the LHC, JHEP 05 (2016) 153 [arXiv: 1601.02954] [INSPIRE].

[136] M. Beneke, J. Piclum, C. Schwinn and C. Wever, NNLL soft and Coulomb resummation for squark and gluino production at the LHC, JHEP 10 (2016) 054 [arXiv:1607.07574] [INSPIRE].

[137] A. Alves, O. Eboli and T. Plehn, Stop lepton associated production at hadron colliders, Phys. Lett. B 558 (2003) 165 [hep-ph/0211441] [INSPIRE].

[138] X.-P. Li, L. Guo, W.-G. Ma, L. Han, R.-Y. Zhang and S.-M. Wang, Single Slepton Production Associated with a Top Quark at LHC in NLO QCD, Eur. Phys. J. C 72 (2012) 1918 [arXiv: 1202.5592] [INSPIRE].

[139] G. Bozzi, B. Fuks, B. Herrmann and M. Klasen, Squark and gaugino hadroproduction and decays in non-minimal flavour violating supersymmetry, Nucl. Phys. B 787 (2007) 1 [arXiv:0704.1826] [INSPIRE]. 
[140] B. Fuks, B. Herrmann and M. Klasen, Flavour Violation in Gauge-Mediated Supersymmetry Breaking Models: Experimental Constraints and Phenomenology at the LHC, Nucl. Phys. B 810 (2009) 266 [arXiv:0808.1104] [InSPIRE].

[141] B. Fuks, B. Herrmann and M. Klasen, Phenomenology of anomaly-mediated supersymmetry breaking scenarios with non-minimal flavour violation, Phys. Rev. D 86 (2012) 015002 [arXiv:1112.4838] [INSPIRE].

[142] T. Binoth, D. Gonçalves Netto, D. Lopez-Val, K. Mawatari, T. Plehn and I. Wigmore, Automized Squark-Neutralino Production to Next-to-Leading Order, Phys. Rev. D 84 (2011) 075005 [arXiv: 1108.1250] [INSPIRE].

[143] D. Gonçalves-Netto, D. López-Val, K. Mawatari, T. Plehn and I. Wigmore, Automated Squark and Gluino Production to Next-to-Leading Order, Phys. Rev. D 87 (2013) 014002 [arXiv: 1211.0286] [INSPIRE].

[144] D. Gonçalves, D. Lopez-Val, K. Mawatari and T. Plehn, Automated third generation squark production to next-to-leading order, Phys. Rev. D 90 (2014) 075007 [arXiv:1407.4302] [INSPIRE].

[145] W. Beenakker et al., NLO+NLL squark and gluino production cross-sections with threshold-improved parton distributions, Eur. Phys. J. C 76 (2016) 53 [arXiv:1510.00375] [INSPIRE].

[146] W. Beenakker, C. Borschensky, M. Krämer, A. Kulesza and E. Laenen, NNLL-fast: predictions for coloured supersymmetric particle production at the LHC with threshold and Coulomb resummation, JHEP 12 (2016) 133 [arXiv:1607.07741] [INSPIRE].

[147] B. Fuks, M. Klasen, D.R. Lamprea and M. Rothering, Precision predictions for electroweak superpartner production at hadron colliders with Resummino, Eur. Phys. J. C 73 (2013) 2480 [arXiv: 1304.0790] [INSPIRE].

[148] R. Gavin et al., Matching Squark Pair Production at NLO with Parton Showers, JHEP 10 (2013) 187 [arXiv: 1305.4061] [InSPIRE].

[149] R. Gavin et al., Squark Production and Decay matched with Parton Showers at NLO, Eur. Phys. J. C 75 (2015) 29 [arXiv:1407.7971] [INSPIRE].

[150] J. Baglio, B. Jäger and M. Kesenheimer, Electroweakino pair production at the LHC: NLO SUSY-QCD corrections and parton-shower effects, JHEP 07 (2016) 083

[arXiv: 1605. 06509] [INSPIRE].

[151] J. Baglio, B. Jäger and M. Kesenheimer, Precise predictions for electroweakino-pair production in association with a jet at the LHC, JHEP 07 (2018) 055 [arXiv:1711.00730] [INSPIRE].

[152] B. Jäger, A. von Manteuffel and S. Thier, Slepton pair production in the POWHEG BOX, JHEP 10 (2012) 130 [arXiv:1208.2953] [inSPIRE].

[153] B. Jäger, A. von Manteuffel and S. Thier, Slepton pair production in association with a jet: NLO-QCD corrections and parton-shower effects, JHEP 02 (2015) 041 [arXiv:1410.3802] [INSPIRE].

[154] S. Frixione, Z. Kunszt and A. Signer, Three jet cross-sections to next-to-leading order, Nucl. Phys. B 467 (1996) 399 [hep-ph/9512328] [INSPIRE].

[155] S. Frixione, A general approach to jet cross-sections in QCD, Nucl. Phys. B 507 (1997) 295 [hep-ph/9706545] [INSPIRE]. 
[156] R. Frederix, S. Frixione, F. Maltoni and T. Stelzer, Automation of next-to-leading order computations in QCD: The FKS subtraction, JHEP 10 (2009) 003 [arXiv:0908.4272] [INSPIRE].

[157] R. Frederix, S. Frixione, A.S. Papanastasiou, S. Prestel and P. Torrielli, Off-shell single-top production at NLO matched to parton showers, JHEP 06 (2016) 027 [arXiv: 1603.01178] [INSPIRE].

[158] G. Ossola, C.G. Papadopoulos and R. Pittau, Reducing full one-loop amplitudes to scalar integrals at the integrand level, Nucl. Phys. B 763 (2007) 147 [hep-ph/0609007] [INSPIRE].

[159] P. Mastrolia, E. Mirabella and T. Peraro, Integrand reduction of one-loop scattering amplitudes through Laurent series expansion, JHEP 06 (2012) 095 [Erratum ibid. 11 (2012) 128] [arXiv: 1203.0291] [INSPIRE].

[160] A.I. Davydychev, A simple formula for reducing Feynman diagrams to scalar integrals, Phys. Lett. B 263 (1991) 107 [INSPIRE].

[161] A. Denner and S. Dittmaier, Reduction schemes for one-loop tensor integrals, Nucl. Phys. B 734 (2006) 62 [hep-ph/0509141] [INSPIRE].

[162] V. Hirschi, R. Frederix, S. Frixione, M.V. Garzelli, F. Maltoni and R. Pittau, Automation of one-loop QCD corrections, JHEP 05 (2011) 044 [arXiv:1103.0621] [INSPIRE].

[163] G. Ossola, C.G. Papadopoulos and R. Pittau, CutTools: A program implementing the OPP reduction method to compute one-loop amplitudes, JHEP 03 (2008) 042 [arXiv:0711.3596] [INSPIRE].

[164] T. Peraro, Ninja: Automated Integrand Reduction via Laurent Expansion for One-Loop Amplitudes, Comput. Phys. Commun. 185 (2014) 2771 [arXiv:1403.1229] [INSPIRE].

[165] V. Hirschi and T. Peraro, Tensor integrand reduction via Laurent expansion, JHEP 06 (2016) 060 [arXiv: 1604.01363] [INSPIRE].

[166] H.-S. Shao, Iregi user manual, unpublished.

[167] A. Denner, S. Dittmaier and L. Hofer, Collier: a fortran-based Complex One-Loop LIbrary in Extended Regularizations, Comput. Phys. Commun. 212 (2017) 220 [arXiv:1604.06792] [INSPIRE].

[168] F. Cascioli, P. Maierhofer and S. Pozzorini, Scattering Amplitudes with Open Loops, Phys. Rev. Lett. 108 (2012) 111601 [arXiv:1111.5206] [InSPIRE].

[169] F. Staub, SARAH 3.2: Dirac Gauginos, UFO output and more, Comput. Phys. Commun. 184 (2013) 1792 [arXiv: 1207.0906] [InSPIRE].

[170] G. Cacciapaglia, E. Conte, A. Deandrea, B. Fuks and H.-S. Shao, LHC constraints and potential on resonant monotop production, Eur. Phys. J. C 79 (2019) 174 [arXiv: 1811.03626] [INSPIRE].

[171] B. Fuks and H.-S. Shao, QCD next-to-leading-order predictions matched to parton showers for vector-like quark models, Eur. Phys. J. C 77 (2017) 135 [arXiv:1610.04622] [InSPIRE].

[172] G. Cacciapaglia et al., Next-to-leading-order predictions for single vector-like quark production at the LHC, Phys. Lett. B 793 (2019) 206 [arXiv:1811.05055] [INSPIRE].

[173] D. Buarque Franzosi, E. Vryonidou and C. Zhang, Scalar production and decay to top quarks including interference effects at NLO in QCD in an EFT approach, JHEP 10 (2017) 096 [arXiv: 1707.06760] [INSPIRE].

[174] G. Das, C. Degrande, V. Hirschi, F. Maltoni and H.-S. Shao, NLO predictions for the production of a spin-two particle at the LHC, Phys. Lett. B 770 (2017) 507 [arXiv: 1605.09359] [INSPIRE]. 
[175] R. Frederix et al., Diphoton production in the ADD model to NLO+parton shower accuracy at the LHC, JHEP 12 (2012) 102 [arXiv: 1209.6527] [INSPIRE].

[176] R. Frederix, M.K. Mandal, P. Mathews, V. Ravindran and S. Seth, Drell-Yan, $Z Z, W^{+} W^{-}$ production in $S M \& A D D$ model to $N L O+P S$ accuracy at the LHC, Eur. Phys. J. C 74 (2014) 2745 [arXiv: 1307.7013] [INSPIRE].

[177] G. Das, P. Mathews, V. Ravindran and S. Seth, RS resonance in di-final state production at the LHC to NLO+PS accuracy, JHEP 10 (2014) 188 [arXiv:1408.3970] [INSPIRE].

[178] C. Degrande, M. Ubiali, M. Wiesemann and M. Zaro, Heavy charged Higgs boson production at the LHC, JHEP 10 (2015) 145 [arXiv: 1507.02549] [INSPIRE].

[179] C. Degrande, R. Frederix, V. Hirschi, M. Ubiali, M. Wiesemann and M. Zaro, Accurate predictions for charged Higgs production: Closing the $m_{H^{ \pm}} \sim m_{t}$ window, Phys. Lett. B 772 (2017) 87 [arXiv:1607.05291] [INSPIRE].

[180] C. Degrande, K. Hartling, H.E. Logan, A.D. Peterson and M. Zaro, Automatic predictions in the Georgi-Machacek model at next-to-leading order accuracy, Phys. Rev. D 93 (2016) 035004 [arXiv: 1512.01243] [INSPIRE].

[181] P. Artoisenet et al., A framework for Higgs characterisation, JHEP 11 (2013) 043 [arXiv:1306.6464] [INSPIRE].

[182] F. Maltoni, K. Mawatari and M. Zaro, Higgs characterisation via vector-boson fusion and associated production: NLO and parton-shower effects, Eur. Phys. J. C 74 (2014) 2710 [arXiv:1311.1829] [INSPIRE].

[183] F. Demartin, F. Maltoni, K. Mawatari, B. Page and M. Zaro, Higgs characterisation at NLO in QCD: CP properties of the top-quark Yukawa interaction, Eur. Phys. J. C 74 (2014) 3065 [arXiv: 1407.5089] [INSPIRE].

[184] F. Demartin, F. Maltoni, K. Mawatari and M. Zaro, Higgs production in association with a single top quark at the LHC, Eur. Phys. J. C 75 (2015) 267 [arXiv:1504.00611] [inSPIRE].

[185] F. Demartin, B. Maier, F. Maltoni, K. Mawatari and M. Zaro, tWH associated production at the LHC, Eur. Phys. J. C 77 (2017) 34 [arXiv: 1607.05862] [InSPIRE].

[186] C. Degrande, B. Fuks, K. Mawatari, K. Mimasu and V. Sanz, Electroweak Higgs boson production in the standard model effective field theory beyond leading order in QCD, Eur. Phys. J. C 77 (2017) 262 [arXiv:1609.04833] [INSPIRE].

[187] C. Degrande, F. Maltoni, J. Wang and C. Zhang, Automatic computations at next-to-leading order in QCD for top-quark flavor-changing neutral processes, Phys. Rev. D 91 (2015) 034024 [arXiv: 1412.5594] [INSPIRE].

[188] G. Durieux, F. Maltoni and C. Zhang, Global approach to top-quark flavor-changing interactions, Phys. Rev. D 91 (2015) 074017 [arXiv:1412.7166] [INSPIRE].

[189] O. Bessidskaia Bylund, F. Maltoni, I. Tsinikos, E. Vryonidou and C. Zhang, Probing top quark neutral couplings in the Standard Model Effective Field Theory at NLO in QCD, JHEP 05 (2016) 052 [arXiv: 1601.08193] [INSPIRE].

[190] F. Maltoni, E. Vryonidou and C. Zhang, Higgs production in association with a top-antitop pair in the Standard Model Effective Field Theory at NLO in QCD, JHEP 10 (2016) 123 [arXiv: 1607.05330] [INSPIRE].

[191] C. Zhang, Single Top Production at Next-to-Leading Order in the Standard Model Effective Field Theory, Phys. Rev. Lett. 116 (2016) 162002 [arXiv:1601.06163] [INSPIRE]. 
[192] D. Buarque Franzosi and C. Zhang, Probing the top-quark chromomagnetic dipole moment at next-to-leading order in QCD, Phys. Rev. D 91 (2015) 114010 [arXiv:1503.08841] [INSPIRE].

[193] V. Hirschi, F. Maltoni, I. Tsinikos and E. Vryonidou, Constraining anomalous gluon self-interactions at the LHC: a reappraisal, JHEP 07 (2018) 093 [arXiv:1806.04696] [INSPIRE].

[194] C. Degrande, A basis of dimension-eight operators for anomalous neutral triple gauge boson interactions, JHEP 02 (2014) 101 [arXiv: 1308.6323] [INSPIRE].

[195] C. Degrande, O. Mattelaer, R. Ruiz and J. Turner, Fully-Automated Precision Predictions for Heavy Neutrino Production Mechanisms at Hadron Colliders, Phys. Rev. D 94 (2016) 053002 [arXiv: 1602.06957] [INSPIRE].

[196] O. Mattelaer, M. Mitra and R. Ruiz, Automated Neutrino Jet and Top Jet Predictions at Next-to-Leading-Order with Parton Shower Matching in Effective Left-Right Symmetric Models, arXiv:1610.08985 [INSPIRE].

[197] B. Fuks and R. Ruiz, A comprehensive framework for studying $W^{\prime}$ and $Z^{\prime}$ bosons at hadron colliders with automated jet veto resummation, JHEP 05 (2017) 032 [arXiv: 1701.05263] [INSPIRE].

[198] O. Mattelaer and E. Vryonidou, Dark matter production through loop-induced processes at the LHC: the s-channel mediator case, Eur. Phys. J. C 75 (2015) 436 [arXiv:1508.00564] [INSPIRE].

[199] M. Backović, M. Krämer, F. Maltoni, A. Martini, K. Mawatari and M. Pellen, Higher-order QCD predictions for dark matter production at the LHC in simplified models with s-channel mediators, Eur. Phys. J. C 75 (2015) 482 [arXiv:1508.05327] [INSPIRE].

[200] M. Neubert, J. Wang and C. Zhang, Higher-Order QCD Predictions for Dark Matter Production in Mono-Z Searches at the LHC, JHEP 02 (2016) 082 [arXiv:1509.05785] [INSPIRE].

[201] C. Arina et al., A comprehensive approach to dark matter studies: exploration of simplified top-philic models, JHEP 11 (2016) 111 [arXiv:1605.09242] [INSPIRE].

[202] S. Kraml, U. Laa, K. Mawatari and K. Yamashita, Simplified dark matter models with a spin-2 mediator at the LHC, Eur. Phys. J. C 77 (2017) 326 [arXiv:1701.07008] [InSPIRE].

[203] N.F. Bell, G. Busoni and I.W. Sanderson, Self-consistent Dark Matter Simplified Models with an s-channel scalar mediator, JCAP 03 (2017) 015 [arXiv:1612.03475] [INSPIRE].

[204] N.F. Bell, G. Busoni and I.W. Sanderson, Two Higgs Doublet Dark Matter Portal, JCAP 01 (2018) 015 [arXiv: 1710.10764] [INSPIRE].

[205] Y. Afik et al., DM+bb simulations with DMSimp: an update, in Dark Matter at the LHC 2018: Experimental and theoretical workshop (DM@LHC 2018) Heidelberg, Germany, April 3-6, 2018, 2018, arXiv:1811.08002 [INSPIRE].

[206] H.-S. Shao and Y.-J. Zhang, Feynman Rules for the Rational Part of One-loop QCD Corrections in the MSSM, JHEP 06 (2012) 112 [arXiv:1205.1273] [INSPIRE].

[207] T. Fritzsche, T. Hahn, S. Heinemeyer, F. von der Pahlen, H. Rzehak and C. Schappacher, The Implementation of the Renormalized Complex MSSM in FeynArts and FormCalc, Comput. Phys. Commun. 185 (2014) 1529 [arXiv:1309.1692] [INSPIRE].

[208] T. Hahn, S. Heinemeyer, F. von der Pahlen, H. Rzehak and C. Schappacher, Renormalization of the Complex MSSM in FeynArts/FormCalc, Nucl. Part. Phys. Proc. 267-269 (2015) 158 [INSPIRE]. 
[209] J. Pumplin, D.R. Stump, J. Huston, H.L. Lai, P.M. Nadolsky and W.K. Tung, New generation of parton distributions with uncertainties from global QCD analysis, JHEP 07 (2002) 012 [hep-ph/0201195] [INSPIRE].

[210] G. Passarino, Peaks and cusps: anomalous thresholds and LHC physics, arXiv:1807.00503 [INSPIRE].

[211] NNPDF collaboration, Parton distributions for the LHC Run II, JHEP 04 (2015) 040 [arXiv: 1410.8849] [INSPIRE].

[212] A. Buckley et al., LHAPDF6: parton density access in the LHC precision era, Eur. Phys. J. C 75 (2015) 132 [arXiv:1412.7420] [InSPIRE].

[213] S. Dulat et al., New parton distribution functions from a global analysis of quantum chromodynamics, Phys. Rev. D 93 (2016) 033006 [arXiv: 1506.07443] [InSPIRE].

[214] ATLAS collaboration, Measurement of the cross-section for producing a $W$ boson in association with a single top quark in pp collisions at $\sqrt{s}=13$ TeV with ATLAS, JHEP 01 (2018) 063 [arXiv: 1612.07231] [InSPIRE].

[215] CMS collaboration, Observation of the associated production of a single top quark and a $W$ boson in pp collisions at $\sqrt{s}=8 \mathrm{TeV}$, Phys. Rev. Lett. 112 (2014) 231802 [arXiv: 1401.2942] [INSPIRE].

[216] S. Frixione, E. Laenen, P. Motylinski, B.R. Webber and C.D. White, Single-top hadroproduction in association with a W boson, JHEP 07 (2008) 029 [arXiv:0805.3067] [INSPIRE].

[217] S. Frixione, E. Laenen, P. Motylinski and B.R. Webber, Angular correlations of lepton pairs from vector boson and top quark decays in Monte Carlo simulations, JHEP 04 (2007) 081 [hep-ph/0702198] [INSPIRE].

[218] A.S. Belyaev, E.E. Boos and L.V. Dudko, Single top quark at future hadron colliders: Complete signal and background study, Phys. Rev. D 59 (1999) 075001 [hep-ph/9806332] [INSPIRE].

[219] T.M.P. Tait, The $t W^{-}$mode of single top production, Phys. Rev. D 61 (1999) 034001 [hep-ph/9909352] [INSPIRE].

[220] S. Zhu, Next-to-leading order QCD corrections to $b g \rightarrow t W^{-}$at CERN large hadron collider, Phys. Lett. B 524 (2002) 283 [Erratum ibid. B 537 (2002) 351] [hep-ph/0109269] [INSPIRE].

[221] E.L. Berger, T. Han, J. Jiang and T. Plehn, Associated production of a top quark and a charged Higgs boson, Phys. Rev. D 71 (2005) 115012 [hep-ph/0312286] [INSPIRE].

[222] J.M. Campbell and F. Tramontano, Next-to-leading order corrections to Wt production and decay, Nucl. Phys. B 726 (2005) 109 [hep-ph/0506289] [INSPIRE].

[223] T.N. Dao, W. Hollik and D.N. Le, $W^{\mp} H^{ \pm}$production and CP asymmetry at the LHC, Phys. Rev. D 83 (2011) 075003 [arXiv:1011.4820] [INSPIRE].

[224] C.D. White, S. Frixione, E. Laenen and F. Maltoni, Isolating Wt production at the LHC, JHEP 11 (2009) 074 [arXiv:0908.0631] [INSPIRE].

[225] C. Weydert et al., Charged Higgs boson production in association with a top quark in MC@NLO, Eur. Phys. J. C 67 (2010) 617 [arXiv:0912.3430] [inSPIRE].

[226] E. Re, Single-top Wt-channel production matched with parton showers using the POWHEG method, Eur. Phys. J. C 71 (2011) 1547 [arXiv: 1009.2450] [INSPIRE]. 
[227] J. Alwall, C. Duhr, B. Fuks, O. Mattelaer, D.G. Öztürk and C.-H. Shen, Computing decay rates for new physics theories with FeynRules and MadGraph 5_aMC@NLO, Comput. Phys. Commun. 197 (2015) 312 [arXiv:1402.1178] [INSPIRE].

[228] O. Mattelaer, On the maximal use of Monte Carlo samples: re-weighting events at NLO accuracy, Eur. Phys. J. C 76 (2016) 674 [arXiv:1607.00763] [INSPIRE].

[229] S. Frixione, P. Nason and C. Oleari, Matching NLO QCD computations with Parton Shower simulations: the POWHEG method, JHEP 11 (2007) 070 [arXiv: 0709.2092] [INSPIRE].

[230] G. Corcella et al., HERWIG 6: An event generator for hadron emission reactions with interfering gluons (including supersymmetric processes), JHEP 01 (2001) 010 [hep-ph/0011363] [INSPIRE].

[231] M. Fairbairn, A.C. Kraan, D.A. Milstead, T. Sjöstrand, P.Z. Skands and T. Sloan, Stable Massive Particles at Colliders, Phys. Rept. 438 (2007) 1 [hep-ph/0611040] [InSPIRE].

[232] N. Desai and P.Z. Skands, Supersymmetry and Generic BSM Models in PYTHIA 8, Eur. Phys. J. C 72 (2012) 2238 [arXiv:1109.5852] [INSPIRE].

[233] T. Sjöstrand et al., An Introduction to PYTHIA 8.2, Comput. Phys. Commun. 191 (2015) 159 [arXiv: 1410.3012] [INSPIRE].

[234] M. Cacciari, G.P. Salam and G. Soyez, The anti-kt jet clustering algorithm, JHEP 04 (2008) 063 [arXiv: 0802.1189] [InSPIRE].

[235] M. Cacciari, G.P. Salam and G. Soyez, FastJet User Manual, Eur. Phys. J. C 72 (2012) 1896 [arXiv:1111.6097] [INSPIRE].

[236] CMS collaboration, Search for supersymmetry in multijet events with missing transverse momentum in proton-proton collisions at 13 TeV, Phys. Rev. D 96 (2017) 032003 [arXiv: 1704.07781] [INSPIRE].

[237] A. Denner, S. Dittmaier, M. Roth and D. Wackeroth, Predictions for all processes $e^{+} e^{-} \rightarrow$ 4 fermions $+\gamma$, Nucl. Phys. B 560 (1999) 33 [hep-ph/9904472] [INSPIRE].

[238] A. Denner, S. Dittmaier, M. Roth and L.H. Wieders, Electroweak corrections to charged-current $e^{+} e^{-} \rightarrow 4$ fermion processes: Technical details and further results, Nucl. Phys. B 724 (2005) 247 [Erratum ibid. B 854 (2012) 504] [hep-ph/0505042] [INSPIRE]. 